Resolubilidade perto do conjunto característico para uma classe de operadores diferenciais parciais de primeira ordem

Wanderley Aparecido Cerniauskas 



\section{Resolubilidade perto do conjunto característico para uma classe de operadores diferenciais parciais de primeira ordem}

\section{Wanderley Aparecido Cerniauskas}

Orientador: Prof. Dr. Paulo Leandro Dattori da Silva Coorientador: Prof. Dr. Adalberto Panobianco Bergamasco

Tese apresentada ao Instituto de Ciências Matemáticas e de Computação - ICMC-USP, como parte dos requisitos para obtenção do título de Doutor em Ciências - Matemática. VERSÃO REVISADA 
Ficha catalográfica elaborada pela Biblioteca Prof. Achille Bassi e Seção Técnica de Informática, ICMC/USP, com os dados fornecidos pelo(a) autor(a)

\begin{tabular}{|c|c|}
\hline \multirow[t]{3}{*}{ C411r } & $\begin{array}{l}\text { Cerniauskas, Wanderley Aparecido } \\
\quad \text { Resolubilidade perto do conjunto caraterístico } \\
\text { para uma classe de operadores diferenciais parciais } \\
\text { de primeira ordem / Wanderley Aparecido } \\
\text { Cerniauskas; orientador Paulo Leandro Dattori da } \\
\text { Silva; co-orientador Adalberto Panobianco } \\
\text { Bergamasco. -- São Carlos, } 2014 \text {. } \\
\quad 76 \text { p. }\end{array}$ \\
\hline & $\begin{array}{l}\text { Tese (Doutorado - Programa de Pós-Graduação em } \\
\text { Matemática)-- Instituto de Ciências Matemáticas e } \\
\text { de Computação, Universidade de São Paulo, } 2014 \text {. }\end{array}$ \\
\hline & $\begin{array}{l}\text { 1. Resolubilidade semi-global. 2. Condição (P). } \\
\text { 3. Campos Vetoriais complexos. I. Silva, Paulo } \\
\text { Leandro Dattori da, orient. II. Bergamasco, } \\
\text { Adalberto Panobianco, co-orient. III. Título. }\end{array}$ \\
\hline
\end{tabular}


Dedico esta tese aos meus pais

André e Maria, por me oferecerem condições para eu chegar até aqui, por todo amor e carinho. 



\section{Agradecimentos}

Ao meu orientador Paulo Leandro Dattori da Silva pela orientação e pelo empenho dedicado.

Ao meu coorientador Adalberto Panobianco Bergamasco pela dedicação, apoio e incentivo.

À minha esposa Ana Sofia e à minha enteada Juliana Zuanon pelo carinho e pelos momentos de alegria.

Aos meus irmãos Renato e Val, aos meus sobrinhos Bruno, Kauan e Laura, enfim a toda minha familia.

À Universidade Estadual de Ponta Grossa, incluindo os amigos e funcionários do DEMAT que permitiram minha liberação para o Doutorado.

Aos professores do ICMC que contribuíram para minha formação acadêmica.

Aos funcionários do ICMC pelo profissionalismo.

Ao mestre Takamori pelos valiosos ensinamentos.

A todos os amigos que conquistei em São Carlos, em especial a Adriana Ramos e a Juliana Pereira.

A todos aqueles que contribuíram de forma direta ou indireta ao desenvolvimento deste trabalho. 

Seja

$$
L=\partial / \partial t+(a(x)+i b(x)) \partial / \partial x, b \not \equiv 0,
$$

um campo vetorial complexo definido em $A_{\epsilon}=(-\epsilon, \epsilon) \times S^{1}, \epsilon>0$, sendo $a, b \in$ $C^{\infty}((-\epsilon, \epsilon) ; \mathbb{R})$ e $(x, t) \in(-\epsilon, \epsilon) \times S^{1}$.

Assuma que $b^{-1}(0)=\{0\}$. Este trabalho trata da resolubilidade perto do conjunto característico $\{0\} \times S^{1}$ da equação

$$
L u=p u+f, \quad p, f \in C^{\infty}\left(A_{\epsilon}\right) .
$$

A relação entre as ordens de anulamento das funções $a$ e $b$ em $x=0$ e certas médias da função $p$ tem influência na resolubilidade. 



\section{Abstract}

Let

$$
L=\partial / \partial t+(a(x)+i b(x)) \partial / \partial x, b \not \equiv 0
$$

be a complex vector field defined in $A_{\epsilon}=(-\epsilon, \epsilon) \times S^{1}, \epsilon>0$, where $a, b \in C^{\infty}((-\epsilon, \epsilon) ; \mathbb{R})$ and $(x, t) \in(-\epsilon, \epsilon) \times S^{1}$.

Assume that $b^{-1}(0)=\{0\}$. This work deals with the solvability near the characteristic set $\{0\} \times S^{1}$ of the equation

$$
L u=p u+f, \quad p, f \in C^{\infty}\left(A_{\epsilon}\right) .
$$

The interplay between the orders of vanishing of the functions $a$ and $b$ at $x=0$ and certain averages of the function $p$ has influence in the solvability. 



\section{Sumário}

Introdução

1 Preliminares 3

2 Equação Homogênea $\quad 7$

3 Equação não homogênea 20

3.1 Existência de solução . . . . . . . . . . . . . . . . . . 21

3.2 Não existência de solução . . . . . . . . . . . . . . . . . . . . . . 52

3.3 Resultados finais . . . . . . . . . . . . . . . . . . . . . 72 

Neste trabalho, vamos lidar com campos vetoriais complexos da forma

$$
L=\partial / \partial t+(a(x)+i b(x)) \partial / \partial x, b \not \equiv 0, \quad(x, t) \in(-\epsilon, \epsilon) \times S^{1},
$$

sendo $\epsilon>0$ e $a, b \in C^{\infty}\left((-\epsilon, \epsilon) \times S^{1} ; \mathbb{R}\right)$, para os quais o conjunto $\Sigma$ dos pontos onde $L$ deixa de ser elítico é a circunferência $\Sigma=\{0\} \times S^{1}$. Além disso, assumiremos que $L$ é de tipo infinito em $\Sigma$ (ver Definição 1.1); portanto, $L$ é elítico em $A_{\epsilon} \backslash \Sigma$ e $(a+i b)(0)=0$.

Um campo $L$ da forma $(0.1)$ satisfaz a condição $(\mathcal{P})$ se, e somente se a função $b(x)$ não muda de sinal em nenhuma curva integral do campo vetorial real $\partial / \partial t+a(x) \partial / \partial x$ (ver Definição 1.3 e Teorema 1.4). Portanto, $L$ satisfaz a condição $(\mathcal{P})$ já que $a(0)=0$.

Seja $A_{\epsilon} \doteq(-\epsilon, \epsilon) \times S^{1}, \epsilon>0$, e seja $p \in C^{\infty}\left(A_{\epsilon}\right)$ uma função fixada. Nosso objetivo é estudar a resolubilidade semi-global do operador $\mathcal{L}_{p}: C^{\infty}\left(A_{\epsilon}\right) \rightarrow C^{\infty}\left(A_{\epsilon}\right)$ definido por

$$
\mathcal{L}_{p} u \doteq L u-p u
$$

sendo $L$ o campo dado por (0.1).

A resolubilidade local é bem entendida; a condição $(\mathcal{P})$ caracteriza a resolubilidade local e devido a resultados de Hörmander as soluções locais da equação

$$
\mathcal{L}_{p} u=f, \quad f \in C^{\infty}\left(A_{\epsilon}\right)
$$

podem ser obtidas em $C^{\infty}$.

O problema é ainda relevante se considerarmos a resolubilidade em uma vizinhança "cheia" de $\Sigma$. Mais precisamente, se considerarmos a resolubilidade no seguinte sentido. 
Dizemos que $\mathcal{L}_{p}$ é resolúvel em $\Sigma$ se existe um subespaço $\mathcal{F} \subset C^{\infty}\left(A_{\epsilon}\right)$ de codimensão finita tal que para cada $f \in \mathcal{F}$ existe $u \in C^{\infty}\left(A_{\epsilon}\right)$ solução da equação $\mathcal{L}_{p} u=f$ em uma vizinhança de $\Sigma$.

Podemos assumir que

- $(a+i b)(x)=x^{n} a_{0}(x)+i x^{m} b_{0}(x), n, m \in \mathbb{Z}_{+}$para todo $x \in(-\epsilon, \epsilon)$;

- se a função $a$ não é flat em $x=0$ então $n$ é a ordem de anulamento de $a$ em $x=0$; se a função $a$ é flat em $x=0$ então $n \geq m$.

Vamos analisar a resolubilidade de $\mathcal{L}_{p}$ em $\Sigma$ sob a hipótese $2 \leq m<2 n-1$; em particular mostraremos que se $m-n>0$ então a relação entre a diferença $m-n$ e certas médias da função $p$ tem influência na resolubilidade de $\mathcal{L}_{p}$.

O Capítulo 1 contém resultados que auxiliam na compreensão do texto. Apresentamos alguns resultados sobre resolubilidade que estão relacionados com o campo $L$ dado por $(0.1)$.

No Capítulo 2 lidamos com a equação homogênea $\mathcal{L}_{p} u=0$. Encontramos condições necessárias e suficientes sobre $p$ para que a equação $\mathcal{L}_{p} u=0$ tenha solução de classe $C^{\infty}$, em uma vizinhança de $\Sigma$, com ordem de anulamento finita em $\Sigma$, uniforme em t. Também apresentamos condições sobre $p$, sob as quais é possível obter solução não trivial, de classe $C^{\infty}$ e flat em $\Sigma$.

No Capítulo 3 lidamos com a existência de solução; apresentamos condições sobre $p$, sob as quais é possível determinar condições necessárias e suficientes sobre $f$ para que a equação $\mathcal{L}_{p} u=f$ tenha solução de classe $C^{\infty}$, em uma vizinhança de $\Sigma$. 


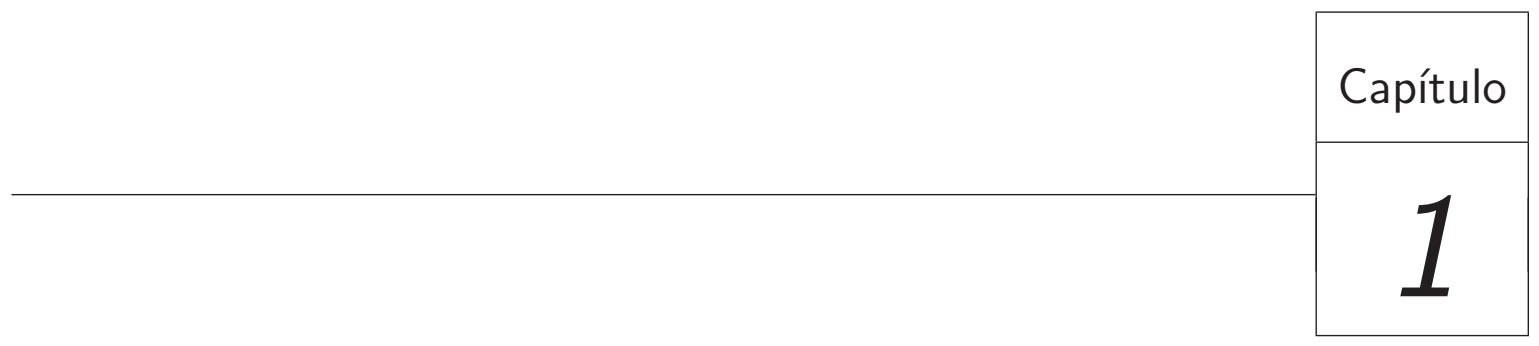

Preliminares

Este capítulo contém resultados que auxiliam na compreensão do texto.

Seja $L$ um campo vetorial sobre uma variedade suave $\Omega$, de dimensão $\eta$. Denote por $\rho(x, \xi)$ o símbolo principal de $L$; localmente podemos escrever $\rho(x, \xi)=\sum_{j=1}^{\eta} d_{j}(x) \xi_{j}$.

Definição 1.1 (Campo vetorial de tipo finito e infinito). Sejam $C^{1}=[L, \bar{L}], C^{2}=$ $\left[L, C^{1}\right], \ldots, C^{n+1}=\left[L, C^{n}\right]$, sendo $[$,$] o colchete de Lie. Dizemos que o campo vetorial$ $L$ é de tipo finito $\nu$ em $p \in \Sigma$, se $L$ e $C^{j}$ são linearmente dependentes para cada $j<\nu$, mas $L$ e $C^{\nu}$ são linearmente independentes. Se $L$ não é de tipo finito em $p$ então $L$ é de tipo infinito em $p$.

Definição 1.2. Seja $\varphi$ uma função suave a valores complexos sobre $T^{*} \Omega \backslash$ O. Uma curva bi-característica de $\Re(\varphi)$ é uma curva integral do campo Hamiltoniano de $\Re(\varphi)$, sobre a qual $\Re(\varphi)$ se anula.

Definição 1.3. Diz-se que $L$ satisfaz a condição $(\mathcal{P})$, em um aberto $V \subset \Omega$, se não existe função suave a valores complexos, positivamente homogênea, $q(x, \xi)$, sobre $T^{*} V \backslash \mathrm{O}$, tal que $\Im(q \rho)$ assume tanto valores positivos quanto negativos sobre uma curva bicaracterística de $\Re(q \rho)$, em $V$, sobre a qual $q \neq 0$.

Ver [7], Definição 3.1. 
Teorema 1.4. Cada uma das seguintes condições é necessaria e suficiente para que $L$ satisfaça a condição $(\mathcal{P})$ em $V$ :

(a) Não existe função $C^{\infty}$ a valores complexos $q$ em $T^{*} V \backslash \mathrm{O}$, tal que $\Im(q \rho)$ assume tanto valores positivos quanto negativos sobre uma curva bi-característica de $\Re(q \rho)$, em $V$, sobre o qual $q \neq 0$.

(b) Se $\Gamma$ é um ponto característico ou uma curva bi-característica com projeção regular injetiva em $S^{*}(V)$, então existe uma função $q$ de classe $C^{\infty}$, numa vizinhança $W$ de $\Gamma$, tal que $\Re\left(H_{q \rho}\right) \neq 0$ em $W$ e $\Im(q \rho)$ não muda de sinal sobre qualquer bicaracterística de $\Re(q \rho)$ em $W$.

Ver [7], Teorema 3.7 .

Definição 1.5. Definimos o conjunto característico da estrutura associada a $\mathbf{L}$ como sendo

$$
\mathcal{C}(L)=\left\{(x, t) \in A_{\epsilon} ; L_{(x, t)} \text { e } \bar{L}_{(x, t)} \text { são linearmente independentes }\right\}
$$

Suponha que $L$ é dado por

$$
L=\partial / \partial t+(a(x)+i b(x)) \partial / \partial x, b \not \equiv 0, \quad(x, t) \in \Omega
$$

e que $\mathcal{C}(L)=\{0\} \times S^{1}$, sendo $\Omega=(-\epsilon, \epsilon) \times S^{1}, \epsilon>0$ e $a, b \in C^{\infty}(\Omega ; \mathbb{R})$. Então, pelo Teorema $1.4, L$ satisfaz a condição $(\mathrm{P})$ se, e somente se, $b(\gamma(s))$ não muda de sinal, para qualquer $\gamma$ curva integral de $\Re(L)$; a curva integral de $\Re(L)$, passando por $(x, t)$, tem a forma $\gamma_{(x, t)}(s)=\phi(x, s)$, sendo $\partial_{s} \phi(x, s)=a(\phi(x, s))$.

Se $L$, dado por (1.1), é resolúvel em $\Sigma=\{0\} \times S^{1}$ então necessariamente a condição $(\mathcal{P})$ deve ser satisfeita numa vizinhança aberta de $\Sigma$ ([9], Corolário 26.4.8). Além disso, se

(CG) Qualquer ponto característico de $L$ sobre $K$ pertence a um intervalo compacto de curva bicaracterística de $\Re(q \rho)$, sobre a qual $q \neq 0$, com pontos inicial e final não característicos sobre $\Sigma$.

então a condição $(\mathcal{P})$ implica resolubilidade de $L$ em $\Sigma$ num sentido mais forte: existem soluções em $C^{\infty}(\Omega)$ ([9], Teorema 26.11.3). Assim, supondo a condição (CG) satisfeita, é possível obter uma recíproca do Corolário 26.4 .8 em [9].

Hörmander (veja [9]) denomina o operadore $L$, definido acima, que satisfaz a condição $(\mathcal{P})$ e a condição geométrica $(\mathrm{CG})$ como operadores de tipo principal. 
Note que o operador $L$, dado por (1.1), não satisfaz a condição (CG) já que ele é tangente a $\Sigma$; consequentemente $L$ não é de tipo principal.

Segue de [2], [6] e [5], os seguintes resultados:

Teorema 1.6. Seja $L$ dado por (1.1). Assuma que $(a+i b)(x)=x^{n} a_{0}(x)+i x^{m} b_{0}(x) e$ seja $r=\min \{m, n\}$. Se $b(0) \neq 0$ e $2 \leqslant m<2 n-1$, então dada $f \in C^{\infty}\left(A_{\epsilon}\right)$, a equação $L u=f$ tem solução $u \in C^{\infty}\left(A_{\epsilon}\right)$, em uma vizinhança de $\Sigma$, se, e somente se, $f$ satisfaz

$$
\int_{0}^{2 \pi} \frac{\partial^{j} f}{\partial x^{j}}(0, t) d t=0, j=0, \ldots, r-1
$$

Teorema 1.7. Seja L dado por (1.1) e seja $r=\min \{m, n\}$. Se $(a+i b)(x)=x^{n} a_{0}(x)+$ $i x^{m} b_{0}(x)$ e é satisfeita uma das seguintes condições

$$
\begin{aligned}
& 1 \star . \quad a_{0}(0) \neq 0 \text { e } m>2 n-1 ; \\
& 2 \star . \quad b_{0}(0) \neq 0 \text { e } m=1 ; \\
& 3 \star . \quad a_{0}(0) \neq 0, b_{0}(0) \neq 0, m=2 n-1 \text { e } n \geqslant 2 ;
\end{aligned}
$$

então existe uma função $f \in C^{\infty}\left(A_{\epsilon}\right)$ satisfazendo

$$
\int_{0}^{2 \pi} \frac{\partial^{j} f}{\partial x^{j}}(0, t) d t=0, j=0, \ldots, r-1
$$

para o qual a equação $L u=f$ não tem solução de classe $C^{\infty}$ em nenhuma vizinhança de $\Sigma$.

Combinando os Teoremas 1.6 e 1.7 obtemos o seguinte resultado sobre a equação $L u=f$.

Teorema 1.8. Seja $L$ dado por (1.1). Assuma que $(a+i b)(x)=x^{n} a_{0}(x)+i x^{m} b_{0}(x)$ e $a_{0}(0)+i b_{0}(0) \neq 0$. Seja $r=\min \{m, n\}$ e seja $\mathcal{F}$ o subespaço de $C^{\infty}\left(A_{\epsilon}\right)$ de todas funções $f$ satisfazendo

$$
\int_{0}^{2 \pi} \frac{\partial^{j} f}{\partial x^{j}}(0, t) d t=0, j=0, \ldots, r-1
$$

Então, para cada $f \in \mathcal{F}$, a equação Lu $=f$ tem uma solução de classe $C^{\infty}$, em uma vizinhança de $\Sigma$ se, e somente se, $2 \leq m<2 n-1$.

Em particular, para $L$ dado por (1.1), se $(a+i b)(x)=x^{n} a_{0}(x)+i x^{m} b_{0}(x)$, $a_{0}(0)+i b_{0}(0) \neq 0$ e $2 \leqslant m<2 n-1$ então $L$ é resolúvel em $\Sigma$. 
Assuma que $L$ é dado por (1.1), $(a+i b)(x)=x^{n} a_{0}(x)+i x^{m} b_{0}(x), 2 \leq m \leq n$ e que $b_{0}(0) \neq 0$. Em [10] foi provado que existe um único polinômio $P(x)$ de grau no máximo $m-1$ com $\Re P(0)<0$ e um único $\mu \in \mathbb{C}$ tal que $L$, dado por $(0.1)$, é equivalente ao campo vetorial

$$
R_{m-1}=\frac{\partial}{\partial t}-i \frac{x^{m}}{x P^{\prime}(x)-(m-1) P(x)+\mu x^{m-1}} \frac{\partial}{\partial x} .
$$

Logo, o estudo da equação $L u=p u+f$, em uma vizinhança de $\Sigma$, é reduzido à correspondente equação para o campo vetorial $R_{m-1}$, em uma vizinhança do círculo $\Sigma$.

O artigo [4] lida com a resolubilidade da equação $R_{m-1} u=p u+f$. Logo, a resolubilidade de (1.1) é bem entendida quando $2 \leqslant m \leqslant n$. Contudo, a resolubilidade de (1.1), quando $n<m$ é ainda um problema em aberto. 


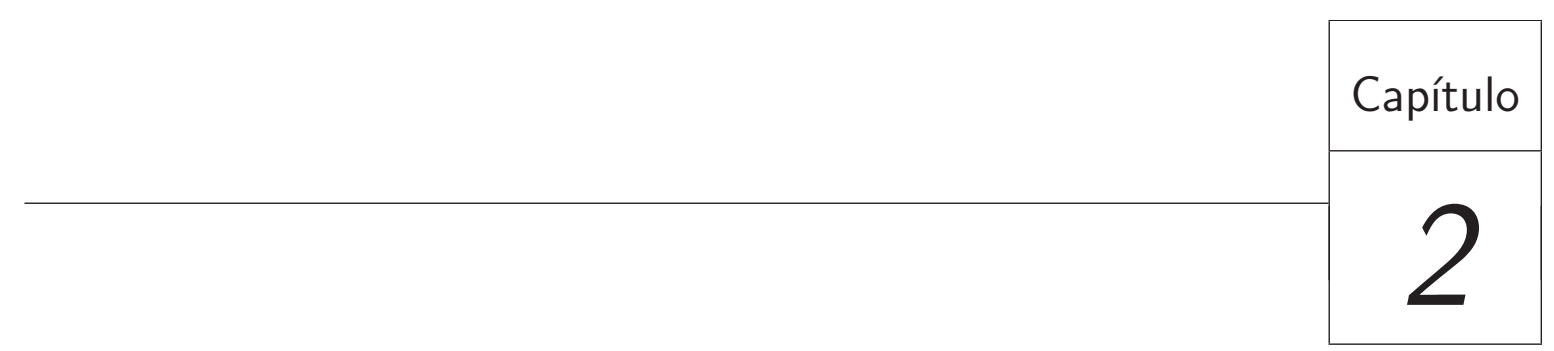

\section{Equação Homogênea}

Seja

$$
L=\partial / \partial t+\left(x^{n} a_{0}(x)+i x^{m} b_{0}(x)\right) \partial / \partial x, b_{0} \not \equiv 0, \quad(x, t) \in(-\epsilon, \epsilon) \times S^{1},
$$

um campo vetorial complexo definido em $A_{\epsilon}=(-\epsilon, \epsilon) \times S^{1}$, sendo $a_{0}, b_{0} \in C^{\infty}\left(A_{\epsilon} ; \mathbb{R}\right)$ e $m, n \in \mathbb{Z}_{+}$. Assumimos que

- $2 \leq m<2 n-1$

- $b_{0}(0)>0$;

- se a função $a:(-\epsilon, \epsilon) \mapsto \mathbb{R}$, dada por $a(x)=x^{n} a_{0}(x)$, não é flat em $x=0$ então $n$ é a ordem de anulamento de $a$ em $x=0$; se a função $a$ é flat em $x=0$ então $n \geq m$.

A hipótese $b_{0}(0)>0$ não é restritiva. De fato se $b_{0}(0)<0$, fazendo a mudança $\bar{t}=-t$, $x=x$, obtemos que $L$ nas coordenadas $(x, \bar{t})$ tem a forma

$$
-\partial / \partial \bar{t}+(a+i b)(x) \partial / \partial x
$$


sendo $b:(-\epsilon, \epsilon) \mapsto \mathbb{R}$, a função dada por $b(x)=x^{m} b_{0}(x)$; consequentemente, podemos considerar $L$ dado por

$$
\partial / \partial \bar{t}-(a+i b)(x) \partial / \partial x .
$$

Seja $p \in C^{\infty}\left(A_{\epsilon}\right)$.

Defina o operador $\mathcal{L}_{p}: C^{\infty}\left(A_{\epsilon}\right) \rightarrow C^{\infty}\left(A_{\epsilon}\right)$ por

$$
\mathcal{L}_{p} u \doteq L u-p u
$$

Neste capítulo vamos procurar por soluções $C^{\infty}$ não triviais, em uma vizinhança de $\Sigma$, da equação homogênea

$$
\mathcal{L}_{p} u=0
$$

definida em $A_{\epsilon}$, sendo $L$ dado por (2.1).

Proposição 2.1. A equação (2.3) tem uma solução $u \in C^{\infty}\left(A_{\epsilon}\right)$, com $u\left(0, t_{0}\right) \neq 0$ para algum $t_{0} \in S^{1}$ se, e somente se, a função p satisfaz

$$
\frac{1}{2 \pi i} \int_{0}^{2 \pi} p(0, t) d t \in \mathbb{Z}
$$

$e$

$$
\int_{0}^{2 \pi} \frac{\partial^{j} p}{\partial x^{j}}(0, t) d t=0, j=1, \ldots, r-1,
$$

sendo $r=\min \{m, n\}$.

Demonstração. Suponha que exista $u \in C^{\infty}\left(A_{\epsilon}\right)$, com $u\left(0, t_{0}\right) \neq 0$ para algum $t_{0} \in S^{1}$, solução de $L u=p u$ em uma vizinhança de $\Sigma$. Então, $u_{0}(t) \doteq u(0, t)$ é a solução do problema de Cauchy:

$$
\left\{\begin{array}{l}
v^{\prime}(t)=p(0, t) v(t) \\
v\left(t_{0}\right)=u\left(0, t_{0}\right)
\end{array} .\right.
$$

Portanto, temos $u(0, t)=c \cdot e^{\int_{0}^{t} p(0, \sigma) d \sigma}$, sendo $c=\frac{u\left(0, t_{0}\right)}{e^{\int_{0}^{t_{0}} p(0, \sigma) d \sigma}} \neq 0$. Como $u$ é $2 \pi$ periódica na variável $t$, concluímos que (2.4) é satisfeito.

Defina

$$
w(x, t) \doteq \bar{c} u(x, t) e^{-\int_{0}^{t} p(0, \sigma) d \sigma} .
$$

É fácil ver que $w(x, t)$ é uma solução da equação

$$
L w=\widetilde{p} w
$$


sendo $\tilde{p}(x, t)=p(x, t)-p(0, t)$. Além disso, note que $w(0, t)=|c|^{2}>0$ para todo $t \in S^{1}$; assumindo $\epsilon$ suficientemente pequeno, temos $\Re w(x, t)>0$ para todo $(x, t) \in A_{\epsilon}$ (em particular $w(x, t) \neq 0$ para todo $\left.(x, t) \in A_{\epsilon}\right)$; então, a função $v \in C^{\infty}\left(A_{\epsilon}\right)$, dada por

$$
e^{v}=w
$$

é bem definida.

Logo, de (2.6) temos $\widetilde{p} w=L e^{v}=e^{v} L v=w L v$ e, consequentemente, $v$ é uma solução $C^{\infty}$ de

$$
L v=\widetilde{p},
$$

em uma vizinhança de $\Sigma$.

Daí,

$$
\int_{0}^{2 \pi} \frac{\partial^{j} \widetilde{p}}{\partial x^{j}}(0, t) d t=0, \quad j=0, \ldots, r-1
$$

Como $L v$ se anula de ordem $r$ em $\{x=0\}, \widetilde{p}$ satisfaz

$$
\int_{0}^{2 \pi} \frac{\partial^{j}}{\partial x^{j}}\left[(a+i b) \frac{\partial v}{\partial x}\right](0, t) d t=0, \quad j=0, \ldots, r-1
$$

consequentemente, $p$ satisfaz (2.5).

Reciprocamente, suponha que $p$ satisfaz (2.4) e (2.5). Então:

- a função $t \mapsto e^{\int_{0}^{t} p(0, \sigma) d \sigma}$ é $2 \pi$-periódica;

- a função $\widetilde{p}$, definida por $\widetilde{p}(x, t) \doteq p(x, t)-p(0, t)$ em $A_{\epsilon}$, satisfaz

$$
\int_{0}^{2 \pi} \frac{\partial^{j} \widetilde{p}}{\partial x^{j}}(0, t) d t=0, \quad j=0, \ldots, r-1
$$

consequentemente, pelo Teorema 1.8 , existe $\varphi \in C^{\infty}\left(A_{\epsilon}\right)$ solução de $L \varphi=\tilde{p}$ em uma vizinhança de $\Sigma$.

Portanto, a função $u \in C^{\infty}\left(A_{\epsilon}\right)$ definida por

$$
u(x, t)=e^{\varphi(x, t)+\int_{0}^{t} p(0, \sigma) d \sigma}
$$

é uma solução de $(2.3)$ em uma vizinhança de $\Sigma$; claramente, $u(x, t) \neq 0$ para todo $(x, t) \in A_{\epsilon}$.

Agora, fixamos as notações: 
- $r=\min \{m, n\} \geqslant 2$;

- $\alpha_{0} \doteq\left[\left(x^{n-r} a_{0}(x)+i x^{m-r} b_{0}(x)\right]_{x=0}\right.$.

Para cada $\ell \in \mathbb{Z}_{+}$defina

$$
q_{\ell}(x, t) \doteq p(x, t)-\ell\left[x^{n-1} a_{0}(x)+i x^{m-1} b_{0}(x)\right] .
$$

Note que

$$
\frac{\partial^{j} q_{\ell}}{\partial x^{j}}(0, t)=\frac{\partial^{j} p}{\partial x^{j}}(0, t), \quad \text { se } \quad j=0, \ldots, r-2 .
$$

Teorema 2.2. Seja $\ell \in\{0,1, \ldots\}$. Existe $u \in C^{\infty}\left(A_{\epsilon}\right)$, com ordem de anulamento $\ell$ em $\Sigma$ uniforme em $t$, solução de (2.3) em uma vizinhança de $\Sigma$ se, e somente se, p satisfaz

$$
\begin{aligned}
& \frac{1}{2 \pi i} \int_{0}^{2 \pi} p(0, t) d t \in \mathbb{Z}, \\
& \int_{0}^{2 \pi} \frac{\partial^{j} p}{\partial x^{j}}(0, t) d t=0, j=1, \ldots, r-2,
\end{aligned}
$$

$e$

$$
\int_{0}^{2 \pi} \frac{\partial^{r-1} p}{\partial x^{r-1}}(0, t) d t=2 \pi \ell \alpha_{0}(r-1) !
$$

Demonstração. O caso $\ell=0$ segue da Proposição 2.1. Podemos, então, considerar $\ell \geqslant 1$. Seja $w \in C^{\infty}\left(A_{\epsilon}\right)$, com $w \not \equiv 0$ em $\Sigma$. Assuma que $u(x, t)=x^{\ell} w(x, t)$ é solução $C^{\infty}$ de (2.3) em uma vizinhança de $\Sigma$. Então,

$$
x^{\ell} p w=p u=L u=x^{\ell} L w+w L x^{\ell}=x^{\ell} L w+w \ell x^{\ell-1}\left(x^{n} a_{0}+i x^{m} b_{0}\right),
$$

ou seja,

$$
x^{\ell} L w+x^{\ell} \ell\left(x^{n-1} a_{0}+i x^{m-1} b_{0}\right) w=x^{\ell} p w
$$

consequentemente,

$$
L w+\ell\left(x^{n-1} a_{0}+i x^{m-1} b_{0}\right) w=p w
$$

para $x \neq 0$, e, por continuidade, para $x=0$.

Logo, $w$ é uma solução $C^{\infty}$ de $L w=q_{\ell} w$, em uma vizinhança de $\Sigma$; além disso, $w \not \equiv 0$ em $\Sigma$. Portanto, pela Proposição 2.1, $q_{\ell}$ satisfaz

$$
\frac{1}{2 \pi i} \int_{0}^{2 \pi} q_{\ell}(0, t) d t \in \mathbb{Z}
$$


e

$$
\int_{0}^{2 \pi} \frac{\partial^{j} q_{\ell}}{\partial x^{j}}(0, t) d t=0, j=1, \ldots, r-1
$$

Agora, de $q_{\ell}(0, t)=p(0, t)$ concluímos que $p$ satisfaz (2.11). Além disso, de (2.10) obtemos

$$
\int_{0}^{2 \pi} \frac{\partial^{j} p}{\partial x^{j}}(0, t) d t=0, j=1, \ldots, r-2
$$

portanto, (2.12) é satisfeita.

Finalmente, para $j=r-1$, temos

$$
\begin{aligned}
0=\int_{0}^{2 \pi} \frac{\partial^{r-1} q_{\ell}}{\partial x^{r-1}}(0, t) d t & =\int_{0}^{2 \pi} \frac{\partial^{r-1} p}{\partial x^{r-1}}(0, t) d t-\int_{0}^{2 \pi} \ell \alpha_{0}(r-1) ! d t \\
& =\int_{0}^{2 \pi} \frac{\partial^{r-1} p}{\partial x^{r-1}}(0, t) d t-2 \pi \ell \alpha_{0}(r-1) !
\end{aligned}
$$

Portanto, (2.13) é satisfeita.

Reciprocamente, assuma que (2.11), (2.12) e (2.13) são satisfeitas.

Então, a função $q_{\ell}$ satisfaz

$$
\frac{1}{2 \pi i} \int_{0}^{2 \pi} q_{\ell}(0, t) d t \in \mathbb{Z}
$$

e

$$
\int_{0}^{2 \pi} \frac{\partial^{j} q_{\ell}}{\partial x^{j}}(0, t) d t=0, j=1, \ldots, r-1 .
$$

Logo, pela Proposição 2.1, a equação $L w=q_{\ell} w$ tem uma solução $w \in C^{\infty}\left(\Omega_{\epsilon}\right)$, com $w(0, t) \not \equiv 0$.

Portanto, $u(x, t)=x^{\ell} w(x, t)$ é uma solução $C^{\infty}$ de $L u=p u$ em uma vizinhança de $\Sigma$.

Para cumprirmos o objetivo deste capítulo, resta-nos assumir que (2.4) ou (2.5) falha e, além disso, considerar que

$$
\int_{0}^{2 \pi} \frac{\partial^{r-1} p}{\partial x^{r-1}}(0, t) d t \neq 2 \pi \ell \alpha_{0}(r-1) !, \quad \forall \ell \in \mathbb{Z}_{+},
$$

quando (2.11) e (2.12) são satisfeitas. Sob estas hipóteses, pelo Teorema 2.2, se a equação (2.3) tem uma solução $C^{\infty}$ então esta solução deve ser flat em $\Sigma$. 
Agora, para cada $j \geq 0$, fixamos a notação

$$
\lambda_{j}=\frac{1}{2 \pi} \int_{0}^{2 \pi} p_{j}(t) d t
$$

sendo

$$
p_{j}(t)=\frac{1}{j !} \frac{\partial^{j} p}{\partial x^{j}}(0, t)
$$

Note que (2.4) é equivalente a $\lambda_{0} \in i \mathbb{Z}$; também, (2.5) é equivalente a $\lambda_{j}=0$ para cada $j=0, \ldots, r-1$.

Vamos procurar por solução flat não trivial de $\mathcal{L}_{p} u=0$.

Da demonstração da Proposição 3.4 (ver página 40) podemos afirmar que, para encontrar uma solução $u$ flat (não trivial) de $\mathcal{L}_{p} u=0$, é suficiente encontrar uma solução flat não trivial da equação

$$
L u=\left(\sum_{j=0}^{r-1} \lambda_{j} x^{j}\right) u
$$

Para cada $j=0, \ldots, r-1$, defina

$$
C_{j+}(x) \doteq-\int_{x}^{\epsilon} \frac{y^{j}}{a+i b}(y) \mathrm{d} y, \quad \text { para } \quad x>0
$$

e

$$
C_{j-}(x) \doteq \int_{-\epsilon}^{x} \frac{y^{j}}{a+i b}(y) \mathrm{d} y, \quad \text { para } \quad x<0
$$

Proposição 2.3. Assuma que (2.4) ou (2.5) falha. Defina o inteiro $j_{0}$ como segue:

(i) se $\Re\left(\lambda_{j}\right)=0$ para cada $j \in\{0, \cdots, r-1\}$, seja $j_{0}=r-1$;

(ii) se $\Re\left(\lambda_{j}\right) \neq 0$ para algum $j \in\{0, \cdots, r-1\}$, seja $j_{0}=\min _{0 \leqslant j \leqslant r-1}\left\{j: \Re\left(\lambda_{j}\right) \neq 0\right\}$.

Suponha que $m-n \leq j_{0}$. Então, a equação (2.15) tem uma solução u não trivial, $C^{\infty}$ e flat em $\Sigma$. Além disso, $u(x, t) \neq 0$ se $(x, t) \in A_{\epsilon} \backslash \Sigma$.

Demonstração. Vamos procurar por solução não trivial de (2.15) na forma

$$
u(x, t)=\left\{\begin{array}{l}
w(x) e^{i \kappa^{+} t}, x \geqslant 0 \\
w(x) e^{i \kappa^{-} t}, x<0
\end{array}\right.
$$

sendo $\kappa^{+}, \kappa^{-} \in \mathbb{Z}, w \in C^{\infty}((-\epsilon, \epsilon)), w(x) \neq 0$ para $x \neq 0$, e $w$ flat em $x=0$. 
Para $u$ dado por (2.16) a equação (2.15) fornece

$$
\left[x^{n} a_{0}(x)+i x^{m} b_{0}(x)\right] w^{\prime}(x)=\left(-i \kappa^{ \pm}+\sum_{j=0}^{r-1} \lambda_{j} x^{j}\right) w(x) \quad \text { em } \quad I_{\epsilon}^{ \pm},
$$

sendo $I_{\epsilon}^{+}=(0, \epsilon)$ e $I_{\epsilon}^{-}=(-\epsilon, 0)$.

Simples cálculos mostram que

$$
w_{ \pm}(x)=e^{-i \kappa^{ \pm} C_{0 \pm}(x)+\sum_{j=0}^{r-1} \lambda_{j} C_{j \pm}(x)}
$$

é uma solução de (2.17) em $I_{\epsilon}^{ \pm}$.

Defina

$$
w(x)=\left\{\begin{array}{rrr}
e^{-i \kappa^{+} C_{0+}(x)+\sum_{j=0}^{r-1} \lambda_{j} C_{j+}(x)}, & \text { se } & 0<x<\epsilon \\
0, & \text { se } & x=0 \\
e^{-i \kappa^{-} C_{0-}(x)+\sum_{j=0}^{r-1} \lambda_{j} C_{j-}(x)}, & \text { se } & -\epsilon<x<0
\end{array} .\right.
$$

Mostraremos que $w$ é flat em $x=0$; consequentemente, $w \in C^{\infty}((-\epsilon, \epsilon))$.

Note que, para cada $j=0, \ldots, r-1$, temos

$$
\lambda_{j} C_{j \pm}(x)=-\int_{x}^{ \pm \epsilon} y^{j} \frac{\Re\left(\lambda_{j}\right) a+\Im\left(\lambda_{j}\right) b}{a^{2}+b^{2}}(y) \mathrm{d} y+i \int_{x}^{ \pm \epsilon} y^{j} \frac{\Re\left(\lambda_{j}\right) b-\Im\left(\lambda_{j}\right) a}{a^{2}+b^{2}}(y) d y
$$

e, portanto

$$
\begin{aligned}
& \Re\left(-i \kappa^{ \pm} C_{0 \pm}(x)+\sum_{j=0}^{r-1} \lambda_{j} C_{j \pm}(x)\right) \\
& =-\int_{x}^{ \pm \epsilon}\left\{\left(\sum_{j=j_{0}}^{r-1} \Re\left(\lambda_{j}\right) y^{j}\right) \frac{a}{a^{2}+b^{2}}(y)+\left[-\kappa^{ \pm}+\Im\left(\lambda_{0}\right)+\sum_{j=1}^{r-1} \Im\left(\lambda_{j}\right) y^{j}\right] \frac{b}{a^{2}+b^{2}}(y)\right\} \mathrm{d} y \\
& =-\int_{x}^{ \pm \epsilon} \frac{1}{y^{\ell}}\left[\left(\sum_{j=j_{0}}^{r-1} \Re\left(\lambda_{j}\right) y^{j-m+n}\right) \frac{a_{0}}{b_{0}}(y)-\kappa^{ \pm}+\Im\left(\lambda_{0}\right)+\sum_{j=1}^{r-1} \Im\left(\lambda_{j}\right) y^{j}\right] y^{m+\ell} \frac{b_{0}}{a^{2}+b^{2}}(y) \mathrm{d} y,
\end{aligned}
$$

sendo

$$
\ell=\left\{\begin{array}{rl}
m, & \text { se } \quad 2 \leq m<n \\
2 n-m, & \text { se } \quad n \leq m<2 n-1
\end{array} .\right.
$$

Defina

$$
D_{\kappa^{ \pm}} \doteq \Re\left(\lambda_{j_{0}}\right)\left[x^{n-m+j_{0}} a_{0}(x)\right]_{x=0}+\left(\Im\left(\lambda_{0}\right)-\kappa^{ \pm}\right) b_{0}(0),
$$


e

$$
\begin{aligned}
g_{\kappa^{ \pm}}(x) \doteq \frac{1}{D_{\kappa^{ \pm}}}\left[\left(\sum_{j=j_{0}}^{r-1} \Re\left(\lambda_{j}\right) x^{j-m+n}\right)\right. & \frac{a_{0}}{b_{0}}(x) \\
& \left.-\kappa^{ \pm}+\Im\left(\lambda_{0}\right)+\sum_{j=1}^{r-1} \Im\left(\lambda_{j}\right) x^{j}\right] x^{m+\ell} \frac{b_{0}}{a^{2}+b^{2}}(x) .
\end{aligned}
$$

Para $\kappa^{ \pm} \in \mathbb{Z}$, tal que $\kappa^{ \pm} \neq \Im\left(\lambda_{0}\right)+\Re\left(\lambda_{j_{0}}\right)\left[x^{n-m+j_{0}} \frac{a_{0}(x)}{b_{0}(x)}\right]_{x=0}$, temos

$$
g_{\kappa^{ \pm}}(0)=\left\{\begin{aligned}
1 / b_{0}^{2}(0), & \text { se } 2 \leq m<n \\
1 /\left[a_{0}^{2}(0)+b_{0}^{2}(0)\right], & \text { se } m=n \\
1 / a_{0}^{2}(0), & \text { se } n<m<2 n-1
\end{aligned}\right.
$$

e

$$
\Re\left(-i \kappa^{ \pm} C_{0 \pm}(x)+\sum_{j=0}^{r-1} \lambda_{j} C_{j \pm}(x)\right)=-D_{\kappa^{ \pm}} \int_{x}^{ \pm \epsilon} \frac{1}{y^{\ell}} g_{\kappa^{ \pm}}(y) \mathrm{d} y .
$$

Como $g_{\kappa^{ \pm}} \in C^{\infty}((-\epsilon, \epsilon))$, assumindo $\epsilon>0$ suficientemente pequeno, podemos encontrar $\alpha, \beta>0$ tais que

$$
\alpha \leqslant g_{\kappa^{ \pm}}(x) \leqslant \beta, \quad \text { para todo } \quad x \in(-\epsilon, \epsilon) .
$$

Logo, escolhendo $\kappa^{ \pm} \in \mathbb{Z}$ tais que $D_{\kappa^{+}}>0$ e $(-1)^{\ell} D_{\kappa^{-}}<0$, obtemos:

- para $x>0$,

$$
\begin{aligned}
|w(x)|=e^{-\int_{x}^{\epsilon} \sum_{j=j_{0}}^{r-1} \Re\left(\lambda_{j}\right) y^{j} \frac{a}{a^{2}+b^{2}}(y) \mathrm{d} y-\int_{x}^{\epsilon}\left(-\kappa^{+}+\sum_{j=0}^{r-1} \Im\left(\lambda_{j}\right) y^{j}\right) \frac{b}{a^{2}+b^{2}}(y) \mathrm{d} y} \\
=e^{-D_{\kappa^{+}} \int_{x}^{\epsilon} \frac{1}{y^{\ell}} g_{\kappa^{+}}(y) \mathrm{d} y} \leqslant e^{\frac{-\left(D_{\kappa^{+}}\right) \alpha}{(\ell-1)}\left(\frac{1}{x^{\ell-1}}-\frac{1}{\epsilon^{\ell-1}}\right)}=O\left(|x|^{j}\right), \quad \forall j \in \mathbb{Z}_{+} ;
\end{aligned}
$$

- para $x<0$,

$$
\begin{aligned}
|w(x)|= & e^{\int_{-\epsilon}^{x} \sum_{j=j_{0}}^{r-1} \Re\left(\lambda_{j}\right) y^{j} \frac{a}{a^{2}+b^{2}}(y) \mathrm{d} y+\int_{-\epsilon}^{x}\left(-\kappa^{-}+\sum_{j=0}^{r-1} \Im\left(\lambda_{j}\right) y^{j}\right) \frac{b}{a^{2}+b^{2}}(y) \mathrm{d} y} \\
& =e^{(-1)^{\ell} D_{\kappa^{-}} \int_{-\epsilon}^{x} \frac{1}{(-y)^{\ell}} g_{\kappa^{-}}(y) \mathrm{d} y} \leqslant e^{\frac{(-1)^{\ell}\left(D_{\kappa^{-}}\right) \alpha}{(\ell-1)}\left(\frac{1}{|x|^{\ell-1}}-\frac{1}{\epsilon^{\ell-1}}\right)}=O\left(|x|^{j}\right), \quad \forall j \in \mathbb{Z}_{+} .
\end{aligned}
$$

Cálculos similares mostram que, para cada $\gamma \in \mathbb{Z}_{+},\left|w^{(\gamma)}(x)\right|=O\left(|x|^{j}\right), \forall j \in \mathbb{Z}_{+}$. Portanto, $w \in C^{\infty}((-\epsilon, \epsilon)), w(x) \neq 0$ se $x \neq 0$, e $w$ é flat em $x=0$; consequentemente, $u$ dado por (2.16) tem as propriedades desejadas. 
Proposição 2.4. Suponha que $\Re\left(\lambda_{j}\right) \neq 0$ para algum $j \in\{0, \cdots, r-1\}$ e, além disso, $m-n>j_{0}=\min _{0 \leq j \leq r-1}\left\{j: \Re\left(\lambda_{j}\right) \neq 0\right\}$. Temos:

(i) se $\Re\left(\lambda_{j_{0}}\right) a_{0}(0)>0$ e $n-j_{0}$ é impar, então a equação (2.15) tem uma solução $u$ de classe $C^{\infty}$, flat em $\Sigma$, tal que $u(x, t) \neq 0$ se $(x, t) \in A_{\epsilon} \backslash \Sigma$.

(ii) se $\Re\left(\lambda_{j_{0}}\right) a_{0}(0)>0$ e $n-j_{0}$ é par, então a equação (2.15) tem uma solução u de classe $C^{\infty}$, flat em $\Sigma$, tal que $u(x, t) \neq 0$ se $(x, t) \in(0, \epsilon) \times S^{1}$.

(iii) se $\Re\left(\lambda_{j_{0}}\right) a_{0}(0)<0$ e $n-j_{0}$ é par, então a equação (2.15) tem uma solução u de classe $C^{\infty}$, flat em $\Sigma$, tal que $u(x, t) \neq 0$ se $(x, t) \in(-\epsilon, 0) \times S^{1}$.

(iv) se $\Re\left(\lambda_{j_{0}}\right) a_{0}(0)<0$ e $n-j_{0}$ é ímpar, então a única solução de classe $C^{\infty}$ da equação (2.15) é $w \equiv 0$.

Demonstração. Vamos procurar por soluções não triviais de (2.15) na forma

$$
u(x, t)=w(x) e^{i k t}
$$

sendo $k \in \mathbb{Z}, w \in C^{\infty}((-\epsilon, \epsilon))$ flat em $x=0$, e $w(x) \not \equiv 0$.

Para $u$ dado por (2.24), a equação (2.15) fornece

$$
\left[x^{n} a_{0}(x)+i x^{m} b_{0}(x)\right] w^{\prime}(x)=\left(-i k+\sum_{j=0}^{n-1} \lambda_{j} x^{j}\right) w(x) \quad \text { em } \quad I_{\epsilon}^{ \pm}
$$

sendo $I_{\epsilon}^{+}=(0, \epsilon)$ e $I_{\epsilon}^{-}=(-\epsilon, 0)$.

Simples cálculos mostram que

$$
w_{ \pm}(x)=e^{-k i C_{0 \pm}(x)+\sum_{j=0}^{n-1} \lambda_{j} C_{j \pm}(x)}
$$

é uma solução de (2.25) em $I_{\epsilon}^{ \pm}$.

Sob nossas hipóteses, obtemos

$$
\begin{gathered}
\Re\left(-k i C_{0 \pm}(x)+\sum_{j=0}^{n-1} \lambda_{j} C_{j \pm}(x)\right) \\
=-\int_{x}^{ \pm \epsilon}\left\{\left(\sum_{j=j_{0}}^{n-1} \Re\left(\lambda_{j}\right) y^{j}\right) \frac{a}{a^{2}+b^{2}}(y)+\left[\left(-k+\Im\left(\lambda_{0}\right)\right)+\sum_{j=1}^{n-1} \Im\left(\lambda_{j}\right) y^{j}\right] \frac{b}{a^{2}+b^{2}}(y)\right\} \mathrm{d} y
\end{gathered}
$$


$=-\int_{x}^{ \pm \epsilon}\left\{\left(\sum_{j=j_{0}}^{n-1} \Re\left(\lambda_{j}\right) y^{j}\right) \frac{1}{y^{n}} A(y)+\left[\left(-k+\Im\left(\lambda_{0}\right)\right)+\sum_{j=1}^{n-1} \Im\left(\lambda_{j}\right) y^{j}\right] \frac{1}{y^{2 n-m}} B(y)\right\} \mathrm{d} y$,

sendo $A, B \in C^{\infty}\left(A_{\epsilon}\right)$; além disso, $A(0)=1 / a_{0}(0)$ e $B(0)=b_{0}(0) / a_{0}^{2}(0)$. Agora, note que:

- $n-j_{0}>2$. De fato, $m<2 n-1$ implica que $m-n<n-1$; equivalentemente, $m-n+1 \leq n-1$. Logo, $j_{0}+1<n-1$ e, consequentemente, $2<n-j_{0}$.

- $n-j_{0}-(2 n-m)=m-n-j_{0}>0$.

Logo, podemos escrever

$$
\Re\left(-k i C_{0 \pm}(x)+\sum_{j=0}^{n-1} \lambda_{j} C_{j \pm}(x)\right)=-\frac{\Re\left(\lambda_{j_{0}}\right)}{a_{0}(0)} \int_{x}^{ \pm \epsilon} \frac{1}{y^{n-j_{0}}} g_{k}(y) \mathrm{d} y,
$$

sendo

$$
\begin{aligned}
g_{k}(x)=\frac{a_{0}(0)}{\Re\left(\lambda_{j_{0}}\right)}\left\{\left(\sum_{j=j_{0}}^{n-1} \Re\left(\lambda_{j}\right) x^{j-j_{0}}\right)\right. & A(x) \\
& \left.+\left[\left(-k+\Im\left(\lambda_{0}\right)\right)+\sum_{j=1}^{n-1} \Im\left(\lambda_{j}\right) x^{j}\right] x^{m-n-j_{0}} B(x)\right\} .
\end{aligned}
$$

Temos que $g_{k} \in C^{\infty}((-\epsilon, \epsilon))$ e $g_{k}(0)=1$; além disso, podemos encontrar $\epsilon_{k}>0$ tal que

$$
\frac{1}{2} \leq g_{k}(x) \leq \frac{3}{2}, \quad \text { se } \quad x \in\left(-\epsilon_{k}, \epsilon_{k}\right)
$$

Caso (i): Assuma que $\Re\left(\lambda_{j_{0}}\right) a_{0}(0)>0$ e $n-j_{0}$ é ímpar.

Defina

$$
w(x)=\left\{\begin{array}{rrr}
e^{-k i C_{0+}(x)+\sum_{j=0}^{n-1} \lambda_{j} C_{j+}(x)}, & \text { se } & 0<x<\epsilon \\
0, & \text { se } & x=0 \\
e^{-k i C_{0-}(x)+\sum_{j=0}^{n-1} \lambda_{j} C_{j-}(x)}, & \text { se } & -\epsilon<x<0
\end{array}\right.
$$

Mostraremos que $w$ é flat em $x=0$; consequentemente, $w \in C^{\infty}((-\epsilon, \epsilon))$.

Para $0<x<\epsilon_{k}$, temos

$$
\begin{aligned}
|w(x)| & =e^{-\int_{x}^{\epsilon}\left[\sum_{j=j_{0}}^{n-1} \Re\left(\lambda_{j}\right) y^{j} \frac{a}{a^{2}+b^{2}}(y)+\left(-k+\sum_{j=0}^{n-1} \Im\left(\lambda_{j}\right) y^{j}\right) \frac{b}{a^{2}+b^{2}}(y)\right] \mathrm{d} y} \\
& =e^{-\frac{\Re\left(\lambda_{j_{0}}\right)}{a_{0}(0)} \int_{x}^{\epsilon} \frac{1}{y^{n-j_{0}}} g_{k}(y) \mathrm{d} y} e^{-\frac{\Re\left(\lambda_{j_{0}}\right)}{a_{0}(0)} \int_{\epsilon_{k}}^{\epsilon} \frac{1}{y^{n-j_{0}}} g_{k}(y) \mathrm{d} y}
\end{aligned}
$$




$$
\begin{aligned}
& \leq M_{k} e^{-\frac{\Re\left(\lambda_{j_{0}}\right)}{2 a_{0}(0)} \int_{x}^{\epsilon} \frac{1}{\epsilon^{n-j_{0}}} \mathrm{~d} y}=M_{k} e^{\left.\frac{\Re\left(\lambda_{j_{0}}\right)}{2 a_{0}(0)} \frac{1}{\left(n-j_{0}-1\right) y^{n-j_{0}-1}}\right|_{x} ^{\epsilon_{k}}} \\
& =M_{k} e^{\frac{-\Re\left(\lambda_{j_{0}}\right)}{2 a_{0}(0)\left(n-j_{0}-1\right)}\left(\frac{1}{x^{n-j_{0}-1}}-\frac{1}{\epsilon_{k}^{n-j_{0}-1}}\right)}=O\left(|x|^{j}\right), \quad \forall j \in \mathbb{Z}_{+},
\end{aligned}
$$

sendo

$$
M_{k}=e^{-\frac{\Re\left(\lambda_{0}\right)}{a_{0}(0)} \int_{\epsilon_{k}}^{\epsilon} \frac{1}{y^{n-j_{0}}} g_{k}(y) \mathrm{d} y} .
$$

Para $-\epsilon_{k}<x<0$, como $n-j_{0}$ é ímpar, obtemos

$$
\begin{aligned}
& |w(x)|=e^{\int_{-\epsilon_{k}}^{x}\left[\sum_{j=j_{0}}^{n-1} \Re\left(\lambda_{j}\right) y^{j} \frac{a}{a^{2}+b^{2}}(y)+\left(-k+\sum_{j=0}^{n-1} \Im\left(\lambda_{j}\right) y^{j}\right) \frac{b}{a^{2}+b^{2}}(y)\right](y) \mathrm{d} y} \\
& =e^{\frac{(-1)^{n-j_{0}} \Re\left(\lambda_{j_{0}}\right)}{a_{0}(0)} \int_{-\epsilon_{k}}^{x} \frac{1}{(-y)^{n-j_{0}}} g_{k}(y) \mathrm{d} y} e^{\frac{\Re\left(\lambda_{j_{0}}\right)}{a_{0}(0)} \int_{-\epsilon}^{-\epsilon_{k}} \frac{1}{y^{n-j_{0}}} g_{k}(y) \mathrm{d} y} \\
& \leq M_{k}^{\prime} e^{\frac{(-1)^{n-j_{0} \Re\left(\lambda_{j_{0}}\right)}}{2 a_{0}(0)} \int_{-\epsilon_{k}}^{x} \frac{1}{(-y)^{n-j_{0}}} \mathrm{~d} y}=\left.M_{k}^{\prime} e^{\frac{-\Re\left(\lambda_{j_{0}}\right)}{2 a_{0}(0)\left(n-j_{0}-1\right) y^{n-j_{0}-1}}}\right|_{-\epsilon_{k}} ^{x} \\
& =M_{k}^{\prime} e^{\frac{-\Re\left(\lambda_{0}\right)}{2 a_{0}(0)\left(n-j_{0}-1\right)}\left(\frac{1}{x^{n-j_{0}-1}}-\frac{1}{\left(-\epsilon_{k}\right)^{n-j_{0}-1}}\right)}=O\left(|x|^{j}\right), \quad \forall j \in \mathbb{Z}_{+},
\end{aligned}
$$

sendo

$$
M_{k}^{\prime}=e^{\frac{\Re\left(\lambda_{j_{0}}\right)}{a_{0}(0)} \int_{-\epsilon}^{-\epsilon_{k}} \frac{1}{y^{n-j_{0}}} g_{k}(y) \mathrm{d} y} .
$$

Cálculos similares mostram que, para cada $\gamma \in \mathbb{Z}_{+},\left|w^{(\gamma)}(x)\right|=O\left(|x|^{j}\right), \quad \forall j \in \mathbb{Z}_{+}$. Portanto, $w \in C^{\infty}((-\epsilon, \epsilon)), w(x) \neq 0$ se $x \neq 0$, e é flat em $x=0$; consequentemente, $u$ dado por (2.24) tem as propriedades desejadas.

Caso (ii): Assuma que $\Re\left(\lambda_{j_{0}}\right) a_{0}(0)>0$ e $n-j_{0}$ é par.

Defina

$$
w(x)=\left\{\begin{array}{rrr}
e^{-k i C_{0+}(x)+\sum_{j=0}^{n-1} \lambda_{j} C_{j+}(x)}, & \text { se } & 0<x<\epsilon \\
0, & \text { se } & -\epsilon<x \leq 0
\end{array} .\right.
$$

Procedendo como no caso (i) podemos provar que $w \in C^{\infty}((-\epsilon, \epsilon))$; claramente, $w$ é flat em $x=0$ e $w(x) \neq 0$ se $x>0$. Portanto, $u$ dado por (2.24) tem as propriedades desejadas.

Caso (iii): Assuma que $\Re\left(\lambda_{j_{0}}\right) a_{0}(0)<0$ e $n-j_{0}$ é par.

Defina

$$
w(x)=\left\{\begin{array}{rrr}
0, & \text { se } & 0<x<\epsilon \\
e^{-k i C_{0+}(x)+\sum_{j=0}^{n-1} \lambda_{j} C_{j+}(x)}, & \text { se } & -\epsilon<x \leq 0
\end{array} .\right.
$$


Agora, procedendo como no caso (i) podemos provar que, sendo $n-j_{0}$ par, para $-\epsilon_{k}<x<0$ temos

$$
\begin{aligned}
|w(x)| & \leq M_{k}^{\prime} e^{\frac{\Re\left(\lambda_{j_{0}}\right)}{2 a_{0}(0)} \int_{-\epsilon_{k}}^{x} \frac{1}{y^{n-j_{0}}} \mathrm{~d} y}=M_{k}^{\prime} e^{\left.\frac{-\Re\left(\lambda_{j_{0}}\right)}{2 a_{0}(0)\left(n-j_{0}-1\right) y^{n-j_{0}-1}}\right|_{-\epsilon_{k}} ^{x}} \\
& =M_{k}^{\prime} e^{\frac{-\Re\left(\lambda_{0}\right)}{2 a_{0}(0)\left(n-j_{0}-1\right)}\left(\frac{1}{x^{n-j_{0}-1}}-\frac{1}{\left(-\epsilon_{k}\right)^{n-j_{0}-1}}\right)} \\
& =M_{k}^{\prime} e^{\frac{\Re\left(\lambda_{j_{0}}\right)}{2 a_{0}(0)\left(n-j_{0}-1\right)}\left(\frac{1}{(-x)^{n-j_{0}-1}}-\frac{1}{\epsilon_{k}^{n-j_{0}-1}}\right)}=O\left(|x|^{j}\right), \quad \forall j \in \mathbb{Z}_{+},
\end{aligned}
$$

Cálculos similares mostram que, para cada $\gamma \in \mathbb{Z}_{+},\left|w^{(\gamma)}(x)\right|=O\left(|x|^{j}\right), \forall j \in \mathbb{Z}_{+}$. Portanto, $w \in C^{\infty}((-\epsilon, \epsilon))$ é flat em $x=0$ e $w(x) \neq 0$ se $x<0$. Então, $u$ dado por (2.24) tem as propriedades desejadas.

Caso (iv): Assuma que $\Re\left(\lambda_{j_{0}}\right) a_{0}(0)<0$ e $n-j_{0}$ é ímpar.

Seja u uma solução $C^{\infty}$ de (2.15) em uma vizinhança de $\Sigma$. Usando série parcial de Fourier, podemos escrever

$$
u(x, t)=\sum_{k \in \mathbb{Z}} \hat{u}_{k}(x) e^{i k t}
$$

Logo, para cada $k \in \mathbb{Z}$, a equação (2.15) fornece

$$
i k \hat{u}_{k}(x)+(a+i b)(x)\left(\hat{u}_{k}\right)^{\prime}(x)=\sum_{j=0}^{n-1} \lambda_{j} x^{j} \hat{u}_{k}(x) ;
$$

consequentemente, $\hat{u}_{k}$ é dado por

$$
\hat{u}_{k}(x)=\hat{u}_{k}(\epsilon) e^{-k i C_{0+}(x)+\sum_{j=N}^{n-1} \lambda_{j} C_{j+}(x)}, \quad \text { se } \quad 0<x<\epsilon
$$

e

$$
\hat{u}_{k}(x)=\hat{u}_{k}(-\epsilon) e^{-k i C_{0-}(x)+\sum_{j=N}^{n-1} \lambda_{j} C_{j-}(x)}, \quad \text { se } \quad-\epsilon<x<0 .
$$

Agora, fixamos $k \in \mathbb{Z}$.

Para $0<x<\epsilon_{k}$, temos

$$
\begin{aligned}
\left|\hat{u}_{k}(x)\right| & =\left|\hat{u}_{k}(\epsilon)\right| e^{-\int_{x}^{\epsilon}\left[\sum_{j=j_{0}}^{n-1} \Re\left(\lambda_{j}\right) y^{j} \frac{a}{a^{2}+b^{2}}(y)+\left(-k+\sum_{j=0}^{n-1} \Im\left(\lambda_{j}\right) y^{j}\right) \frac{b}{a^{2}+b^{2}}(y)\right] \mathrm{d} y} \\
& =\left|\hat{u}_{k}(\epsilon)\right| e^{-\frac{\Re\left(\lambda_{j_{0}}\right)}{a_{0}(0)} \int_{x}^{\epsilon} \frac{1}{y^{n-j_{0}}} g_{k}(y) \mathrm{d} y} e^{-\frac{\Re\left(\lambda_{0}\right)}{a_{0}(0)} \int_{\epsilon_{k}}^{\epsilon} \frac{1}{y^{n-j_{0}}} g_{k}(y) \mathrm{d} y} \\
& \geq\left|\hat{u}_{k}(\epsilon)\right| M_{k} e^{-\frac{\Re\left(\lambda_{j_{0}}\right)}{2 a_{0}(0)} \int_{x}^{\epsilon} \frac{1}{y^{n-j_{0}}} \mathrm{~d} y}=M_{k} e^{\left.\frac{\Re\left(\lambda_{j_{0}}\right)}{2 a_{0}(0)\left(n-j_{0}-1\right) y^{n-j_{0}-1}}\right|_{x} ^{\epsilon_{k}}}
\end{aligned}
$$




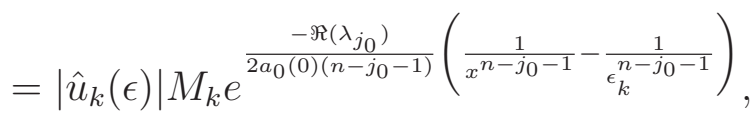

sendo

$$
M_{k}=e^{-\frac{\Re\left(\lambda_{j_{0}}\right)}{a_{0}(0)} \int_{\epsilon_{k}}^{\epsilon} \frac{1}{y^{n-j_{0}}} g_{k}(y) \mathrm{d} y} .
$$

Logo, $\hat{u}_{k}(\epsilon)=0$.

Analogamente, usando que $n-j_{0}$ é ímpar, podemos provar que $\hat{u}_{k}(-\epsilon)=0$.

Portanto, $u \equiv 0$. 


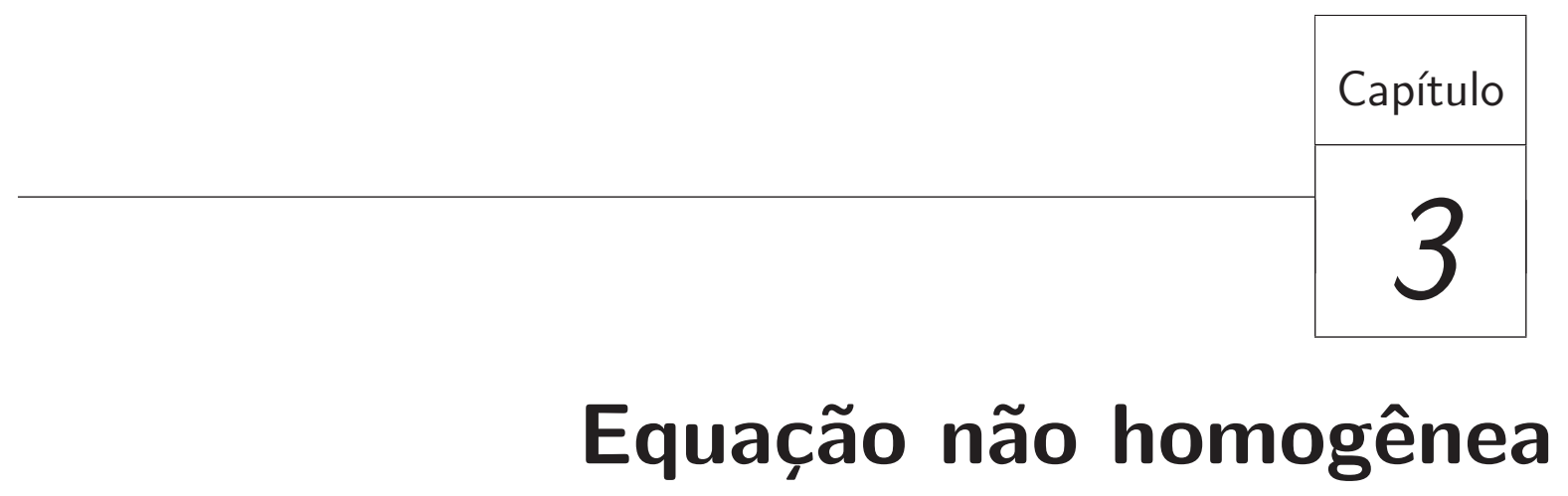

Seja

$$
L=\partial / \partial t+\left(x^{n} a_{0}(x)+i x^{m} b_{0}(x)\right) \partial / \partial x, b_{0} \not \equiv 0,(x, t) \in(-\epsilon, \epsilon) \times S^{1},
$$

um campo vetorial complexo definido em $A_{\epsilon}=(-\epsilon, \epsilon) \times S^{1}$, sendo $a_{0}, b_{0} \in C^{\infty}\left(A_{\epsilon} ; \mathbb{R}\right)$ e $m, n \in \mathbb{Z}_{+}$. Assumimos, como no Capítulo 2, que

- $2 \leq m<2 n-1$

- $b_{0}(0)>0$;

- se a função $a:(-\epsilon, \epsilon) \mapsto \mathbb{R}$, dada por $a(x)=x^{n} a_{0}(x)$, não é flat em $x=0$ então $n$ é a ordem de anulamento de $a$ em $x=0$; se a função $a$ é flat em $x=0$ então $n \geq m$;

- o operador $\mathcal{L}_{p}: C^{\infty}\left(A_{\epsilon}\right) \rightarrow C^{\infty}\left(A_{\epsilon}\right)$ é definido por

$$
\mathcal{L}_{p} u \doteq L u-p u
$$

sendo $p \in C^{\infty}\left(A_{\epsilon}\right)$.

Neste capítulo vamos analisar a resolubilidade de $\mathcal{L}_{p}$ em $\Sigma=\{0\} \times S^{1}$. Veremos que a relação entre as ordens de anulamento das funções $a$ e $b$ e certas médias da função $p$ 
têm influência na existência de solução $u \in C^{\infty}$, em uma vizinhança de $\Sigma=\{0\} \times S^{1}$, da equação

$$
\mathcal{L}_{p} u=f
$$

Tais médias são

$$
\lambda_{j} \doteq \frac{1}{2 \pi} \int_{0}^{2 \pi} p_{j}(t) d t
$$

definidas para cada $j \in \mathbb{Z}_{+}$como no Capítulo 2.

As médias $\lambda_{j}$ também têm influência no número de condições de compatibilidade da equação $\mathcal{L}_{p} u=f$. Para descrever tais condições utilizaremos as funções definidas a seguir.

Para cada $j \in \mathbb{Z}_{+}$, defina

$$
P_{j}(t) \doteq \int_{0}^{t} p_{j}(\sigma) d \sigma
$$

e

$$
Q_{j}(x, t) \doteq \sum_{k=0}^{j} P_{k}(t) x^{k}
$$

\subsection{Existência de solução}

Nesta seção apresentamos condições sobre $p$, sob as quais é possível determinar condições necessárias e suficientes sobre $f$ para que a equação

$$
\mathcal{L}_{p} u=f
$$

tenha solução de classe $C^{\infty}$, em uma vizinhança de $\Sigma$, sendo $\mathcal{L}_{p}$ definido em (3.2) e $p, f \in C^{\infty}\left(A_{\epsilon}\right)$.

Teorema 3.1. Suponha que p satisfaz

$$
\frac{1}{2 \pi i} \int_{0}^{2 \pi} p(0, t) d t \in \mathbb{Z}
$$

$e$

$$
\int_{0}^{2 \pi} \frac{\partial^{j} p}{\partial x^{j}}(0, t) d t=0, j=1, \ldots, r-1
$$

sendo $r \doteq \min \{m, n\}$. Então, dada $f \in C^{\infty}\left(A_{\epsilon}\right)$, a equação (3.3) tem uma solução $u \in C^{\infty}\left(A_{\epsilon}\right)$, em uma vizinhança de $\Sigma$, se, e somente se, $f$ satisfaz

$$
\int_{0}^{2 \pi} \frac{\partial^{j}}{\partial x^{j}}\left(f e^{-Q_{r-1}}\right)(0, t) d t=0, j=0, \ldots, r-1 .
$$


Note que, supondo $p \equiv 0$, as condições (3.1) e (3.8) são satisfeitas. Neste caso o resultado obtido do Teorema 3.1 é precisamente o Teorema 1.6.

Demonstração do Teorema 3.1. Segue de (3.7), (3.8) e do Teorema 1.8, que existe $\varphi \in$ $C^{\infty}\left(A_{\epsilon}\right)$ tal que $L \varphi(x, t)=p(x, t)-p(0, t)$. Note que $\frac{\partial \varphi}{\partial t}(0, t)=0, \forall t \in S^{1}$. Portanto, temos que a função $t \mapsto \varphi(0, t)$ é constante. Então, podemos supor que $\varphi(0, t) \equiv 0$ (substituindo, se necessário, $\varphi(x, t)$ por $\varphi(x, t)-\varphi(0, t))$.

Defina $h: A_{\epsilon} \rightarrow \mathbb{C}$ por $h(x, t) \doteq e^{P_{0}(t)+\varphi(x, t)}$, a qual pertence a $C^{\infty}\left(A_{\epsilon}\right)$. Afirmamos que $u \in C^{\infty}\left(A_{\epsilon}\right)$ é solução da equação $L u=p u+f$ em uma vizinhança de $\Sigma$ se, e somente se, $v=\frac{u}{h}$ é solução de $L v=\frac{f}{h}$ em uma vizinhança de $\Sigma$.

De fato,

$$
\begin{gathered}
L u=p u+f \Longleftrightarrow L(h v)=p h v+f \Longleftrightarrow v L h+h L v=p h v+f \Longleftrightarrow \\
p h v+h L v=p h v+f \Longleftrightarrow h L v=f \Longleftrightarrow \\
L v=\frac{f}{h} .
\end{gathered}
$$

Por outro lado, segue do Teorema 1.8 que a equação $L v=\frac{f}{h}$ tem uma solução $v \in C^{\infty}\left(A_{\epsilon}\right)$, em uma vizinhança de $\Sigma$ se, e somente se, $\frac{f}{h}$ satisfaz

$$
\int_{0}^{2 \pi} \frac{\partial^{j}}{\partial x^{j}}\left(\frac{f}{h}\right)(0, t) d t=0, j=0, \ldots, r-1 .
$$

Afirmamos que, (3.10) é equivalente a (3.9), desde que

$$
\frac{\partial^{j}}{\partial x^{j}}\left(-P_{0}-\varphi+Q_{r-1}\right)(0, t)=0, j=0, \ldots, r-1 .
$$

De fato, de (3.11) temos

$$
\begin{gathered}
\frac{\partial^{j}}{\partial x^{j}}\left[1-e^{-P_{0}-\varphi+Q_{r-1}}\right](0, t)=0, j=0, \ldots, r-1 \Longrightarrow \\
\frac{\partial^{j}}{\partial x^{j}}\left\{f e^{-Q_{r-1}}\left[1-e^{-P_{0}-\varphi+Q_{r-1}}\right]\right\}(0, t)=0, j=0, \ldots, r-1 \Longrightarrow \\
\frac{\partial^{j}}{\partial x^{j}}\left(f e^{-Q_{r-1}}-f e^{-P_{0}-\varphi}\right)(0, t)=0, j=0, \ldots, r-1 \Longrightarrow \\
\frac{\partial^{j}}{\partial x^{j}}\left(f e^{-Q_{r-1}}-\frac{f}{h}\right)(0, t)=0, j=0, \ldots, r-1 \Longrightarrow \\
\int_{0}^{2 \pi} \frac{\partial^{j}}{\partial x^{j}}\left(f e^{-Q_{r-1}}\right)(0, t) d t=\int_{0}^{2 \pi} \frac{\partial^{j}}{\partial x^{j}}\left(\frac{f}{h}\right)(0, t) d t, j=0, \ldots, r-1 .
\end{gathered}
$$


Portanto, para terminar a prova do teorema, é suficiente mostrar que (3.11) ocorre.

Observe que

$$
\begin{gathered}
\frac{\partial}{\partial t}\left(P_{0}+\varphi-Q_{r-1}\right)=p_{0}+p-p_{0}-(a+i b) \frac{\partial \varphi}{\partial x}-\sum_{j=0}^{r-1} p(t) x^{j} \\
=\sum_{j=0}^{r-1} p(t) x^{j}+O\left(x^{r}\right)-x^{r}\left[x^{n-r} a_{0}(x)+i x^{m-r} b_{0}(x)\right] \frac{\partial \varphi}{\partial x}-\sum_{j=0}^{r-1} p(t) x^{j}=O\left(x^{r}\right) .
\end{gathered}
$$

Portanto, integrando ambos os lados em relação a $t$, obtemos

$$
P_{0}(t)+\varphi(x, t)-Q_{r-1}(x, t)-P_{0}(t)-\varphi(0, t)+Q_{r-1}(0, t)=O\left(x^{r}\right)
$$

Como $Q_{r-1}(0, t)=P_{0}(t)$ e $\varphi(0, t)=0$ temos

$$
P_{0}(t)+\varphi(x, t)-Q_{r-1}(x, t)=O\left(x^{r}\right)
$$

consequentemente (3.11) é válido.

De agora em diante, assumiremos que (3.7) ou (3.8) falha. Além disso, fixamos as notações

$$
\alpha_{0} \doteq\left[\left(x^{n-r} a_{0}(x)+i x^{m-r} b_{0}(x)\right]_{x=0},\right.
$$

e

$$
r \doteq \min \{m, n\}
$$

Lema 3.2. Existem únicas constantes $B_{\ell}, \ell=r, \ldots, \ell_{0}+r-2$, tais que

$$
\begin{aligned}
& \int_{0}^{2 \pi} \frac{\partial^{\ell_{0}+r-1}}{\partial x^{\ell_{0}+r-1}}\left[\left(j-\ell_{0}\right) x^{j-1}\left(x^{n} a_{0}+i x^{m} b_{0}\right)\right](0, t) d t \\
& =\sum_{\ell=r+j-1}^{\ell_{0}+r-2} B_{\ell} \int_{0}^{2 \pi} \frac{\partial^{\ell}}{\partial x^{\ell}}\left[\left(j-\ell_{0}\right) x^{j-1}\left(x^{n} a_{0}+i x^{m} b_{0}\right)\right](0, t) d t, \quad j=1, \ldots, \ell_{0}-1 .
\end{aligned}
$$

Demonstração. A matriz associada ao sistema de $\ell_{0}-1$ equações dadas por (3.12) é uma matriz triangular superior cujos elementos da diagonal principal são da forma $2 \pi(j+r-1) !\left(j-\ell_{0}\right) \alpha_{0}, j=1, \ldots, \ell_{0}-1$. Portanto tal sistema tem solução única.

Nosso próximo objetivo será estudar a resolubilidade da equação (3.3) no seguinte sentido. 
Dado $f \in C^{\infty}\left(A_{\epsilon}\right)$, dizemos que a equação (3.3) é resolúvel módulo funções flat se existe $u \in C^{\infty}\left(A_{\epsilon}\right)$ tal que

$$
\mathcal{L}_{p} u-f \text { é flat em } \Sigma \text {. }
$$

Quando isto acontecer escreveremos

$$
\mathcal{L}_{p} u \simeq f
$$

A próxima proposição é uma extensão da Proposição 5.1 em [4] para nosso operador $\mathcal{L}_{p}$

Proposição 3.3. Assuma que (3.7) ou (3.8) falha. Seja $\mathcal{L}_{p}$ dado por (3.2). Com a notação anterior temos:

(1) Se $\lambda_{0} \notin i \mathbb{Z}$, então (3.3) é resolúvel módulo funções flat para cada $f \in C^{\infty}\left(A_{\epsilon}\right)$.

(2) Suponha que $\lambda_{0} \in i \mathbb{Z}$. Seja $N$ o menor inteiro com $1 \leqslant N \leqslant r-1$ tal que $\lambda_{N} \neq 0$. Assuma, além disso, que

$$
1 \leqslant N<r-1
$$

ou então

$$
N=r-1, \quad e \lambda_{r-1}-\ell \alpha_{0} \neq 0, \forall \ell \in \mathbb{Z}_{+} .
$$

Então, (3.3) é resolúvel módulo funções flat se, e somente se, f satisfaz

$$
\int_{0}^{2 \pi} \frac{\partial^{j}}{\partial x^{j}}\left(f e^{-Q_{N-1}}\right)(0, t) d t=0, j=0, \ldots, N-1
$$

(3) Assuma que $\lambda_{0} \in i \mathbb{Z}$. Seja $N$ o menor inteiro com $1 \leqslant N \leqslant r-1$ tal que $\lambda_{N} \neq 0$. Suponha, além disso, que

$$
N=r-1, \quad \text { e } \lambda_{r-1}-\ell_{0} \alpha_{0}=0, \text { para algum } \ell_{0} \in \mathbb{Z}_{+} .
$$

Então, (3.3) é resolúvel módulo funções flat se, e somente se, f satisfaz (3.13) e

$$
\int_{0}^{2 \pi} \frac{\partial^{\ell_{0}+r-1}}{\partial x^{\ell_{0}+r-1}}\left(f e^{-P_{0}-\varphi}\right)(0, t) d t=\sum_{\ell=r}^{\ell_{0}+r-2} B_{\ell} \int_{0}^{2 \pi} \frac{\partial^{\ell}}{\partial x^{\ell}}\left(f e^{-P_{0}-\varphi}\right)(0, t) d t
$$

sendo $B_{\ell}, \ell=r, \ldots, \ell_{0}+r-2$, constantes dadas por (3.12) e $\varphi$ solução da equação

$$
L \varphi=p-p_{0}-\ell_{0} x^{r-1}\left(x^{n-r} a_{0}+i x^{m-r} b_{0}\right)
$$

satisfazendo $\varphi(0, t) \equiv 0 \mathrm{em} S^{1}$. 
Demonstração. Podemos escrever $L$ na forma

$$
L=\frac{\partial}{\partial t}-i x^{r} \beta(x) \frac{\partial}{\partial x}
$$

sendo

$$
\beta(x) \doteq-x^{m-r} b_{0}(x)+i x^{n-r} a_{0}(x) .
$$

Se $m \leqslant n$ então temos $\Re(\beta) \neq 0$ e, procedendo como na prova da Proposição 5.1 de [4], obtemos o resultado desejado. Para $n<m$ temos $\Im(\beta) \neq 0$ e podemos utilizar argumentos similares àqueles da Proposição 5.1 de [4].

Suponha $n<m$. Dividiremos a prova em duas partes.

\section{$1^{a}$ parte:}

Na primeira parte vamos supor $\lambda_{0} \notin i \mathbb{Z}$.

Escreva

$$
f(x, t) \sim \sum_{j \geqslant 0} f_{j}(t) x^{j}
$$

para indicar que $\sum_{j \geqslant 0} f_{j}(t) x^{j}$ é a expansão de Taylor de $f$ com relação a $x$; isto é

$$
f_{j}(t)=\frac{1}{j !} \frac{\partial^{j} f}{\partial x^{j}}(0, t), \quad j=0,1, \ldots
$$

Escrevemos

$$
a_{0}(x) \sim \sum_{j \geqslant 0} a_{0, j} x^{j}, b_{0}(x) \sim \sum_{j \geqslant 0} b_{0, j} x^{j}, p(x, t) \sim \sum_{j \geqslant 0} p_{j}(t) x^{j}, \text { e } u(x, t) \sim \sum_{j \geqslant 0} u_{j}(t) x^{j}
$$

Note que

$$
\begin{aligned}
\left\{x^{n} a_{0}(x)+i x^{m} b_{0}(x)\right\} & \frac{\partial}{\partial x}\left\{\sum_{j \geqslant 0} u_{j}(t) x^{j}\right\} \\
& \sim\left\{x^{n} \sum_{j \geqslant 0} a_{0, j} x^{j}+i x^{m} \sum_{j \geqslant 0} b_{0, j} x^{j}\right\}\left\{\sum_{j \geqslant 1} j u_{j}(t) x^{j-1}\right\} \\
& \sim x^{n} \sum_{j \geqslant 1}\left\{\sum_{\ell=1}^{j} a_{0, j}, \ell \ell u_{\ell}\right\} x^{j-1}+i x^{m} \sum_{j \geqslant 1}\left\{\sum_{\ell=1}^{j} b_{0, j-\ell} \ell u_{\ell}\right\} x^{j-1} \\
& \sim \sum_{j \geqslant 1}\left\{\sum_{\ell=1}^{j} a_{0, j-\ell} \ell u_{\ell}\right\} x^{j+n-1}+\sum_{j \geqslant 1}\left\{\sum_{\ell=1}^{j} i b_{0, j-\ell} \ell u_{\ell}\right\} x^{j+m-1} .
\end{aligned}
$$


Logo,

$$
\begin{aligned}
&\left\{x^{n} a_{0}(x)+i x^{m} b_{0}(x)\right\} \frac{\partial}{\partial x}\left\{\sum_{j \geqslant 0} u_{j}(t) x^{j}\right\} \\
& \sim \sum_{j \geqslant n}\left\{\sum_{\ell=1}^{j-n+1} a_{0, j-n-\ell+1} \ell u_{\ell}\right\} x^{j}+\sum_{j \geqslant m}\left\{\sum_{\ell=1}^{j-m+1} i b_{0, j-m-\ell+1} \ell u_{\ell}\right\} x^{j} .
\end{aligned}
$$

Portanto, de (3.16) concluímos que

$$
\mathcal{L}_{p} u \simeq f
$$

é equivalente a

$$
\begin{aligned}
\sum_{j \geqslant 0} u_{j}^{\prime} x^{j}+\sum_{j \geqslant n}\left\{\sum_{\ell=1}^{j-n+1} a_{0, j-n-\ell+1} \ell u_{\ell}\right\} x^{j}+\sum_{j \geqslant m}\left\{\sum_{\ell=1}^{j-m+1} i b_{0, j-m-\ell+1} \ell u_{\ell}\right\} x^{j} \\
\sim \sum_{j \geqslant 0}\left(\sum_{\ell=0}^{j} p_{\ell} u_{j-\ell}\right) x^{j}+\sum_{j \geqslant 0} f_{j} x^{j} .
\end{aligned}
$$

Assim, obtemos

$$
\begin{gathered}
u_{j}^{\prime}=\sum_{\ell=0}^{j} p_{\ell} u_{j-\ell}+f_{j}, j=0, \ldots, n-1, \\
u_{j}^{\prime}=\sum_{\ell=0}^{j} p_{\ell} u_{j-\ell}+f_{j}-\sum_{\ell=1}^{j-n+1} a_{j-n-\ell+1} \ell u_{\ell}, \quad n \leqslant j<m .
\end{gathered}
$$

e

$$
\begin{aligned}
u_{j}^{\prime}=\sum_{\ell=0}^{j} p_{\ell} u_{j-\ell}+f_{j}- & \sum_{\ell=j-m+2}^{j-n+1} a_{j-n-\ell+1} \ell u_{\ell}(t) \\
& -\sum_{\ell=1}^{j-m+1}\left(a_{j-n-\ell+1}+i b_{j-m-\ell+1}\right) \ell u_{\ell}, j \geqslant m .
\end{aligned}
$$

Vamos determinar $u_{j}$ usando o princípio de indução.

Para $j=0$, de (3.18), obtemos $u_{0}^{\prime}=p_{0} u_{0}+f_{0}$. Então, $\left(u_{0}(t) e^{-P_{0}(t)}\right)^{\prime}=f_{0}(t) e^{-P_{0}(t)}$, e, portanto, $u_{0}(t)=e^{P_{0}(t)} \int_{0}^{t} f_{0}(s) e^{P_{0}(s)} \mathrm{d} s+C_{0} e^{P_{0}(t)}$, para algum $C_{0} \in \mathbb{C}$. Como $C_{0}=u_{0}(0)$ e $u_{0}$ é $2 \pi$-periódica, temos $C_{0}=u_{0}(2 \pi)$ e, assim, $C_{0}$ é determinada de maneira única 
por

$$
C_{0}=\frac{1}{e^{1-P_{0}(2 \pi)}} \int_{0}^{2 \pi} f_{0}(s) e^{P_{0}(2 \pi)-P_{0}(s)} \mathrm{d} s .
$$

De fato, como $\lambda_{0} \notin i \mathbb{Z}$, temos que $1-e^{P_{0}(2 \pi)} \neq 0$; daí,

$$
\begin{gathered}
u_{0}(0)=u_{0}(2 \pi) \Longleftrightarrow C_{0}=e^{P_{0}(2 \pi)} \int_{0}^{2 \pi} f_{0}(s) e^{P_{0}(s)} \mathrm{d} s+C_{0} e^{P_{0}(2 \pi)} \\
\Longleftrightarrow C_{0}=\frac{e^{P_{0}(2 \pi)}}{1-e^{P_{0}(2 \pi)}} \int_{0}^{2 \pi} f_{0}(s) e^{-P_{0}(s)} \mathrm{d} s
\end{gathered}
$$

Portanto, assumindo $\lambda_{0} \notin i \mathbb{Z}$, a equação $u_{0}^{\prime}=p_{0}+f_{0}$ tem uma única solução periódica. Agora, para cada $j \geqslant 1$, assuma que $u_{0}, \ldots, u_{j-1}$ foram determinados de maneira única. Então, podemos definir as funções em $C^{\infty}\left(S^{1}\right)$ dadas por:

$$
\begin{gathered}
\psi_{j} \doteq \sum_{\ell=1}^{j} p_{\ell} u_{j-\ell}+f_{j}, \quad j=0, \ldots, n-1 ; \\
\psi_{j} \doteq \sum_{\ell=1}^{j} p_{\ell} u_{j-\ell}-\sum_{\ell=1}^{j-n+1} a_{0, j-n-\ell+1} \ell u_{\ell}+f_{j}, \quad n \leqslant j<m ; \\
\psi_{j} \doteq \sum_{\ell=1}^{j} p_{\ell} u_{j-\ell}-\sum_{\ell=j-m+2}^{j-n+1} a_{0, j-n-\ell+1} \ell u_{\ell}-\sum_{\ell=1}^{j-m+1}\left(a_{0, j-n-\ell+1}+i b_{0, j-m-\ell+1}\right) \ell u_{\ell}+f_{j}, \quad j \geqslant m .
\end{gathered}
$$

Assim, por (3.18), (3.19) e (3.20), $u_{j}$ deve satisfazer

$$
u_{j}^{\prime}=p_{0} u_{j}+\psi_{j}
$$

Como $\lambda_{0} \notin i \mathbb{Z}$, a equação (3.21) possui única solução $u_{j} \in C^{\infty}\left(S^{1}\right)$ dada por

$$
u_{j}(t)=\int_{0}^{t} \psi_{j}(s) e^{P_{0}(t)-P_{0}(s)} d s+C_{j} e^{P_{0}(t)}
$$

sendo

$$
C_{j}=\frac{1}{1-e^{P_{0}(2 \pi)}} \int_{0}^{2 \pi} \psi_{j}(s) e^{P_{0}(2 \pi)-P_{0}(s)} d s
$$

Finalmente, pelo Teorema de Borel (veja, por exemplo, página 16 de [8]), existe uma função $u \in C^{\infty}\left(A_{\epsilon}\right)$ tal que $\mathcal{L}_{p} u \simeq f$. Portanto, a prova está completa quando $\lambda_{0} \notin i \mathbb{Z}$.

$2^{\underline{a}}$ parte: Suponha que $\lambda_{0} \in i \mathbb{Z}$. A demonstração será feita em quatro etapas.

$1^{a}$ etapa (condições necessárias): 
Vamos provar a necessidade de (3.13) sendo $N$ descrito como no caso (2) ou como no caso (3).

Defina

$$
w(x, t)=u(x, t) e^{-Q_{N-1}(x, t)} .
$$

Note que $e^{P_{0}}$ é $2 \pi$-periódica já que $\lambda_{0} \in i \mathbb{Z}$, e que $P_{1} \ldots, P_{N-1}$ são $2 \pi$-periódicas já que $\lambda_{j} \neq 0$ para $j=1, \ldots, N-1$.

Temos $\mathcal{L}_{p} u \simeq f$ equivalente a

$$
L w+w L\left(Q_{N-1}\right) \simeq p w+f e^{-Q_{N-1}}
$$

consequentemente $\mathcal{L}_{p} u \simeq f$ é equivalente a

$\frac{\partial w}{\partial t}+\left(\sum_{k=0}^{N-1} p_{k}(t) x^{k}\right) w+\left\{x^{n} a_{0}(x)+i x^{m} b_{0}(x)\right\}\left(\frac{\partial w}{\partial x}+\left(\sum_{\nu=1}^{N-1} \nu P_{\nu}(t) x^{\nu-1}\right) w\right) \simeq p w+f e^{-Q_{N-1}}$

a qual podemos escrever como

$$
L w \simeq \widehat{p} w+g,
$$

sendo

$$
g=f e^{-Q_{N-1}},
$$

e

$$
\widehat{p}(x, t)=p(x, t)-\sum_{k=0}^{N-1} p_{k}(t) x^{k}-\left\{x^{n} a_{0}(x)+i x^{m} b_{0}(x)\right\} \sum_{\nu=1}^{N-1} \nu P_{\nu}(t) x^{\nu-1}
$$

Note que

$$
\widehat{p}(x, t) \sim \sum_{k \geqslant N} p_{k}(t) x^{k}-\left\{x^{n} a_{0}(x)+i x^{m} b_{0}(x)\right\} \sum_{\nu=1}^{N-1} \nu P_{\nu}(t) x^{\nu-1} .
$$

Portanto, $w$ satisfaz (3.22) se, e somente se,

$$
\begin{aligned}
& \sum_{j \geqslant 0} w_{j}^{\prime} x^{j}+\sum_{j \geqslant n}\left\{\sum_{\ell=1}^{j-n+1} a_{0, j-n-\ell+1} \ell w_{\ell}\right\} x^{j}+\sum_{j \geqslant m}\left\{\sum_{\ell=1}^{j-m+1} i b_{0, j-m-\ell+1} \ell w_{\ell}\right\} x^{j} \\
\sim & \sum_{j \geqslant N}\left\{\sum_{\ell=N}^{j} p_{\ell} w_{j-\ell}\right\} x^{j}-\left\{\left(x^{n} \sum_{j \geqslant 0} a_{0, j} x^{j}+i x^{m} \sum_{j \geqslant 0} b_{0, j} x^{j}\right) \sum_{\nu=1}^{N-1} \nu P_{\nu} x^{\nu-1}\right\} \sum_{j \geqslant 0} w_{j} x^{j}+\sum_{j \geqslant 0} g_{j} x^{j} .
\end{aligned}
$$


Agora, usando por convenção $a_{0, j}=0=b_{0, j}$ quando $j<0$, temos

$$
\begin{gathered}
\left\{x^{n} \sum_{j \geqslant 0} a_{0, j} x^{j}+i x^{m} \sum_{j \geqslant 0} b_{0, j} x^{j}\right\}\left\{\sum_{\nu=0}^{N-2}(\nu+1) P_{\nu+1} x^{\nu}\right\} \sum_{j \geqslant 0} w_{j} x^{j} \\
\sim\left\{x^{n} \sum_{j \geqslant 0} \sum_{\nu=0}^{N-2}(\nu+1) P_{\nu+1} a_{0, j-\nu} x^{j}+i x^{m} \sum_{j \geqslant 0} \sum_{\nu=0}^{N-2}(\nu+1) P_{\nu+1} b_{0, j-\nu} x^{j}\right\} \sum_{j \geqslant 0} w_{j} x^{j} \\
\sim x^{n} \sum_{j \geqslant 0}\left\{\sum_{\ell=0}^{j} \sum_{\nu=0}^{N-2}(\nu+1) P_{\nu+1} a_{0, \ell-\nu} w_{j-\ell}\right\} x^{j}+i x^{m} \sum_{j \geqslant 0}\left\{\sum_{\ell=0}^{j} \sum_{\nu=0}^{N-2}(\nu+1) P_{\nu+1} b_{0, \ell-\nu} w_{j-\ell}\right\} x^{j} \\
\sim \sum_{j \geqslant 0}\left\{\sum_{\ell=0}^{j} \sum_{\nu=0}^{N-2}(\nu+1) P_{\nu+1} a_{0, \ell-\nu} w_{j-\ell}\right\} x^{j+n}+\sum_{j \geqslant 0}\left\{\sum_{\ell=0}^{j} \sum_{\nu=0}^{N-2}(\nu+1) P_{\nu+1} i b_{0, \ell-\nu} w_{j-\ell}\right\} x^{j+m} \\
\sim \sum_{j \geqslant n}\left\{\sum_{\ell=0}^{j-n} \sum_{\nu=0}^{N-2}(\nu+1) P_{\nu+1} a_{0, \ell-\nu} w_{j-n-\ell}\right\} x^{j}+\sum_{j \geqslant m}\left\{\sum_{\ell=0}^{j-m} \sum_{\nu=0}^{N-2}(\nu+1) P_{\nu+1} i b_{0, \ell-\nu} w_{j-m-\ell}\right\} x^{j} ;
\end{gathered}
$$

logo, (3.22) é equivalente a

$$
\begin{aligned}
& \sum_{j \geqslant 0} w_{j}^{\prime} x^{j}+\sum_{j \geqslant n}\left\{\sum_{\ell=1}^{j-n+1} a_{0, j-n-\ell+1} \ell w_{\ell}\right\} x^{j}+\sum_{j \geqslant m}\left\{\sum_{\ell=1}^{j-m+1} i b_{0, j-m-\ell+1} \ell w_{\ell}\right\} x^{j} \\
& \sim \sum_{j \geqslant N}\left\{\sum_{\ell=N}^{j} p_{\ell} w_{j-\ell}\right\} x^{j}-\sum_{j \geqslant n}\left\{\sum_{\ell=0}^{j-n} \sum_{\nu=0}^{N-2}(\nu+1) P_{\nu+1} a_{0, \ell-\nu} w_{j-n-\ell}\right\} x^{j} \\
&-\sum_{j \geqslant m}\left\{\sum_{\ell=0}^{j-m} \sum_{\nu=0}^{N-2}(\nu+1) P_{\nu+1} i b_{0, \ell-\nu} w_{j-m-\ell}\right\} x^{j}+\sum_{j \geqslant 0} g_{j} x^{j} .
\end{aligned}
$$

Concluímos, assim, que $w$ satisfaz (3.22) se, e somente se,

$$
\begin{aligned}
& w_{j}^{\prime}+\sum_{\ell=1}^{j-m+1}\left(a_{0, j-n-\ell+1}+i b_{0, j-m-\ell+1}\right) \ell w_{\ell}+\sum_{\ell=j-m+2}^{j-n+1} a_{0, j-n-\ell+1} \ell w_{\ell} \\
= & \sum_{\ell=N}^{j} p_{\ell} w_{j-\ell}-\sum_{k=0}^{j-n} \sum_{\nu=0}^{N-2}(\nu+1) P_{\nu+1} a_{0, k-\nu} w_{j-n-k}-i \sum_{k=0}^{j-m} \sum_{\nu=0}^{N-2}(\nu+1) P_{\nu+1} b_{0, k-\nu} w_{j-m-k}+g_{j},
\end{aligned}
$$

para $j=0,1, \ldots$ 
Note que alguns somatórios na última fórmula podem não existir. No caso, se $n \leqslant$ $j<m$ temos (3.23) equivalente a

$$
w_{j}^{\prime}+\sum_{\ell=1}^{j-n+1} a_{0, j-n-\ell+1} \ell w_{\ell}=\sum_{\ell=N}^{j} p_{\ell} w_{j-\ell}-\sum_{k=0}^{j-n} \sum_{\nu=0}^{N-2}(\nu+1) P_{\nu+1} a_{0, k-\nu} w_{j-n-k}+g_{j} .
$$

Quando $N \leqslant j \leqslant n-1$, temos (3.23) equivalente a

$$
w_{j}^{\prime}=\sum_{\ell=N}^{j} p_{\ell} w_{j-\ell}+g_{j}
$$

As $N$ primeiras equações fornecidas por (3.23) são

$$
w_{j}^{\prime}=g_{j}, j=0, \ldots, N-1
$$

as quais possuem soluções periódicas se, e somente se,

$$
\int_{0}^{2 \pi} g_{j}(t) d t=0, j=0, \ldots, N-1
$$

Isso prova a necessidade de (3.13), sendo $N$ descrito como no caso (2) ou como no caso (3).

\section{$2^{a}$ etapa (condições suficientes para o caso (2)):}

Agora, vamos nos concentrar no caso (2) e provar a suficiência de (3.13). Seja $N$ descrito como no caso (2) Assuma que (3.13) seja satisfeita. Então, para $j=0, \ldots, N-$ 1 , a solução geral de (3.26) é

$$
w_{j}(t)=\int_{0}^{t} g_{j}(\sigma) d \sigma+C_{j}
$$

sendo $C_{j}$ uma constante.

A próxima equação, obtida de (3.22), será

$$
w_{N}^{\prime}=p_{N} w_{0}+g_{N}
$$

ou, de forma equivalente

$$
w_{N}^{\prime}=p_{N} C_{0}+p_{N} I_{0}+g_{N},
$$

sendo $I_{0}=\int_{0}^{t} g_{0}(\sigma) d \sigma$. 
Note que (3.27) tem uma solução $w_{N} \in C^{\infty}\left(S^{1}\right)$ se, e somente se,

$$
\int_{0}^{2 \pi}\left\{p_{N} C_{0}+p_{N} I_{0}+g_{N}\right\}(t) d t=0 .
$$

Existe um único valor de $C_{0}$, dado por

$$
C_{0}=\frac{-1}{2 \pi \lambda_{N}}\left(\int_{0}^{2 \pi} p_{N} I_{0} d t+\int_{0}^{2 \pi} g_{N} d t\right),
$$

para o qual (3.28) é satisfeita. Obtemos, assim, $w_{N}(t)=I_{N}(t)+C_{N}$, sendo $C_{N}$ uma constante e $I_{N}(t)=\int_{0}^{t}\left\{p_{N} C_{0}+p_{N} I_{0}+g_{N}\right\}(\sigma) d \sigma$.

Logo, $w_{0}(t)=\int_{0}^{t} g_{0}(\sigma) d \sigma+\frac{-1}{2 \pi \lambda_{N}}\left(\int_{0}^{2 \pi}\left[p_{N} \int_{0}^{t} g_{0}(\sigma) d \sigma+g_{N}\right] d t\right)$. Concluímos assim que $w_{0}$ é unicamente determinada por (3.22).

De forma mais geral, para os índices $j$ tais que $N \leqslant j \leqslant n-1$, provamos por indução em $j$ que $w_{j-N}$ é unicamente determinada por (3.22).

De fato, para $N<j \leqslant n-1$, assuma que $w_{0}, \ldots, w_{j-1-N}$ são unicamente determinadas por (3.22). De (3.25) obtemos a seguinte equação

$$
w_{j}^{\prime}=p_{N} w_{j-N}+\chi_{j}
$$

sendo $\chi_{j} \in C^{\infty}\left(S^{1}\right)$ uma função que depende apenas de $g_{j}$, e das funções $w_{0}, \ldots, w_{j-N-1}$ unicamente determinadas pela hipótese de indução. De forma mais explícita, temos

$$
\chi_{j} \doteq \sum_{\ell=N+1}^{j} p_{\ell} w_{j-\ell}+g_{j}
$$

se $N<j \leqslant n-1$.

A solução geral de (3.29) é $w_{j}(t)=\int_{0}^{t}\left\{p_{N}(\sigma) w_{j-N}(\sigma)+\chi_{j}(\sigma)\right\} d \sigma+C_{j}$. Note que $w_{j}$ está em $C^{\infty}\left(S^{1}\right)$ se, e somente se, $I_{j}(2 \pi)=0$, sendo

$$
I_{j}(t) \doteq \int_{0}^{t}\left\{p_{N}(\sigma) w_{j-N}(\sigma)+\chi_{j}(\sigma)\right\} d \sigma ;
$$

ou, de maneira equivalente se, e somente se,

$$
C_{j-N}=\frac{-1}{2 \pi \lambda_{N}}\left\{\int_{0}^{2 \pi} p_{N} I_{j-N} d t+\int_{0}^{2 \pi} \chi_{j} d t\right\} .
$$


Logo, $w_{j-N}$ é unicamente determinada por (3.22). De fato, temos

$$
w_{j-N}(t)=\int_{0}^{t}\left\{p_{N}(\sigma) w_{j-2 N}(\sigma)+\chi_{j-N}(\sigma)\right\} d \sigma+\frac{-1}{2 \pi \lambda_{N}}\left\{\int_{0}^{2 \pi} p_{N} I_{j-N} d t+\int_{0}^{2 \pi} \chi_{j} d t\right\}
$$

além disso, de $-N \leqslant-1$ obtemos $j-2 N \leqslant j-1-N$. Portanto, assumindo $w_{j-2 N} \equiv 0$ e $\chi_{j-N} \equiv g_{j-N}$ se $j<2 N, w_{j-N}(t)$ é unicamente determinada pela hipótese de indução.

Concluímos assim que (3.22) fornece $w_{j-N}$ de maneira única, quando $N \leqslant j \leqslant n-1$.

Agora, vamos considerar os índices $j$ tais que $j \geqslant n$. Neste caso existem duas situações a serem analisadas:

- $N<n-1$;

- $N=n-1$.

Vamos considerar primeiro o caso em que $N<n-1<j$. Assuma que $w_{0}, \ldots, w_{j-1-N}$ são unicamente determinadas por (3.22). De (3.23), podemos escrever a equação para $w_{j}^{\prime}$ como em (3.29), sendo $\chi_{j}$ unicamente determinada pela igualdade

$$
\begin{aligned}
\chi_{j}=- & \sum_{\ell=1}^{j-m+1}\left(a_{0, j-n-\ell+1}+i b_{0, j-m-\ell+1}\right) \ell w_{\ell}-\sum_{\ell=j-m+2}^{j-n+1} a_{0, j-n-\ell+1} \ell w_{\ell}+\sum_{\ell=N+1}^{j} p_{\ell} w_{j-\ell} \\
& -\sum_{k=0}^{j-n} \sum_{\nu=0}^{N-2}(\nu+1) P_{\nu+1} a_{0, k-\nu} w_{j-n-k}-i \sum_{k=0}^{j-m} \sum_{\nu=0}^{N-2}(\nu+1) P_{\nu+1} b_{0, k-\nu} w_{j-m-k}+g_{j} .
\end{aligned}
$$

Portanto, usando indução em $j$ e procedendo como anteriormente, provamos que $w_{j-N}$ é unicamente determinado por (3.22)

Resta o caso em que $N=n-1<j$. Observe que, da desigualdade $n<m$ temos $\alpha_{0}=a_{0,0}$, e que na situação (2) desta proposição temos $\lambda_{n-1}+\ell a_{0}=\lambda_{r-1}+\ell \alpha_{0} \neq$ $0, \forall \ell \in\{1,2, \ldots\}$.

Assuma que $w_{0}, \ldots, w_{j-1-N}$ são unicamente determinadas por (3.22). De (3.23), podemos escrever a equação para $w_{j}^{\prime}$ na forma

$$
w_{j}^{\prime}=\left\{p_{n-1}-(j-n+1) a_{0,0}\right\} w_{j-n+1}+\chi_{j},
$$

sendo $\chi_{j}$ unicamente determinada (usando a hipótese de indução) pela igualdade

$$
\chi_{j}=-\sum_{\ell=1}^{j-m+1}\left(a_{0, j-n-\ell+1}+i b_{0, j-m-\ell+1}\right) \ell w_{\ell}-\sum_{\ell=j-m+2}^{j-n} a_{0, j-n-\ell+1} \ell w_{\ell}+\sum_{\ell=N+1}^{j} p_{\ell} w_{j-\ell}
$$




$$
-\sum_{k=0}^{j-n} \sum_{\nu=0}^{N-2}(\nu+1) P_{\nu+1} a_{0, k-\nu} w_{j-n-k}-i \sum_{k=0}^{j-m} \sum_{\nu=0}^{N-2}(\nu+1) P_{\nu+1} b_{0, k-\nu} w_{j-m-k}+g_{j} .
$$

A hipótese $\lambda_{n-1}-\ell a_{0,0} \neq 0, \forall \ell \in\{1,2, \ldots\}$ é precisamente o que é necessário para a equação (3.30) ter solução (única) em $C^{\infty}\left(S^{1}\right)$. Mais precisamente, $w_{j-N}$ fica unicamente determinada, usando a hipótese de indução, pela igualdade $w_{j-N}=I_{j-N}+C_{j-N}$, sendo

$$
I_{j-N}=\left\{\begin{array}{l}
\int_{0}^{t} g_{j-n+1}(\sigma) d \sigma, \quad \text { se } j<2 n-2 \\
\int_{0}^{t}\left\{p_{n-1}(\sigma)-(j-2 n+2) a_{0,0}\right\} w_{j-2 n+2}(\sigma)+\chi_{j-n+1}(\sigma) d \sigma, \text { se } 2 n-2 \leqslant j
\end{array}\right.
$$

e

$$
C_{j-N}=\frac{-1}{2 \pi\left\{\lambda_{n-1}-(j-n+1) a_{0,0}\right\}}\left\{\int_{0}^{2 \pi}\left\{p_{n-1}-(j-n+1) a_{0}\right\} I_{j-n+1}+\chi_{j} d t\right\} .
$$

Encerramos assim a prova para o caso (2) desta proposição.

\section{$3^{\underline{a}}$ etapa (condições suficientes para o caso (3));}

Finalmente passaremos para o caso (3) desta proposição. Então $\lambda_{0} \in i \mathbb{Z}, \lambda_{j}=0, j=$ $1, \ldots, n-2$, e existe $\ell_{0} \in \mathbb{Z}_{+}$, tal que $\lambda_{n-1}-\ell_{0} \alpha_{0}=0$.

Defina

$$
q \doteq p-p_{0}-\ell_{0} x^{n-1}\left(a_{0}+i x^{m-n} b_{0}\right)
$$

Sob nossas hipóteses, obtemos

$$
\int_{0}^{2 \pi} \frac{\partial^{j} q}{\partial x^{j}}(0, t) d t=0, j=0, \ldots, n-1 .
$$

Segue de (3.33) e do Teorema 1.8, que existe $\varphi \in C^{\infty}\left(A_{\epsilon}\right)$ tal que $L \varphi(x, t)=q(x, t)$. Note que, $\frac{\partial \varphi}{\partial t}(0, t)=0, \forall t \in S^{1}$. Portanto, temos $t \mapsto \varphi(0, t)$ constante.

Então, podemos supor que $\varphi(0, t) \equiv 0$ (substituindo, se necessário, $\varphi(x, t)$ por $\varphi(x, t)-\varphi(0, t))$.

Defina $h: A_{\epsilon} \rightarrow \mathbb{C}$ por $h(x, t) \doteq e^{P_{0}(t)+\varphi(x, t)}$, a qual pertence $C^{\infty}\left(A_{\epsilon}\right)$. Afirmamos que $u \in C^{\infty}\left(A_{\epsilon}\right)$ satisfaz $\mathcal{L}_{p} u \simeq f$ se, e somente se, $w=\frac{u}{h}$ satisfaz

$$
L w \simeq \ell_{0} x^{n-1}\left(a_{0}+i x^{m-n} b_{0}\right) w+\frac{f}{h} .
$$


De fato,

$$
\begin{gathered}
L u \simeq p u+f \Longleftrightarrow L(h w) \simeq p h w+f \Longleftrightarrow w L h+h L w \simeq p h w+f \Longleftrightarrow \\
w h\left[p-\ell_{0} x^{n-1}\left(a_{0}+i x^{m-n} b_{0}\right)\right]+h L w \simeq p h w+f \Longleftrightarrow \\
-w h \ell_{0} x^{n-1}\left(a_{0}+i x^{m-n} b_{0}\right)+h L w \simeq f \Longleftrightarrow \\
L w \simeq \ell_{0} x^{n-1}\left(a_{0}+i x^{m-n} b_{0}\right) w+\frac{f}{h} .
\end{gathered}
$$

Defina

$$
g=\frac{f}{h} .
$$

Logo, $\mathcal{L}_{p} u \simeq f$ é equivalente a

$$
L w \simeq \ell_{0} x^{n-1}\left(a_{0}+i x^{m-n} b_{0}\right) w+g .
$$

De (3.16) temos

$$
\begin{aligned}
\sum_{j \geqslant 0} w_{j}^{\prime} x^{j}+\sum_{j \geqslant n}\left\{\sum_{\ell=1}^{j-n+1} a_{0, j-n-\ell+1} \ell w_{\ell}\right\} x^{j}+\sum_{j \geqslant m} & \left\{\sum_{\ell=1}^{j-m+1} i b_{0, j-m-\ell+1} \ell w_{\ell}\right\} x^{j} \\
& \sim \ell_{0} x^{n-1}\left(a_{0}+i x^{m-n} b_{0}\right) w+\sum_{j \geqslant 0} g_{j} x^{j}
\end{aligned}
$$

e

$$
\begin{gathered}
\ell_{0} x^{n-1}\left(a_{0}+i x^{m-n} b_{0}\right) w \sim \ell_{0} x^{n-1}\left(a_{0}+i x^{m-n} b_{0}\right) w_{0}+\ell_{0} x^{n}\left(a_{0}+i x^{m-n} b_{0}\right) \sum_{j \geqslant 1} w_{j} x^{j-1} \\
\sim \sum_{j \geqslant n-1} \ell_{0} w_{0} a_{0, j-n+1} x^{j}+\sum_{j \geqslant m-1} \ell_{0} w_{0} i b_{0, j-m+1} x^{j}+\sum_{j \geqslant n}\left\{\sum_{\ell=1}^{j-n+1} a_{0, j-n-\ell+1} \ell_{0} w_{\ell}\right\} x^{j} \\
+\sum_{j \geqslant m}\left\{\sum_{\ell=1}^{j-m+1} i b_{0, j-m-\ell+1} \ell_{0} w_{\ell}\right\} x^{j}
\end{gathered}
$$

concluímos que $w$ satisfaz (3.34) se, e somente se,

$$
w_{j}^{\prime}+\sum_{\ell=1}^{j-m+1}\left(a_{0, j-n-\ell+1}+i b_{0, j-m-\ell+1}\right)\left(\ell-\ell_{0}\right) w_{\ell}+\sum_{\ell=j-m+2}^{j-n+1} a_{0, j-n-\ell+1}\left(\ell-\ell_{0}\right) w_{\ell}=g_{j},
$$

para todo $j \in \mathbb{Z}_{+}$. 
Note que alguns somatórios na última fórmula podem não existir. No caso, se $n \leqslant$ $j<m$ temos (3.35) equivalente a

$$
w_{j}^{\prime}+\sum_{\ell=1}^{j-n+1} a_{0, j-n-\ell+1}\left(\ell-\ell_{0}\right) w_{\ell}=g_{j} .
$$

As $n-2$ primeiras equações, fornecidas por (3.35), são

$$
w_{j}^{\prime}=g_{j}, j=0, \ldots, n-2,
$$

as quais possuem soluções periódicas se, e somente se,

$$
\int_{0}^{2 \pi} \frac{\partial^{j}}{\partial x^{j}}\left(\frac{f}{h}\right)(0, t) d t=0, j=0, \ldots, n-2 .
$$

Portanto, (3.38) é necessária. Afirmamos que (3.38) é equivalente a (3.13). De fato, para mostrar esta equivalência, é suficiente mostrar que

$$
\frac{\partial^{j}}{\partial x^{j}}\left(-P_{0}-\varphi+Q_{n-2}\right)(0, t)=0, j=0, \ldots, n-2
$$

Note que

$$
\begin{aligned}
& \frac{\partial}{\partial t}\left(P_{0}+\varphi-Q_{n-2}\right)=p_{0}+p-p_{0}-\ell_{0} x^{n-1}\left(a_{0}+i x^{m-n} b_{0}\right)-(a+i b) \frac{\partial \varphi}{\partial x}-\sum_{j=0}^{n-2} p_{j}(t) x^{j} \\
& \quad=\sum_{j=0}^{n-2} p_{j}(t) x^{j}+O\left(x^{n-1}\right)-x^{n-1}\left[x a_{0}(x)+i x^{m-n} b_{0}(x)\right] \frac{\partial \varphi}{\partial x}-\sum_{j=0}^{n-2} p_{j}(t) x^{j}=O\left(x^{n-1}\right) .
\end{aligned}
$$

Portanto, integrando com relação a $t$, obtemos

$$
P_{0}(t)+\varphi(x, t)-Q_{n-2}(x, t)-P_{0}(t)-\varphi(0, t)+Q_{n-2}(0, t)=O\left(x^{n-1}\right) .
$$

Como $Q_{n-2}(0, t)=P_{0}(t)$ e $\varphi(0, t)=0$, temos

$$
P_{0}(t)+\varphi(x, t)-Q_{n-2}(x, t)=O\left(x^{n-1}\right)
$$

consequentemente (3.39) é válido. Isso prova que (3.38) é equivalente a (3.13). 
Agora, assuma que (3.13) seja satisfeita. Então, para $j=0, \ldots, n-2$, a solução geral de (3.37) é

$$
w_{j}(t)=\int_{0}^{t} g_{j}(\sigma) d \sigma+C_{j}
$$

sendo $C_{j}$ uma constante.

A próxima equação, obtida de (3.35), será

$$
w_{n-1}^{\prime}=\ell_{0} a_{0,0} w_{0}+g_{n-1}
$$

de forma equivalente,

$$
w_{n-1}^{\prime}=\ell_{0} a_{0,0} C_{0}+\ell_{0} a_{0,0} I_{0}+g_{n-1}
$$

sendo $I_{0}=\int_{0}^{t} g_{0}(\sigma) d \sigma$.

Note que (3.40) tem uma solução $w_{n-1} \in C^{\infty}\left(S^{1}\right)$ se, e somente se,

$$
\int_{0}^{2 \pi}\left\{\ell_{0} a_{0,0} C_{0}+\ell_{0} a_{0,0} I_{0}+g_{n-1}\right\}(t) d t=0
$$

Existe um único valor de $C_{0}$, dado por

$$
C_{0}=-\frac{1}{2 \pi} \int_{0}^{2 \pi} I_{0} d t-\frac{1}{2 \pi \ell_{0} a_{0,0}} \int_{0}^{2 \pi} g_{n-1} d t
$$

para o qual (3.41) é satisfeita. Obtemos, assim, $w_{n-1}(t)=I_{n-1}(t)+C_{n-1}$, sendo $C_{n-1}$ uma constante e $I_{n-1}(t)=\int_{0}^{t}\left\{\ell_{0} a_{0,0} C_{0}+\ell_{0} a_{0,0} I_{0}+g_{n-1}\right\}(\sigma) d \sigma$.

Logo, $w_{0}(t)=\int_{0}^{t} g_{0}(\sigma) d \sigma-\frac{1}{2 \pi} \int_{0}^{2 \pi} I_{0} d t-\frac{1}{2 \pi \ell_{0} a_{0,0}} \int_{0}^{2 \pi} g_{n-1} d t$. Concluímos assim que $w_{0}$ é unicamente determinada por (3.34).

De forma mais geral, para os índices $j \geqslant n-1$, podemos provar por indução em $j$ que $w_{j-n+1}$ é unicamente determinada por (3.34).

De fato, assuma que $w_{0}, \ldots, w_{j-n}$ são unicamente determinadas por (3.34). Por (3.35), podemos escrever a equação para $w_{j}^{\prime}$ na forma

$$
w_{j}^{\prime}=\left[\ell_{0}-(j-n+1)\right] a_{0,0} w_{j-n+1}+\chi_{j},
$$

sendo $\chi_{j} \in C^{\infty}\left(S^{1}\right)$ uma função que depende apenas de $g_{j}$ e das funções $w_{0}, \ldots, w_{j-n}$ (unicamente determinadas pela hipótese de indução). De forma mais explícita, temos

$$
\chi_{j}=-\sum_{\ell=1}^{j-m+1}\left(a_{0, j-n-\ell+1}+i b_{0, j-m-\ell+1}\right)\left(\ell-\ell_{0}\right) w_{\ell}-\sum_{\ell=j-m+2}^{j-n} a_{0, j-n-\ell+1}\left(\ell-\ell_{0}\right) w_{\ell}+g_{j} .
$$


Logo, para $n-1 \leqslant j \leqslant n+\ell_{0}$ é suficiente escolher

$$
C_{j-n+1}=-\frac{1}{2 \pi} \int_{0}^{2 \pi} I_{j-n+1} d t-\frac{1}{2 \pi\left[\ell_{0}-(j-n+1)\right] a_{0,0}} \int_{0}^{2 \pi} \chi_{j} d t .
$$

Assumindo $w_{j-2 n+2} \equiv 0$ e $\chi_{j-n+1} \equiv g_{j-n+1}$ se $n-1<j<2 n-2$, para $n-1 \leqslant j<$ $n+\ell_{0}$ obtemos

$$
\begin{aligned}
w_{j-n+1}(t)=\int_{0}^{t}\left\{\left[\ell_{0}-(j-n\right.\right. & \left.+1)] a_{0,0} w_{j-2 n+2}(\sigma)+\chi_{j-n+1}(\sigma)\right\} d \sigma \\
& -\frac{1}{2 \pi} \int_{0}^{2 \pi} I_{j-n+1} d t-\frac{1}{2 \pi\left[\ell_{0}-(j-n+1)\right] a_{0,0}} \int_{0}^{2 \pi} \chi_{j} d t .
\end{aligned}
$$

Para determinar $w_{\ell_{0}}$ de maneira única, utilizamos a equação que corresponde a $j=$ $n-1+\ell_{0}$, dada por

$$
w_{n-1+\ell_{0}}^{\prime}=\chi_{n-1+\ell_{0}}
$$

a qual tem solução periódica se, e somente se,

$$
\int_{0}^{2 \pi} \chi_{n-1+\ell_{0}} d t=0
$$

sendo que $\chi_{n-1+\ell_{0}}$ já foi unicamente determinada pela hipótese de indução (em outras palavras, todas as constantes $C_{0}, \ldots, C_{\ell_{0}-1}$ já foram unicamente escolhidas).

Afirmamos que (3.44) é uma condição adicional. De fato veremos na $4^{\mathrm{a}}$ etapa da demonstração que (3.44) é uma condição imposta sobre as derivadas de $f$ até ordem $\ell_{0}+n-1$.

Agora, assuma que (3.44) é satisfeita. Então,

$$
w_{n-1+\ell_{0}}=\int_{0}^{t} \chi_{n-1+\ell_{0}}(\sigma) d \sigma+C_{n-1+\ell_{0}} .
$$

Para $j \geqslant n+\ell_{0}$ podemos determinar $w_{j-n+1}$, por indução em $j$, de maneira única pela igualdade $w_{j-n+1}=I_{j-n+1}+C_{j-n+1}$, sendo

$$
I_{j-n+1}=\left\{\begin{array}{l}
\int_{0}^{t} g_{j-n+1}(\sigma) d \sigma, \quad \text { se } j<2 n-2 \\
\int_{0}^{t}\left[\ell_{0}-(j-n+1)\right] a_{0,0} w_{j-2 n+2}(\sigma)+\chi_{j-n+1}(\sigma) d \sigma, \text { se } 2 n-2 \leqslant j
\end{array}\right.
$$


e

$$
C_{j-N}=-\frac{1}{2 \pi} \int_{0}^{2 \pi} I_{j-n+1} d t-\frac{1}{2 \pi\left[\ell_{0}-(j-n+1)\right] a_{0,0}} \int_{0}^{2 \pi} \chi_{j} d t,
$$

já que o coeficiente $\left[\ell_{0}-(j-n+1)\right] a_{0,0}$ é não nulo se $j \geqslant n+\ell_{0}$.

\section{$4^{\underline{a}}$ etapa (interpretação da condição adicional para o caso (3));}

Agora, veremos que a condição adicional (3.44) é equivalente a (3.14). Faremos isto mostrando que, para cada $j=1, \ldots, \ell_{0}-1$ existem constantes $C_{j, \ell}, \ell=n, \ldots, j+n$, de forma que

$$
\int_{0}^{2 \pi} w_{j}(t) d t=\sum_{\ell=n}^{j+n-1} C_{j, \ell} \int_{0}^{2 \pi} g_{\ell}(t) d t
$$

Provaremos (3.47) usando indução em $j$.

De (3.42), obtemos

$$
\int_{0}^{2 \pi} w_{1}(t) d t=\frac{1}{\left(1-\ell_{0}\right) a_{0,0}} \int_{0}^{2 \pi} g_{n}(t) d t
$$

Portanto (3.47) é satisfeita para $j=1$. Seja $k \in \mathbb{Z}_{+}$tal que $2 \leqslant k \leqslant \ell_{0}-1$. Suponha que (3.47) seja satisfeira para $j=1, \ldots, k-1$, mostraremos que (3.47) também é satisfeita para $j=k$.

De (3.35) obtemos

$$
\begin{aligned}
\int_{0}^{2 \pi} w_{k+n-1}^{\prime}(t) d t+a_{0,0}\left(k-\ell_{0}\right) \int_{0}^{2 \pi} w_{k}(t) d t=\int_{0}^{2 \pi} g_{k+n-1}(t) d t \\
-\sum_{\ell=1}^{k+n-m}\left(a_{0, k-\ell}+i b_{0, k+n-m-\ell}\right)\left(\ell-\ell_{0}\right) \int_{0}^{2 \pi} w_{\ell}(t) d t-\sum_{\ell=k+n-m+1}^{k-1} a_{0, k-\ell}\left(\ell-\ell_{0}\right) \int_{0}^{2 \pi} w_{\ell}(t) d t .
\end{aligned}
$$

Portanto, usando a hipótese de indução, temos

$$
\begin{aligned}
& \int_{0}^{2 \pi} w_{k}(t) d t=\frac{1}{a_{0,0}\left(k-\ell_{0}\right)} \int_{0}^{2 \pi} g_{k+n-1}(t) d t \\
& -\frac{1}{a_{0,0}\left(k-\ell_{0}\right)} \sum_{\ell=1}^{k+n-m}\left(a_{0, k-\ell}+i b_{0, k+n-m-\ell}\right)\left(\ell-\ell_{0}\right) \sum_{\nu=n}^{\ell+n-1} C_{\ell, \nu} \int_{0}^{2 \pi} g_{\nu}(t) d t \\
& \quad-\frac{1}{a_{0,0}\left(k-\ell_{0}\right)} \sum_{\ell=k+n-m+1}^{k-1} a_{0, k-\ell}\left(\ell-\ell_{0}\right) \sum_{\nu=n}^{\ell+n-1} C_{\ell, \nu} \int_{0}^{2 \pi} g_{\nu}(t) d t .
\end{aligned}
$$


Agora, reordenando a expressão à direita da equação anterior, encontramos as constantes $C_{k, \ell}, \ell=n, \ldots, k+n-1$, de forma que

$$
\int_{0}^{2 \pi} w_{k}(t) d t=\sum_{\ell=n}^{k+n-1} C_{k, \ell} \int_{0}^{2 \pi} g_{\ell}(t) d t
$$

e encerramos o processo de indução.

De (3.47), podemos reescrever (3.44) na forma

$$
\begin{aligned}
\int_{0}^{2 \pi} g_{\ell_{0}+n-1}(t) d t=\sum_{\ell=1}^{\ell_{0}+n-m}\left(a_{0, \ell_{0}-\ell}\right. & \left.+i b_{0, \ell_{0}+n-m-\ell}\right)\left(\ell-\ell_{0}\right) \sum_{\nu=n}^{\ell+n-1} C_{\ell, \nu} \int_{0}^{2 \pi} g_{\nu}(t) d t \\
& +\sum_{\ell=\ell_{0}+n-m+1}^{\ell_{0}-1} a_{0, \ell_{0}-\ell}\left(\ell-\ell_{0}\right) \sum_{\nu=n}^{\ell+n-1} C_{\ell, \nu} \int_{0}^{2 \pi} g_{\nu}(t) d t .
\end{aligned}
$$

Agora, estamos prontos para descrever a condição adicional na forma desejada; reordenamos a expressão à direita da equação anterior para encontrarmos constantes $B_{\ell}, \ell=n, \ldots, \ell_{0}+n-1$, de forma que (3.44) seja equivalente a

$$
\int_{0}^{2 \pi} \frac{\partial^{\ell_{0}+n-1}}{\partial x^{\ell_{0}+n-1}}\left(f e^{-P_{0}-\varphi}\right)(0, t) d t=\sum_{\ell=n}^{\ell_{0}+n-2} B_{\ell} \int_{0}^{2 \pi} \frac{\partial^{\ell}}{\partial x^{\ell}}\left(f e^{-P_{0}-\varphi}\right)(0, t) d t
$$

Note que as constantes $B_{\ell}, \ell=n, \ldots, \ell_{0}+n-1$ não dependem da função $f$; dependem apenas da função $a+i b$ e são determinadas por (3.12).

De fato, seja $u_{j} \doteq x^{j} e^{\varphi+P_{0}} \in C^{\infty}\left(A_{\epsilon}\right), j=1, \ldots, \ell_{0}-1$, então

$$
\begin{aligned}
L u_{j} & =x^{j} L e^{\varphi+P_{0}}+e^{\varphi+P_{0}} L x^{j} \\
& =u_{j} L\left(\varphi+P_{0}\right)+e^{\varphi+P_{0}} j x^{j}\left(x^{n-1} a_{0}+i x^{m-1} b_{0}\right) \\
& =p u_{j}+e^{\varphi+P_{0}}\left(j-\ell_{0}\right) x^{j}\left(x^{n-1} a_{0}+i x^{m-1} b_{0}\right) ;
\end{aligned}
$$

concluímos que $e^{\varphi+P_{0}}\left(j-\ell_{0}\right) x^{j-1}\left(x^{n} a_{0}+i x^{m} b_{0}\right)$ está na imagem de $\mathcal{L}_{p}$. Logo (3.12) segue de (3.14).

Afirmamos que para tratar os casos nos quais (3.7) ou (3.8) falham, a resolubilidade de (3.3) é reduzida ao caso em que $f$ é flat em $\Sigma$. De fato, sob as hipóteses da Proposição 3.3, existe $v \in C^{\infty}\left(A_{\epsilon}\right)$ tal que $g \doteq \mathcal{L}_{p} v-f$ é flat em $\Sigma$. Logo, se $\mathcal{L}_{p} u=g$ tem solução de classe $C^{\infty}$ então $w \doteq u-v$ é de classe $C^{\infty}$ e satisfaz

$$
\mathcal{L}_{p} w=\mathcal{L}_{p}(u-v) \mathcal{L}_{p} u-\mathcal{L}_{p} v=g-g+f
$$




$$
=f .
$$

O próximo resultado, cuja demonstração é uma adaptação daquela da Proposição 5.2 em [4], permite simplificar o termo de ordem zero do nosso operador.

Proposição 3.4. Seja $\mathcal{L}_{p}$ dado por (3.2) e sejam $\lambda_{1}, \ldots, \lambda_{r-1}$ dados por (3.4). A equação

$$
L v=\left(\sum_{j=0}^{r-1} \lambda_{j} x^{j}\right) v+h, \quad r=\min \{n, m\},
$$

tem uma solução $v \in C^{\infty}\left(A_{\epsilon}\right)$ para cada $h$ flat em $\Sigma$ se, e somente se, (3.3) tem uma solução $u \in C^{\infty}\left(A_{\epsilon}\right)$ para cada f flat em $\Sigma$.

Demonstração. Usando fórmula de Taylor podemos escrever

$$
p(x, t)=\sum_{j=0}^{r-1} p_{j}(t) x^{j}+x^{r} \chi(x, t),
$$

$\operatorname{com} \chi \in C^{\infty}\left(A_{\epsilon}\right)$

Defina

$$
\begin{aligned}
\tilde{p}_{j}(t) & \doteq p_{j}(t)-\lambda_{j}, j=0, \ldots, r-1, \\
\tilde{P}_{j}(t) & \doteq \int_{0}^{t} \tilde{p}_{j}(\sigma) d \sigma, j=0, \ldots, r-1
\end{aligned}
$$

$\mathrm{e}$

$$
\tilde{Q}_{r-1}(x, t) \doteq \sum_{j=0}^{r-1} \tilde{P}_{j}(t) x^{j} .
$$

Também, para cada $f \in C^{\infty}\left(A_{\epsilon}\right)$, flat em $\Sigma$, definamos

$$
g \doteq f e^{-\tilde{Q}_{r-1}}
$$

Note que $g$ é flat e que $\tilde{P}_{0}(t), \ldots, \tilde{P}_{r-1}(t)$ são $2 \pi$-periódicas.

Afirmamos que $u$ é solução de $\mathcal{L}_{p} u=f$ se, e somente se,

$$
w(x, t) \doteq u(x, t) e^{-\tilde{Q}_{r-1}(x, t)},
$$

é solução de

$$
L w=g+\left(p-L\left(\tilde{Q}_{r-1}\right)\right) w .
$$


De fato,

$$
\begin{gathered}
\mathcal{L}_{p} u=f \Longleftrightarrow \\
L u=p u+f \Longleftrightarrow \\
f+p w e^{\tilde{Q}_{r-1}}=L\left(w e^{\tilde{Q}_{r-1}}\right)=L w e^{\tilde{Q}_{r-1}}+L\left(\tilde{Q}_{r-1}\right) w e^{\tilde{Q}_{r-1}} \Longleftrightarrow \\
g+p w=L w+L\left(\tilde{Q}_{r-1}\right) w \Longleftrightarrow \\
L w=g+\left(p-L\left(\tilde{Q}_{r-1}\right)\right) w .
\end{gathered}
$$

Agora,

$$
\begin{gathered}
p-L\left(\tilde{Q}_{r-1}\right)=p(x, t)-\sum_{j=0}^{r-1} \tilde{p}_{j}(t) x^{j}-x^{r}\left\{x^{n-r} a_{0}(x)+i x^{m-r} b_{0}(x)\right\} \sum_{\nu=1}^{r-1} \nu \tilde{P}_{\nu}(t) x^{\nu-1} \\
=\sum_{j=0}^{r-1} \lambda_{j} x^{j}+p^{\#}(x, t)
\end{gathered}
$$

sendo

$$
p^{\#}(x, t)=x^{r}\left\{\chi(x, t)-i\left[x^{n-r} a_{0}(x)+i x^{m-r} b_{0}(x)\right] \sum_{\nu=1}^{r-1} \nu P_{\nu}(t) x^{\nu-1}\right\} .
$$

Note que $p^{\#}(x, t)$ satisfaz

$$
\int_{0}^{2 \pi} \frac{\partial^{j} p^{\#}}{\partial x^{j}}(0, t) d t=0, j=0, \ldots, r-1
$$

Logo pelo Teorema 1.8 podemos encontrar $\varphi \in C^{\infty}\left(A_{\epsilon}\right)$ tal que $L \varphi=p^{\#}$ em uma vizinhança de $\Sigma$. Seja $v=w e^{-\varphi}$ e $h=g e^{-\varphi}$. Então, (3.49) é equivalente a

$$
L v=\left(\sum_{j=0}^{r-1} \lambda_{j} x^{j}\right) v+h .
$$

Note que a função $h=f e^{-\varphi-\tilde{Q}_{r-1}}$ é flat em $\Sigma$ se, e somente se, $f$ é flat em $\Sigma$.

No que segue vamos nos concentrar no estudo da resolubilidade da equação

$$
L u=\left(\sum_{j=0}^{r-1} \lambda_{j} x^{j}\right) u+f
$$

com $f$ flat em $\Sigma$.

Começamos com dois lemas técnicos: 
Lema 3.5. Assuma que (3.7) ou (3.8) falha. Defina o inteiro $j_{0}$ como segue:

(i) se $\Re\left(\lambda_{j}\right)=0$ para cada $j \in\{0, \cdots, r-1\}$, seja $j_{0}=r-1$;

(ii) se $\Re\left(\lambda_{j}\right) \neq 0$ para algum $j \in\{0, \cdots, r-1\}$, seja $j_{0}=\min _{0 \leqslant j \leqslant r-1}\left\{j: \Re\left(\lambda_{j}\right) \neq 0\right\}$.

Suponha que $m-n \leqslant j_{0}$, que $0<x<\epsilon$ e que $0<y<\epsilon$. Dado $k \in \mathbb{Z}$, se $(y-x) D_{k}>0$, então existe $M>0$ e existe $\ell>1$ tais que

$$
\left|\frac{e^{-i k \int_{x}^{\epsilon} \frac{1}{a+i b}(s) \mathrm{d} s+\sum_{j=0}^{r-1} \lambda_{j} \int_{x}^{\epsilon} \frac{s^{j}}{a+i b}(s) \mathrm{d} s}}{e^{-i k \int_{y}^{\epsilon} \frac{1}{a+i b}(s) \mathrm{d} s+\sum_{j=0}^{r-1} \lambda_{j} \int_{y}^{\epsilon} \frac{s^{j}}{a+i b}(s) \mathrm{d} s}}\right| \leqslant e^{\frac{-D_{k} M}{(\ell-1)}\left(\frac{1}{x^{\ell-1}}-\frac{1}{y^{\ell-1}}\right)},
$$

sendo

$$
D_{k}=\Re\left(\lambda_{j_{0}}\right)\left[x^{n-m+j_{0}} a_{0}(x)\right]_{x=0}+\left(\Im\left(\lambda_{0}\right)-k\right) b_{0}(0) .
$$

Demonstração. Defina

$$
\begin{aligned}
g_{k}(x) \doteq \frac{1}{D_{k}}\left[\left(\sum_{j=j_{0}}^{r-1} \Re\left(\lambda_{j}\right) x^{j-m+n}\right)\right. & \frac{a_{0}}{b_{0}}(x) \\
& \left.\quad k+\Im\left(\lambda_{0}\right)+\sum_{j=1}^{r-1} \Im\left(\lambda_{j}\right) x^{j}\right] x^{m+\ell} \frac{b_{0}}{a^{2}+b^{2}}(x) .
\end{aligned}
$$

Então, procedendo como na prova da Proposição 2.3 (veja (2.21)), para $x>0$, obtemos

$$
\left|e^{-i k \int_{x}^{\epsilon} \frac{1}{a+i b}(s) \mathrm{d} s+\sum_{j=0}^{r-1} \lambda_{j} \int_{x}^{\epsilon} \frac{s^{j}}{a+i b}(s) \mathrm{d} s}\right|=e^{-D_{k} \int_{x}^{\epsilon} \frac{1}{s^{\ell}} g_{k}(s) \mathrm{d} s},
$$

sendo

$$
\ell=\left\{\begin{array}{rl}
m, & \text { if } \quad 2 \leq m<n \\
2 n-m, & \text { if } \quad n \leq m<2 n-1
\end{array} .\right.
$$

Como $x^{m+\ell} \frac{b_{0}}{a^{2}+b^{2}} \in C^{\infty}((-\epsilon, \epsilon))$ e $\left[x^{m+\ell} \frac{b_{0}}{a^{2}+b^{2}}(x)\right]_{x=0}>0$, existem $\alpha^{\prime}, \beta^{\prime}>0$ tais que

$$
\alpha^{\prime} \leqslant x^{m+\ell} \frac{b_{0}}{a^{2}+b^{2}}(x) \leqslant \beta^{\prime},
$$

para $\epsilon>0$ suficientemente pequeno.

Defina

$$
g(x) \doteq\left[\left(\sum_{j=j_{0}}^{r-1} \Re\left(\lambda_{j}\right) x^{j-m+n}\right) \frac{a_{0}}{b_{0}}(x)+\sum_{j=1}^{r-1} \Im\left(\lambda_{j}\right) x^{j}\right] x^{m+\ell} \frac{b_{0}}{a^{2}+b^{2}}(x) .
$$


Note que $g \in C^{\infty}((-\epsilon, \epsilon))$ e é limitada. Logo, existe $M^{\prime}>0$ tal que

$$
|g(x)| \leqslant M^{\prime}
$$

Além disso, existe $n_{0} \in \mathbb{N}$ tal que

$$
\left|D_{k}\right| \geqslant \frac{4 b_{0}(0) M^{\prime}}{\alpha^{\prime}}
$$

e

$$
\frac{1}{2 b_{0}(0)} \leqslant \frac{\Im\left(\lambda_{0}\right)-k}{D_{k}} \leqslant \frac{2}{b_{0}(0)}
$$

para $|k|>n_{0}$.

Logo, de (3.52) e (3.53), obtemos

$$
\frac{-\alpha^{\prime}}{4 b_{0}(0)} \leqslant \frac{g(x)}{D_{k}} \leqslant \frac{\alpha^{\prime}}{4 b_{0}(0)}
$$

para $|k|>n_{0}$.

Além disso, de (3.51) e (3.54), obtemos

$$
\frac{\alpha^{\prime}}{2 b_{0}(0)} \leqslant \alpha^{\prime} \frac{\Im\left(\lambda_{0}\right)-k}{D_{k}} \leqslant \frac{\Im\left(\lambda_{0}\right)-k}{D_{k}} x^{m+\ell} \frac{b_{0}}{a^{2}+b^{2}}(x) \leqslant \beta^{\prime} \frac{\Im\left(\lambda_{0}\right)-k}{D_{k}} \leqslant \frac{2 \beta^{\prime}}{b_{0}(0)},
$$

para $|k|>n_{1}$.

Logo, assumindo $\epsilon>0$ suficientemente pequeno, para $\alpha=\frac{\alpha^{\prime}}{4 b_{0}(0)}$ e $\beta=\frac{2 \beta^{\prime}}{b_{0}(0)}+\frac{\alpha^{\prime}}{4 b_{0}(0)}$ temos

$$
\alpha \leqslant g_{k}(x) \leqslant \beta
$$

para cada $k \in \mathbb{Z}$, já que

$$
g_{k}(x)=\frac{g(x)}{D_{k}}+\frac{\Im\left(\lambda_{0}\right)-k}{D_{k}} x^{m+\ell} \frac{b_{0}}{a^{2}+b^{2}}(x) .
$$

Portanto, de $(3.50)$, se $(y-x) D_{k}>0$ então

$$
\begin{aligned}
\mid \frac{e^{-i k \int_{x}^{\epsilon} \frac{1}{a+i b}(s) \mathrm{d} s+\sum_{j=0}^{r-1} \lambda_{j} \int_{x}^{\epsilon} \frac{s^{j}}{a+i b}(s) \mathrm{d} s}}{e^{-i k \int_{y}^{\epsilon} \frac{1}{a+i b}(s) \mathrm{d} s+\sum_{j=0}^{r-1} \lambda_{j} \int_{y}^{\epsilon} \frac{s^{j}}{a+i b}(s) \mathrm{d} s} \mid} & =e^{-D_{k} \int_{x}^{y} \frac{1}{s^{\ell}} g_{k}(s) \mathrm{d} s} \\
& \leqslant e^{\frac{-\left(D_{k}\right) \alpha}{(\ell-1)}\left(\frac{1}{x^{\ell-1}}-\frac{1}{y^{\ell-1}}\right)} .
\end{aligned}
$$


Lema 3.6. Assuma que (3.7) ou (3.8) falha. Defina o inteiro $j_{0}$ como segue:

(i) se $\Re\left(\lambda_{j}\right)=0$ para cada $j \in\{0, \cdots, r-1\}$, seja $j_{0}=r-1$;

(ii) se $\Re\left(\lambda_{j}\right) \neq 0$ para algum $j \in\{0, \cdots, r-1\}$, seja $j_{0}=\min _{0 \leqslant j \leqslant r-1}\left\{j: \Re\left(\lambda_{j}\right) \neq 0\right\}$.

Suponha que $m-n \leqslant j_{0}$ e que existe $k \in \mathbb{Z}$ tal que $k=\Im\left(\lambda_{0}\right)+\Re\left(\lambda_{j_{0}}\right)\left[x^{n-m+j_{0}} \frac{a_{0}(x)}{b_{0}(x)}\right]_{x=0}$. Então, para $h \in C^{\infty}((-\epsilon, \epsilon))$, flat em $x=0$, existe $v \in C^{\infty}((-\epsilon, \epsilon))$ tal que

$$
i k v(x)+(a+i b)(x) v^{\prime}(x)=\left(\sum_{j=0}^{r-1} \lambda_{j} x^{j}\right) v(x)+h(x)
$$

em uma vizinhança de $x=0$.

Demonstração. Primeiro vamos nos concentrar na região $x>0$.

Como já vimos anteriormente,

$$
w(x)=e^{i k C_{0+}(x)-\sum_{j=0}^{r-1} \lambda_{j} C_{j+}(x)},
$$

é uma solução de

$$
(a+i b)(x) w^{\prime}(x)=i k w(x)-\left(\sum_{j=0}^{r-1} \lambda_{j} x^{j}\right) w(x),
$$

em $I_{\epsilon}^{+}=(0, \epsilon)$.

Então, para $x>0$, a equação

$$
i k v(x)+(a+i b)(x) v^{\prime}(x)=\left(\sum_{j=0}^{r-1} \lambda_{j} x^{j}\right) v(x)+h(x)
$$

fornece

$$
[v(x) w(x)]^{\prime}=w(x) \frac{h}{a+i b}(x) .
$$

Logo, para cada $x>0$, se existe $j_{1} \in\{0,1, \ldots\}$ tal que $y^{j_{1}} w(y)$ é limitada em $I_{\epsilon}^{+}$, então determinamos

$$
v(x)=\int_{0}^{x} \frac{w(y)}{w(x)} \frac{h}{a+i b}(y) \mathrm{d} y
$$

se não existe $j_{1} \in\{0,1, \ldots\}$ tal que $y^{j_{1}} w(y)$ é limitada em $I_{\epsilon}^{+}$, então determinamos

$$
v(x)=-\int_{x}^{\epsilon} \frac{w(y)}{w(x)} \frac{h}{a+i b}(y) \mathrm{d} y .
$$


Agora, vamos estudar $|w|$.

Note que

$$
\begin{aligned}
& \Re\left(i k C_{0}(x)-\sum_{j=0}^{r-1} \lambda_{j} C_{j}(x)\right) \\
& \quad=\int_{x}^{\epsilon}\left[\left(\sum_{j=j_{0}}^{r-1} \Re\left(\lambda_{j}\right) y^{j-m+n}\right) \frac{a_{0}}{b_{0}}(y)-k+\Im\left(\lambda_{0}\right)+\sum_{j=1}^{r-1} \Im\left(\lambda_{j}\right) y^{j}\right] \frac{b}{a^{2}+b^{2}}(y) \mathrm{d} y .
\end{aligned}
$$

Como $k=\Im\left(\lambda_{0}\right)+\Re\left(\lambda_{j_{0}}\right)\left[x^{n-m+j_{0}} \frac{a_{0}(x)}{b_{0}(x)}\right]_{x=0}$, existe $\ell_{0} \in \mathbb{Z}_{+}$e existe $\chi \in C^{\infty}((-\epsilon, \epsilon))$ de forma que

$$
\sum_{j=j_{0}}^{r-1} \Re\left(\lambda_{j}\right) y^{j+n-m} \frac{a_{0}}{b_{0}}(y)-k+\sum_{j=0}^{r-1} \Im\left(\lambda_{j}\right) y^{j}=y^{\ell_{0}} \chi(y) .
$$

Se a ordem de anulamento de $\sum_{j=j_{0}}^{r-1} \Re\left(\lambda_{j}\right) y^{j+n-m} \frac{a_{0}}{b_{0}}(y)-k+\sum_{j=0}^{r-1} \Im\left(\lambda_{j}\right) y^{j}$ em $y=0$ é finita, vamos assumir que tal ordem é $\ell_{0}$; se $\sum_{j=j_{0}}^{r-1} \Re\left(\lambda_{j}\right) y^{j+n-m} \frac{a_{0}}{b_{0}}(y)-k+\sum_{j=0}^{r-1} \Im\left(\lambda_{j}\right) y^{j}$ é flat em $y=0$, tomamos $\ell_{0}$ tal que

$$
\ell_{0}+1 \geqslant \ell \doteq\left\{\begin{array}{ll}
m, & \text { se } 2 \leqslant m<n \\
2 n-m, & \text { se } n \leqslant m<2 n-1
\end{array} .\right.
$$

O estudo de $|w|$ será dividido em três casos:

(a1) $\ell-\ell_{0} \leqslant 1$;

(a2) $\ell-\ell_{0}>1$ e $\chi(0)<0$

(a3) $\ell-\ell_{0}>1$ e $\chi(0)>0$.

No que segue, vamos demonstrar que se (a1) ou (a2) ocorrem, então existe $j_{1} \in$ $\{0,1, \ldots\}$, tal que $y^{j_{1}} w(y)$ é limitada em $I_{\epsilon}^{+}$.

Quando (a1) ocorre, para $0<y<\epsilon$, temos

$$
\begin{aligned}
& |w(y)|=e^{\int_{y}^{\epsilon}\left(\sum_{j=j_{0}}^{r-1} \Re\left(\lambda_{j}\right) s^{j+n-m} \frac{a_{0}}{b_{0}}(s)-k+\sum_{j=0}^{r-1} \Im\left(\lambda_{j}\right) s^{j}\right) \frac{b_{0}}{s^{2 n-m} a_{0}^{2}+s^{m} b_{0}^{2}}(s) \mathrm{d} s}
\end{aligned}
$$

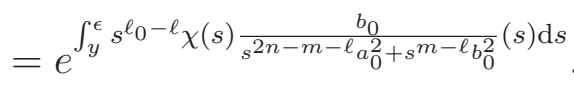

Como $\chi(s) \frac{b_{0}}{s^{2 n-m-\ell} a_{0}^{2}+s^{m-\ell} b_{0}^{2}}(s) \in C^{\infty}((-\epsilon, \epsilon))$, existe $M_{2} \in \mathbb{R}$ tal que

$$
|w(y)| \leqslant e^{M_{2} \int_{y}^{\epsilon} s^{\ell_{0}-\ell} \mathrm{d} s} .
$$


Logo, quando (a1) ocorre, para cada $0<y<\epsilon$ temos

- $|w(y)| \leqslant e^{\frac{M_{2}}{\left(\ell_{0}-\ell+1\right)}\left(\epsilon^{\ell_{0}-\ell+1}-y^{\ell_{0}-\ell+1}\right)} \leqslant e^{\frac{M_{2}}{\ell_{0}-\ell+1} \epsilon^{\ell_{0}-\ell+1}}, \quad$ se $\quad \ell_{0}-\ell>-1$;

- $|w(y)| \leqslant e^{\ln \left(\frac{\epsilon}{y}\right)^{M_{2}}}=\left(\frac{\epsilon}{y}\right)^{M_{2}}, \quad$ se $\quad \ell_{0}-\ell=-1$.

Defina

$$
j_{1} \doteq\left\{\begin{array}{ll}
0, & \text { se } \ell-\ell_{0}<1 \\
M_{2}, & \text { se } \ell-\ell_{0}=1
\end{array} .\right.
$$

Logo, concluímos que $y^{j_{1}} w(y)$ é limitada em $I_{\epsilon}^{+}$.

Agora, suponha que (a2) seja verdadeira. Para $0<y<\epsilon$, temos

$$
\begin{aligned}
& |w(y)|=e^{\int_{y}^{\epsilon}\left(\sum_{j=j_{0}}^{r-1} \Re\left(\lambda_{j}\right) s^{j+n-m} \frac{a_{0}}{b_{0}}(s)-k+\sum_{j=0}^{r-1} \Im\left(\lambda_{j}\right) s^{j}\right) \frac{b_{0}}{s^{2 n-m} a_{0}^{2}+s^{m} b_{0}^{2}}(s) \mathrm{d} s}
\end{aligned}
$$

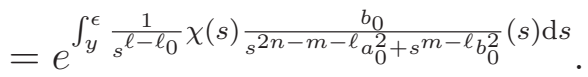

Como $\chi(s) \frac{b_{0}}{s^{2 n-m-\ell} a_{0}^{2}+s^{m-\ell} b_{0}^{2}}(s) \in C^{\infty}((-\epsilon, \epsilon))$ e $\chi(0) \frac{b_{0}}{s^{2 n-m-\ell} a_{0}^{2}+s^{m-\ell} b_{0}^{2}}(0)<0$, supondo $\epsilon>0$ suficientemente pequeno, existe $M_{3}>0$ tal que

$$
|w(y)| \leqslant e^{-M_{3} \int_{y}^{\epsilon} \frac{1}{s^{\ell-\ell_{0}}} \mathrm{~d} s}=e^{\frac{-M_{3}}{\left(\ell-\ell_{0}-1\right)}\left(\frac{1}{y^{\ell-\ell_{0}-1}}-\frac{1}{\epsilon^{\ell-\ell_{0}-1}}\right)} \leqslant 1 .
$$

Logo, concluímos que $w(y)$ é limitada em $I_{\epsilon}^{+}$quando (a2) ocorre.

Agora, suponha que (a3) seja verdadeira. Procedemos como no caso em que (a2) ocorre, para obter $M_{4}>0$ tal que

$$
|w(y)| \geqslant e^{M_{4} \int_{y}^{\epsilon} \frac{1}{s^{\ell-\ell_{0}}} \mathrm{~d} s}=e^{\frac{M_{4}}{\left(\ell-\ell_{0}-1\right)}\left(\frac{1}{y^{\ell-\ell_{0}-1}}-\frac{1}{\epsilon^{\ell-\ell_{0}}-1}\right)} .
$$

Portanto, para todo $j \in\{0,1, \ldots\},\left|y^{j}(y)\right| \rightarrow \infty$ quando $y \rightarrow 0$.

Logo, quando (a3) ocorre, concluímos que não existe $j_{1} \in\{0,1, \ldots\}$ tal que $y^{j_{1}} w(y)$ seja limitada em $I_{\epsilon}^{+}$.

No que segue vamos mostrar que $|v(x)|=O\left(|x|^{j}\right), \forall j \in \mathbb{Z}_{+}$.

Primeiro, vamos analisar os casos nos quais $v$ é dado por (3.56). Estes casos ocorrem, se e somente se, (a3) não ocorre.

Suponha que (a3) não ocorre. Para $x>0$, temos

$$
v(x)=\int_{0}^{x} \frac{w(y)}{w(x)} \frac{h}{a+i b}(y) \mathrm{d} y
$$




$$
=\int_{0}^{x} e^{-i k\left(C_{0+}(x)-C_{0+}(y)\right)+\sum_{j=0}^{r-1} \lambda_{j}\left(C_{j+}(x)-C_{j+}(y)\right)} \frac{h}{a+i b}(y) \mathrm{d} y .
$$

Quando (a1) ocorre, se $\ell-\ell_{0}<1$ então, para $x>0$, encontramos $M_{2} \in \mathbb{Z}_{+}$e $M_{2}^{\prime}$ real positivo de forma que

$$
\begin{aligned}
|v(x)| & \leqslant \int_{0}^{x} e^{\int_{y}^{x}\left[\left(\sum_{j=j_{0}}^{r-1} \Re\left(\lambda_{j}\right) s^{j}\right) \frac{a}{b}(s)-k+\sum_{j=0}^{r-1} \Im\left(\lambda_{j}\right) s^{j}\right] \frac{b}{a^{2}+b^{2}}(s) \mathrm{d} s\left|\frac{h}{a+i b}\right|(y) \mathrm{d} y} \\
& \leqslant \int_{0}^{x} e^{\int_{y}^{x} s^{\ell_{0}-\ell} \chi(s) \frac{b_{0}}{s^{2 n-m-\ell} a_{0}^{2}+s^{m-\ell} b_{0}^{2}}(s) \mathrm{d} s}\left|\frac{h}{a+i b}\right|(y) \mathrm{d} y \\
& \leqslant \int_{0}^{x} e^{M_{2} \int_{y}^{x} s^{\ell_{0}-\ell}(s) \mathrm{d} s}\left|\frac{h}{a+i b}\right|(y) \mathrm{d} y \\
& =\int_{0}^{x} e^{\frac{M_{2}}{\left(\ell_{0}-\ell+1\right)}\left(x^{\ell_{0}-\ell+1}-y^{\ell_{0}-\ell+1}\right)}\left|\frac{h}{a+i b}\right|(y) \mathrm{d} y \\
& \leqslant M_{2}^{\prime} \int_{0}^{x}\left|\frac{h}{a+i b}\right|(y) \mathrm{d} y=O\left(|x|^{j}\right), \forall j \in \mathbb{Z}_{+}
\end{aligned}
$$

se $\ell-\ell_{0}=1$, para $x>0$, temos

$$
\begin{aligned}
|v(x)| & \leqslant \int_{0}^{x} e^{M_{2} \int_{y}^{x} s^{\ell_{0}-\ell}(s) \mathrm{d} s}\left|\frac{h}{a+i b}\right|(y) \mathrm{d} y \\
& =\int_{0}^{x}\left(\frac{x}{y}\right)^{M_{2}}\left|\frac{h}{a+i b}\right|(y) \mathrm{d} y=x^{M_{2}} \int_{0}^{x}\left(\frac{1}{y}\right)^{M_{2}}\left|\frac{h}{a+i b}\right|(y) \mathrm{d} y=O\left(|x|^{j}\right), \forall j \in \mathbb{Z}_{+} .
\end{aligned}
$$

Quando (a2) ocorre, para $x>0$, encontramos $M_{3}$ real positivo de forma que

$$
\begin{aligned}
|v(x)| & \leqslant \int_{0}^{x} e^{\int_{y}^{x}\left[\left(\sum_{j=j_{0}}^{r-1} \Re\left(\lambda_{j}\right) s^{j}\right) \frac{a}{b}(s)-k+\sum_{j=0}^{r-1} \Im\left(\lambda_{j}\right) s^{j}\right] \frac{b}{a^{2}+b^{2}}(s) \mathrm{d} s}\left|\frac{h}{a+i b}\right|(y) \mathrm{d} y \\
& \leqslant \int_{0}^{x} e^{\int_{y}^{x} s^{\ell} \ell^{-\ell} \chi(s) \frac{b_{0}}{s^{2 n-m-\ell} a_{0}^{2}+s^{m-\ell} b_{0}^{2}}(s) \mathrm{d} s}\left|\frac{h}{a+i b}\right|(y) \mathrm{d} y \\
& \leqslant \int_{0}^{x} e^{-M_{3} \int_{y}^{x} \frac{1}{s^{\ell-\ell_{0}}}(s) \mathrm{d} s}\left|\frac{h}{a+i b}\right|(y) \mathrm{d} y \\
& =\int_{0}^{x} e^{\frac{-M_{3}}{\left(\ell-\ell_{0}-1\right)}}\left(\frac{1}{y^{\ell-\ell_{0}-1}-\frac{1}{x^{\ell-\ell_{0}-1}}}\right)\left|\frac{h}{a+i b}\right|(y) \mathrm{d} y \\
& \leqslant \int_{0}^{x}\left|\frac{h}{a+i b}\right|(y) \mathrm{d} y=O\left(|x|^{j}\right), \forall j \in \mathbb{Z}_{+} .
\end{aligned}
$$

Agora, vamos analisar o caso no qual $v$ é dado por (3.57). Este caso ocorre, se e somente se, (a3) ocorre. 
Suponha que (a3) ocorre. Para $x>0$, temos

$$
\begin{aligned}
v(x) & =-\int_{x}^{\epsilon} \frac{w(y)}{w(x)} \frac{h}{a+i b}(y) \mathrm{d} y \\
& =-\int_{x}^{\epsilon} e^{-i k\left(C_{0+}(x)-C_{0+}(y)\right)+\sum_{j=0}^{r-1} \lambda_{j}\left(C_{j+}(x)-C_{j+}(y)\right)} \frac{h}{a+i b}(y) \mathrm{d} y .
\end{aligned}
$$

Portanto, quando (a3) ocorre, para $x>0$, temos

$$
\begin{aligned}
& |v(x)| \leqslant \int_{x}^{\epsilon} e^{\int_{y}^{x}\left[\left(\sum_{j=j_{0}}^{r-1} \Re\left(\lambda_{j}\right) s^{j}\right) \frac{a}{b}(s)+\left(-k+\sum_{j=0}^{r-1} \Im\left(\lambda_{j}\right) s^{j}\right)\right] \frac{b}{a^{2}+b^{2}}(s) \mathrm{d} s}\left|\frac{h}{a+i b}\right|(y) \mathrm{d} y \\
& =\int_{x}^{\epsilon} e^{\int_{y}^{\epsilon}\left(\sum_{j=j_{0}}^{r-1} \Re\left(\lambda_{j}\right) s^{j+n-m} \frac{a_{0}}{b_{0}}(s)-k+\sum_{j=N}^{r-1} \Im\left(\lambda_{j}\right) s^{j}\right) \frac{b_{0}}{s^{2 n-m} a_{0}^{2}+s^{m} b_{0}^{2}}(s) \mathrm{d} s}\left|\frac{h}{a+i b}\right|(y) \mathrm{d} y \\
& =\int_{x}^{\epsilon} e^{-\int_{x}^{y} \frac{1}{s^{\ell-\ell_{0}}} \chi(s) \frac{b_{0}}{s^{2 n-m-\ell} a_{0}^{2}+s^{m-\ell} b_{0}^{2}}(s) \mathrm{d} s}\left|\frac{h}{a+i b}\right|(y) \mathrm{d} y \text {. }
\end{aligned}
$$

Como $\chi(s) \frac{b_{0}}{s^{2 n-m-\ell} a_{0}^{2}+s^{m-\ell} b_{0}^{2}}(s) \in C^{\infty}((-\epsilon, \epsilon))$ e $\chi(0) \frac{b_{0}}{s^{2 n-m-\ell} a_{0}^{2}+s^{m-\ell} b_{0}^{2}}(0)>0$, existe $M_{4}$ real positivo tal que

$$
|v(x)| \leqslant \int_{x}^{\epsilon} e^{-M_{4} \int_{x}^{y} \frac{1}{s^{\ell-\ell_{0}}}(s) \mathrm{d} s}\left|\frac{h}{a+i b}\right|(y) \mathrm{d} y .
$$

Logo, se $0<x<\frac{\epsilon}{2}$, então temos

$$
|v(x)| \leqslant \int_{x}^{2 x} e^{-M_{4} \int_{x}^{y} \frac{1}{s^{\ell-\ell_{0}}}(s) \mathrm{d} s}\left|\frac{h}{a+i b}\right|(y) \mathrm{d} y+\int_{2 x}^{\epsilon} e^{-M_{4} \int_{x}^{y} \frac{1}{s^{\ell-\ell_{0}}}(s) \mathrm{d} s}\left|\frac{h}{a+i b}\right|(y) \mathrm{d} y .
$$

Para $y \geqslant 2 x$, temos

$$
\frac{1}{y^{\ell-\ell_{0}-1}}-\frac{1}{x^{\ell-\ell_{0}-1}} \leqslant \frac{-\left(2^{\ell-\ell_{0}-1}-1\right)}{2^{\ell-\ell_{0}-1}} \frac{1}{x^{\ell-\ell_{0}-1}} .
$$

Portanto,

$$
\begin{aligned}
|v(x)| & \leqslant \int_{x}^{2 x}\left|\frac{h}{a+i b}\right|(y) \mathrm{d} y+\int_{2 x}^{\epsilon} e^{\frac{-M_{4}\left(2^{\ell-\ell_{0}-1}-1\right)}{2^{\ell-\ell_{0}-1}\left(\ell-\ell_{0}-1\right)} \frac{1}{x^{\ell-\ell_{0}-1}}}\left|\frac{h}{a+i b}\right|(y) \mathrm{d} y \\
& \leqslant \int_{x}^{2 x}\left|\frac{h}{a+i b}\right|(y) \mathrm{d} y+M_{4}^{\prime} e^{\frac{-M_{4}\left(2^{\ell-\ell_{0}-1}-1\right)}{2^{\ell-\ell_{0}-1}\left(\ell-\ell_{0}-1\right)} \frac{1}{x^{\ell-\ell_{0}-1}}}=O\left(|x|^{j}\right), \forall j \in \mathbb{Z}_{+} .
\end{aligned}
$$

A análise das soluções e de suas estimativas, na região $x<0$, segue de modo análogo à da região $x>0$. 
Podemos provar estimativas análogas para as derivadas $v^{(\nu)}, \nu \in \mathbb{Z}_{+}$, do qual segue que $v \in C^{\infty}((-\epsilon, \epsilon))$.

Proposição 3.7. Assuma que (3.7) ou (3.8) falha. Defina o inteiro $j_{0}$ como segue:

(i) se $\Re\left(\lambda_{j}\right)=0$ para cada $j \in\{0, \cdots, r-1\}$, seja $j_{0}=r-1$;

(ii) se $\Re\left(\lambda_{j}\right) \neq 0$ para algum $j \in\{0, \cdots, r-1\}$, seja $j_{0}=\min _{0 \leqslant j \leqslant r-1}\left\{j: \Re\left(\lambda_{j}\right) \neq 0\right\}$.

Seja $L$ dado por (3.1). Se $m-n \leqslant j_{0}$, então a equação $L v=\left(\sum_{j=0}^{r-1} \lambda_{j} x^{j}\right) v+h$ tem uma solução $v \in C^{\infty}\left(A_{\epsilon}\right)$, em uma vizinhança de $\Sigma$, para cada $h \in C^{\infty}\left(A_{\epsilon}\right)$ flat em $\Sigma$.

Demonstração. Seja $h \in C^{\infty}\left(A_{\epsilon}\right)$ flat em $\Sigma$. Vamos procurar por $v \in C^{\infty}\left(A_{\epsilon}\right)$ solução de

$$
L v=\left(\sum_{j=0}^{r-1} \lambda_{j} x^{j}\right) v+h
$$

em uma vizinhança de $\Sigma$.

Usando série parcial de Fourier, podemos escrever

$$
v(x, t)=\sum_{k \in \mathbb{Z}} \widehat{v}_{k}(x) e^{i k t} \quad \text { e } \quad h(x, t)=\sum_{k \in \mathbb{Z}} \widehat{h}_{k}(x) e^{i k t} .
$$

Logo, se $v$ é solução de (3.58), temos que $\widehat{v}_{k}$ é uma solução de

$$
i k \widehat{v}_{k}(x)+(a+i b)(x)\left(\widehat{v}_{k}\right)^{\prime}(x)=\left(\sum_{j=0}^{r-1} \lambda_{j} x^{j}\right) \widehat{v}_{k}(x)+\widehat{h}_{k}(x),
$$

para cada $k \in \mathbb{Z}$.

No que segue, para cada $k \in \mathbb{Z}$, vamos procurar por $\widehat{v}_{k} \in C^{\infty}((-\epsilon, \epsilon))$ solução de (3.59) e, então, vamos mostrar que $\sum_{k \in \mathbb{Z}} \widehat{v}_{k}(x) e^{i k t}$ converge na topologia $C^{\infty}$ em uma vizinhança de $\Sigma$.

Considere a equação homogênea, associada a (3.59), dada por

$$
(a+i b)(x)\left(w_{k}\right)^{\prime}(x)=\left(\sum_{j=0}^{r-1} \lambda_{j} x^{j}-i k\right) w_{k}(x) .
$$

Para $x>0$, encontramos

$$
w_{k}(x)=e^{-i k C_{0+}(x)+\sum_{j=0}^{r-1} \lambda_{j} C_{j+}(x)} .
$$


Para $x<0$, encontramos

$$
w_{k}(x)=e^{-i k C_{0-}(x)+\sum_{j=0}^{r-1} \lambda_{j} C_{j-}(x)} .
$$

Portanto, para $x \neq 0$, de (3.59) obtemos

$$
\begin{aligned}
(a+i b)\left(\widehat{v}_{k} w_{k}^{-1}\right)^{\prime} & =\widehat{v}_{k}\left(-\sum_{j=0}^{r-1} \lambda_{j} x^{j}+i k\right) w_{k}^{-1}+w_{k}^{-1}\left[\left(\sum_{j=0}^{r-1} \lambda_{j} x^{j}-i k\right) \widehat{v}_{k}+\widehat{h}_{k}\right] \\
& =w_{k}^{-1} \widehat{h}_{k} .
\end{aligned}
$$

Logo, para $x \neq 0$, temos

$$
\left(\widehat{v}_{k} w_{k}^{-1}\right)^{\prime}=w_{k}^{-1} \frac{\widehat{h}_{k}}{a+i b} .
$$

Agora, vamos nos concentrar na região $x>0$.

Seja $k \in \mathbb{Z}$. Se $k>\Im\left(\lambda_{0}\right)+\Re\left(\lambda_{j_{0}}\right)\left[x^{n-m+j_{0}} \frac{a_{0}(x)}{b_{0}(x)}\right]_{x=0}$, então, podemos proceder como na prova da Proposição 2.3, para concluir que $\left|w_{k}^{-1}\right|$ é limitada em $(0, \epsilon)$ (veja (2.23)).

Logo, para $k>\Im\left(\lambda_{0}\right)+\Re\left(\lambda_{j_{0}}\right)\left[x^{n-m+j_{0}} \frac{a_{0}(x)}{b_{0}(x)}\right]_{x=0}$, encontramos

$$
\widehat{v}_{k}(x)=\int_{0}^{x} \frac{w_{k}(x)}{w_{k}(y)} \frac{\widehat{h}_{k}}{a+i b}(y) \mathrm{d} y
$$

Para $k<\Im\left(\lambda_{0}\right)+\Re\left(\lambda_{j_{0}}\right)\left[x^{n-m+j_{0}} \frac{a_{0}(x)}{b_{0}(x)}\right]_{x=0}$, encontramos

$$
\widehat{v}_{k}(x)=-\int_{x}^{\epsilon} \frac{w_{k}(x)}{w_{k}(y)} \frac{\widehat{h}_{k}}{a+i b}(y) d y .
$$

Para garantir a convergência da série $\sum_{k \in \mathbb{Z}} \widehat{v}_{k}(x) e^{i k t}$ em uma vizinhança de $\Sigma$, na topologia $C^{\infty}$, vamos determinar estimativas para $\left|\widehat{v}_{k}(x)\right|$ utilizando o Lema 3.5.

Faremos isto, supondo inicialmente que $\Im\left(\lambda_{0}\right)+\Re\left(\lambda_{j_{0}}\right)\left[x^{n-m+j_{0}} \frac{a_{0}(x)}{b_{0}(x)}\right]_{x=0} \neq k$ para todo $k \in \mathbb{Z}$.

Para $x>0$ e para cada $k>\Im\left(\lambda_{0}\right)+\Re\left(\lambda_{j_{0}}\right)\left[x^{n-m+j_{0}} \frac{a_{0}(x)}{b_{0}(x)}\right]_{x=0}$, de (3.61) obtemos

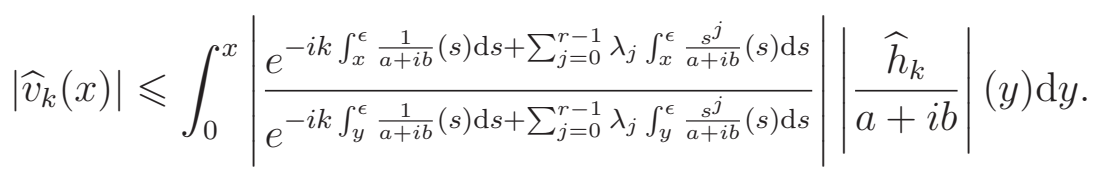


Logo, pelo Lema 3.5, existe $M>0$ e existe $\ell>1$ tais que

$$
\left|\widehat{v}_{k}(x)\right| \leqslant \int_{0}^{x} e^{-\frac{D_{k} M}{(\ell-1)}\left(\frac{1}{x^{\ell-1}}-\frac{1}{y^{\ell-1}}\right)}\left|\frac{\widehat{h}_{k}}{a+i b}\right|(y) \mathrm{d} y=O\left(|x|^{j}\right), \forall j \in \mathbb{Z}_{+},
$$

sendo

$$
D_{k}=\Re\left(\lambda_{j_{0}}\right)\left[x^{n-m+j_{0}} a_{0}(x)\right]_{x=0}+\left(\Im\left(\lambda_{0}\right)-k\right) b_{0}(0) .
$$

Note que $D_{k} M<0$ quando $k>\Im\left(\lambda_{0}\right)+\Re\left(\lambda_{j_{0}}\right)\left[x^{n-m+j_{0}} \frac{a_{0}(x)}{b_{0}(x)}\right]_{x=0}$.

Para $0<x<\frac{\epsilon}{2}$ e para cada $k<\Im\left(\lambda_{0}\right)+\Re\left(\lambda_{j_{0}}\right)\left[x^{n-m+j_{0}} \frac{a_{0}(x)}{b_{0}(x)}\right]_{x=0}$, de (3.62) obtemos

$$
\left|\widehat{v}_{k}(x)\right| \leqslant \int_{x}^{\epsilon}\left|\frac{e^{-i k \int_{x}^{\epsilon} \frac{1}{a+i b}(s) \mathrm{d} s+\sum_{j=0}^{r-1} \lambda_{j} \int_{x}^{\epsilon} \frac{s^{j}}{a+i b}(s) \mathrm{d} s}}{e^{-i k \int_{y}^{\epsilon} \frac{1}{a+i b}(s) \mathrm{d} s+\sum_{j=0}^{r-1} \lambda_{j} \int_{y}^{\epsilon} \frac{s^{j}}{a+i b}(s) \mathrm{d} s}}\right|\left|\frac{\widehat{h}_{k}}{a+i b}\right|(y) \mathrm{d} y .
$$

Logo, pelo Lema 3.5 , existe $M>0$ e existe $\ell>1$ tais que

$$
\begin{aligned}
\left|\widehat{v}_{k}(x)\right| & \leqslant \int_{x}^{\epsilon} e^{\frac{D_{k} M}{(\ell-1)}\left(\frac{1}{y^{\ell-1}}-\frac{1}{x^{\ell-1}}\right)}\left|\frac{\widehat{h}_{k}}{a+i b}\right|(y) \mathrm{d} y \\
& \leqslant \int_{x}^{2 x}\left|\frac{\widehat{h}_{k}}{a+i b}\right|(y) \mathrm{d} y+\int_{2 x}^{\epsilon} e^{\frac{D_{k} M}{(\ell-1)}\left(\frac{1}{y^{\ell-1}}-\frac{1}{x^{\ell-1}}\right)}\left|\frac{\widehat{h}_{k}}{a+i b}\right|(y) \mathrm{d} y,
\end{aligned}
$$

já que $D_{k} M>0$ quando $k<\Im\left(\lambda_{0}\right)+\Re\left(\lambda_{j_{0}}\right)\left[x^{n-m+j_{0}} \frac{a_{0}(x)}{b_{0}(x)}\right]_{x=0}$.

Para $y \geqslant 2 x$, temos

$$
\frac{1}{y^{\ell-1}}-\frac{1}{x^{\ell-1}} \leqslant \frac{-\left(2^{\ell-1}-1\right)}{2^{\ell-1}} \frac{1}{x^{\ell-1}}
$$

Portanto,

$$
\left|\widehat{v}_{k}(x)\right| \leqslant \int_{x}^{2 x}\left|\frac{\widehat{h}_{k}}{a+i b}\right|(y) \mathrm{d} y+M^{\prime} e^{\frac{-D_{k} M\left(2^{\ell-1}-1\right)}{2^{\ell-1}(\ell-1)} \frac{1}{x^{\ell-1}}}=O\left(|x|^{j}\right), \forall j \in \mathbb{Z}_{+} .
$$

Agora, vamos supor que $\Im\left(\lambda_{0}\right)+\Re\left(\lambda_{j_{0}}\right)\left[x^{n-m+j_{0}} \frac{a_{0}(x)}{b_{0}(x)}\right]_{x=0}=k_{1}$ para algum $k_{1} \in \mathbb{Z}$. Neste caso procedemos como anteriormente para obter $\widehat{v}_{k}$ e estimativas para $\left|\widehat{v}_{k}\right|$ quando $k \neq k_{1}$. Agora, aplicando o Lema 3.6 obtemos $\widehat{v}_{k_{1}}(x) \in C^{\infty}((-\epsilon, \epsilon))$ tal que

$$
i k_{1} \widehat{v}_{k_{1}}(x)+(a+i b)(x)\left(\widehat{v}_{k_{1}}\right)^{\prime}(x)=\left(\sum_{j=0}^{r-1} \lambda_{j} x^{j}\right) \widehat{v}_{k_{1}}(x)+\widehat{h}_{k_{1}}(x) .
$$


Para finalizar a análise das soluções e de suas estimativas, devemos observar que o processo utilizado na região $x>0$, procede de modo análogo para a região $x<0$.

Em todos os caso, podemos provar estimativas análogas para as derivadas $\widehat{v}_{k}^{(\nu)}, \nu \in \mathbb{Z}_{+}$, do qual segue que para cada $k \in \mathbb{Z}$, a função $\widehat{v}_{k}$ e suas derivadas são rapidamente decrescentes quando $|k| \rightarrow \infty$. Portanto, a sequência $\left(\widehat{v}_{k}\right)$ define uma função suave $v$, a qual é solução de $L v=\left(\sum_{j=0}^{r-1} \lambda_{j} x^{j}\right) v+h$ em uma vizinhança de $\Sigma$.

\subsection{Não existência de solução}

Nesta seção apresentamos condições sobre $p$ e $f$, sob as quais não é possível obter solução $C^{\infty}$ da equação

$$
\mathcal{L}_{p} u=f
$$

em uma vizinhança de $\Sigma$, sendo $\mathcal{L}_{p}$ definido em $(3.2)$ e $p, f \in C^{\infty}\left(A_{\epsilon}\right)$.

Pela afirmação feita na página 39 , o estudo da resolubilidade de $\mathcal{L}_{p} u=f$ em uma vizinhança de $\Sigma$ é reduzido ao caso em que $f$ é flat em $\Sigma$. Logo, usando a Proposição 3.4 reduzimos o estudo da resolubilidade da equação $\mathcal{L}_{p} u=f$ em uma vizinhança de $\Sigma$ ao estudo da resolubilidade da equação

$$
L u=\left(\sum_{j=0}^{r-1} \lambda_{j} x^{j}\right) u+f
$$

em uma vizinhança de $\Sigma$, com $f$ flat em $\Sigma$.

Na seção 3.1 analisamos os casos em que $m-n \leqslant j_{0}=\min _{0 \leqslant j \leqslant r-1}\left\{j: \Re\left(\lambda_{j}\right) \neq 0\right\}$, então resta-nos supor que $m-n>j_{0}$.

Note que:

- $m \leqslant n \Longrightarrow m-n \leqslant j_{0}$.

- $n<m<2 n-1 \Longrightarrow r=n$ e então $m-n<n-1$; logo se $j_{0}=n-1$ então $m-n<j_{0}$.

Portanto nesta seção vamos supor que

- $n<m<2 n-1$;

- $j_{0} \leqslant n-2(=r-2)$; em particular, $N \leqslant n-2$, pois $N \leqslant j_{0}$.

- $m-n>j_{0}$ 
Iremos verificar que sob as hipóteses acima existe $f \in C^{\infty}\left(A_{\epsilon}\right)$ flat em $\Sigma$ para a qual não existe $u \in C^{\infty}\left(A_{\epsilon}\right)$ solução de $L u=\left(\sum_{j=0}^{r-1} \lambda_{j} x^{j}\right) u+f$.

Proposição 3.8. Seja $\mathcal{L}_{p}$ dado por (3.2) e sejam $\lambda_{1}, \ldots, \lambda_{n-1}$ dados por (3.4). Assuma que $n<m<2 n-1$. Seja $\mathcal{F} \subset C^{\infty}\left(A_{\epsilon}\right)$ definido por:

(1) se $\lambda_{0} \notin i \mathbb{Z}$ definamos $\mathcal{F}=C^{\infty}\left(A_{\epsilon}\right)$;

(2) se $\lambda_{0} \in i \mathbb{Z}$ definamos $\mathcal{F}$ o subespaço de $C^{\infty}\left(A_{\epsilon}\right)$ de todas as funções $f$ satisfazendo (3.13), sendo $N$ o menor inteiro satisfazendo $1 \leqslant N \leqslant n-2$, tal que $\lambda_{N} \neq 0$.

Se, para cada $f \in \mathcal{F}, \mathcal{L}_{p} u=f$ tem solução $u \in C^{\infty}\left(A_{\epsilon}\right)$ em uma vizinhança de $\Sigma$, então existe $\delta_{0}>0$ tal que $\mathcal{L}_{p} w=f$ tem uma solução $u \in C^{\infty}\left(A_{\epsilon}\right)$, em uma vizinhança de $\left[-\delta_{0}, \delta_{0}\right] \times S^{1}$, para cada $f \in \mathcal{F}$.

Demonstração. Se $\lambda_{0} \in i \mathbb{Z}$ então defina

$$
Q_{N-1}(x, t) \doteq \sum_{j=0}^{N-1} \frac{1}{j !}\left(\int_{0}^{t} \frac{\partial^{j} p}{\partial x^{j}}(0, \sigma) d \sigma\right) x^{j}
$$

Para cada $j \in\{0,1, \ldots\}$, considere a distribuição $1 \otimes \delta^{(j)} \in \mathcal{E}^{\prime}\left(A_{\epsilon}\right)$, dada por

$$
\left\langle 1 \otimes \delta^{(j)}, f\right\rangle \doteq \int_{0}^{2 \pi} \frac{\partial^{j} f}{\partial x^{j}}(0, \sigma) d \sigma, f \in C^{\infty}\left(A_{\epsilon}\right)
$$

Defina

$$
S \doteq \begin{cases}\{0\}, & \text { se } \lambda_{0} \notin i \mathbb{Z}, \\ \operatorname{span}\left\langle e^{-Q_{N-1}}\left(1 \otimes \delta^{(j)}\right), j=0, \ldots, N-1\right\rangle, & \text { se } \lambda_{0} \in i \mathbb{Z} .\end{cases}
$$

e

$$
S^{\circ} \doteq\left\{f \in C^{\infty}\left(A_{\epsilon}\right) ;\langle u, f\rangle=0, \forall u \in S\right\}
$$

Note $S^{\circ} \subset C^{\infty}\left(A_{\epsilon}\right)$ é um subespaço vetorial fechado, pois $S^{\circ}=\bigcap_{u \in S} u^{-1}(0)$. Portanto, $S^{\circ}$ é um subespaço vetorial fechado do espaço de Fréchet $C^{\infty}\left(A_{\epsilon}\right)$; consequentemente, também é um espaço de Fréchet (veja, por exemplo, página 85 de [13]).

Suponha que para cada $f \in \mathcal{F}, \mathcal{L}_{p} w=f$ tem solução $u \in C^{\infty}\left(A_{\epsilon}\right)$ em alguma vizinhança de $\Sigma$, vamos repetir os argumentos usados na página 794 de [1] (veja, também, e [6], [2]e [3]); isto é, usando o Teorema de Baire e o Teorema da Aplicação Aberta provaremos que existe $\delta_{0}>0$ tal que, dada $f \in S^{\circ}$, existe $w \in C^{\infty}\left(A_{\epsilon}\right)$ solução de $\mathcal{L}_{p} w=f$ em alguma vizinhança de $\left[-\delta_{0}, \delta_{0}\right] \times S^{1}$. 
Para cada $\ell \in \mathbb{Z}_{+}$, defina

$$
F_{\ell}=\left\{(f, u) \in S^{\circ} \times C^{\infty}\left(\overline{\mathcal{U}}_{\ell}\right) ; \mathcal{L}_{p} u=f \text { em } \mathcal{U}_{\ell}\right\}
$$

sendo $\mathcal{U}_{\ell}=\left(-\delta_{\ell}, \delta_{\ell}\right) \times S^{1}$ e $\delta_{\ell} \searrow 0, \operatorname{com} \delta_{1}=\epsilon / 2$.

Note que, para cada $\ell \in \mathbb{Z}_{+}, F_{\ell}$ é um espaço de Fréchet. De fato, como o operador $\mathcal{L}_{p}$ é linear e contínuo na topologia $C^{\infty}, F_{\ell}$ é um subespaço vetorial fechado do espaço de Fréchet $S^{\circ} \times C^{\infty}\left(\overline{\mathcal{U}}_{\ell}\right)$.

Defina

$$
\begin{aligned}
\pi_{\ell}: \quad F_{\ell} & \rightarrow S^{\circ} \\
(f, u) & \mapsto f
\end{aligned}
$$

a projeção sobre o primeiro fator.

Afirmamos que

$$
\bigcup_{\ell} \pi_{\ell}\left(F_{\ell}\right)=S^{\circ}
$$

De fato, a inclusão $\bigcup_{\ell} \pi_{\ell}\left(F_{\ell}\right) \subset S^{\circ}$ segue da definição de $F_{\ell}$ e de $\pi_{\ell}$. Para obter a inclusão $S^{\circ} \subset \bigcup_{\ell} \pi_{\ell}\left(F_{\ell}\right)$ observe que, sob nossas hipóteses, para cada $f \in S^{\circ}$ existem $\epsilon_{0}>0$ e $u \in C^{\infty}$ solução de $\mathcal{L}_{p} u=f$ na vizinhança $\mathcal{U}_{0}=\left(-\epsilon_{0}, \epsilon_{0}\right) \times S^{1} \supset \overline{\mathcal{U}}_{\ell_{1}}$, para algum $\ell_{1} \in \mathbb{Z}_{+}$, já que $S^{\circ}=\mathcal{F}$. Portanto $f=\pi_{\ell_{1}}(f, u) \in \pi_{\ell_{1}}\left(F_{\ell_{1}}\right) \subset \bigcup_{\ell} \pi_{\ell}\left(F_{\ell}\right)$.

Como a projeção é contínua, para cada $\ell \in \mathbb{Z}_{+}, \pi_{\ell}\left(F_{\ell}\right)$ é fechado no espaço de Fréchet $S^{\circ}$. Portanto, pelo Teorema de Baire (veja, por exemplo, página 42 de [11]), $\operatorname{int}\left(\pi_{\ell_{0}}\left(F_{\ell_{0}}\right)\right) \neq \emptyset$ para algum $\ell_{0} \in \mathbb{Z}_{+}$.

Assim, pelo Teorema da Aplicação Aberta (veja, por exemplo, página 47 de [11]), concluímos que $\pi_{\ell_{0}}\left(F_{\ell_{0}}\right)=S^{\circ}$. Isto significa que, para $\delta_{0} \doteq \delta_{\ell_{0}}$, tem-se

$$
\mathcal{L}_{p}\left(C^{\infty}\left(\left[-\delta_{0}, \delta_{0}\right] \times S^{1}\right)\right) \supset\left(S^{\circ} \cap C^{\infty}\left(\left[-\delta_{0}, \delta_{0}\right] \times S^{1}\right)\right)
$$

Por (3.63), concluímos que $\mathcal{L}_{p} w=f$ tem uma solução $u \in C^{\infty}\left(A_{\epsilon}\right)$, em uma vizinhança de $\left[-\delta_{0}, \delta_{0}\right] \times S^{1}$, para cada $f \in \mathcal{F}$.

Lema 3.9. Seja $\mathcal{L}_{p}$ dado por (3.2) e sejam $\lambda_{1}, \ldots, \lambda_{n-1}$ dados por (3.4). Assuma que $n<m<2 n-1$ e que $\Re\left(\lambda_{j}\right) \neq 0$ para algum $j \in\{0, \cdots, n-2\}$ e, além disso, $m-n>j_{0}=\min _{0 \leq j \leq n-2}\left\{j: \Re\left(\lambda_{j}\right) \neq 0\right\}$. Se, para cada $g \in C^{\infty}\left(A_{\epsilon}\right)$ e flat em $\Sigma$, $L u=\left(\sum_{j=0}^{n-1} \lambda_{j} x^{j}\right) u+g$ tem solução $C^{\infty}$ em uma vizinhança de $\Sigma$, então existe $\delta_{0}>0$ tal que $L w=\left(\sum_{j=0}^{n-1} \lambda_{j} x^{j}\right) w+g$ tem solução $w \in C^{\infty}\left(A_{\epsilon}\right)$, em uma vizinhança de $\left[-\delta_{0}, \delta_{0}\right] \times S^{1}$, para cada $g \in C^{\infty}\left(A_{\epsilon}\right)$ e flat em $\Sigma$. 
Demonstração. Por hipótese, para cada $g \in C^{\infty}\left(A_{\epsilon}\right)$, flat em $\Sigma$, existe $v \in C^{\infty}\left(A_{\epsilon}\right)$ tal que

$$
L u=\left(\sum_{j=0}^{n-1} \lambda_{j} x^{j}\right) u+g
$$

em uma vizinhança de $\Sigma$.

Daí, pela Proposição 3.4, para cada $f \in C^{\infty}\left(A_{\epsilon}\right)$, flat em $\Sigma$, existe $u \in C^{\infty}\left(A_{\epsilon}\right)$ tal que

$$
\mathcal{L}_{p} u=f
$$

em uma vizinhança de $\Sigma$.

Consequentemente, pela Proposição 3.3 e 3.8, temos que para cada $f \in C^{\infty}\left(A_{\epsilon}\right)$, flat em $\Sigma$, existe $u \in C^{\infty}\left(A_{\epsilon}\right)$ tal que

$$
\mathcal{L}_{p} w=f
$$

em uma vizinhança de $\left[-\delta_{0}, \delta_{0}\right] \times S^{1}$.

Portanto, segue da demonstração da Proposição 3.4 que para cada $g \in C^{\infty}\left(A_{\epsilon}\right)$, flat em $\Sigma$, existe $v \in C^{\infty}\left(A_{\epsilon}\right)$ solução de

$$
L v=\left(\sum_{j=0}^{n-1} \lambda_{j} x^{j}\right) v+g
$$

em uma vizinhança de $\left[-\delta_{0}, \delta_{0}\right] \times S^{1}$.

Lema 3.10. Seja $\delta_{0}>0$ dado pelo Lema 3.9 e seja $x_{0} \in\left(0, \delta_{0}\right)$. Para cada $k \in \mathbb{Z}$, seja

$$
g_{k}:(-\epsilon, \epsilon) \rightarrow \mathbb{C}
$$

definida por

$$
g_{k}(x)=\varphi_{1}(x) \cdot(a+i b)(x) \cdot c_{k} \cdot \varphi_{2}(x)
$$

sendo $\left(c_{k}\right)$ uma sequência de números reais positivos rapidamente decrescente, $\varphi_{1}:(-\epsilon, \epsilon) \rightarrow \mathbb{R}$ dada por

$$
\varphi_{1}(x)= \begin{cases}e^{-\frac{1}{x}}, & x>0 \\ 0, & x \leqslant 0\end{cases}
$$

e $\varphi_{2}:(-\epsilon, \epsilon) \rightarrow \mathbb{C}$ dada por

$$
\varphi_{2}(x)=\left\{\begin{array}{ll}
e^{-i\left(\int_{x}^{x_{0}}\left(-k+\sum_{j=0}^{n-1} \Im\left(\lambda_{j}\right) s_{j} s^{j}\right) \frac{a}{a^{2}+b^{2}}(s) \mathrm{d} s-\int_{x}^{x_{0}}\left(\sum_{j=j_{0}}^{n-1} \Re\left(\lambda_{j}\right) s^{j}\right) \frac{b}{a^{2}+b^{2}}(s) \mathrm{d} s\right)}, & x>0 \\
0, & x \leqslant 0
\end{array} .\right.
$$


A sequência $\left(g_{k}\right)$ define uma função $g \in C^{\infty}\left(A_{\epsilon}\right)$ e flat em $\Sigma$.

Demonstração. Note que $g_{k}$ é de classe $C^{\infty}$ para cada $x \neq 0$.

Agora, considere

$$
\varphi_{3}(x)=\varphi_{1}(x)(a+i b)(x)
$$

Como $\varphi_{3}(x)$ é flat em $x=0$, temos que para qualquer função $q(x)$ que se anula de ordem finita em $x=0$ vale:

$$
\frac{\varphi_{3}^{(j)}}{q}(0)=0, \quad \forall j \in \mathbb{Z}_{+}
$$

Assim, para qualquer $s \leqslant j$ temos $\left(\varphi_{3}^{(s)} \varphi_{2}^{(j-s)}\right)\left(0^{ \pm}\right)=0$.

De fato, existe uma função $q$ que se anula de ordem finita em $x=0$ tal que $q \varphi_{2}^{(j-s)}=$ $\varphi_{2} \in C^{\infty}\left(0, x_{0}\right) \cap L^{\infty}\left[0, x_{0}\right]$, além disso, $\varphi_{2}(x) \equiv 0$ e $\varphi_{3}(x) \equiv 0$ em $(-\epsilon, 0)$.

Logo,

$$
g_{k}^{(j)}\left(0^{ \pm}\right)=c_{k} \sum_{s \leq j}\left(\begin{array}{c}
j \\
s
\end{array}\right)\left(\varphi_{3}^{(s)} \varphi_{2}^{(j-s)}\right)\left(0^{ \pm}\right)=0, \quad \text { para cada } j
$$

daí $g_{k}$ é de classe $C^{\infty}$ e flat em $x=0$. Além disso, para cada $j$ fixado,

$$
\left|\widehat{g}_{k}^{(j)}(x)\right| \leq \sum_{s \leq j}\left(\begin{array}{c}
j \\
s
\end{array}\right)\left|\varphi_{1}^{(s)} \varphi_{2}^{(j-s)}(x) c_{k}\right| \leq \sum_{s \leq j} C_{j s}|| \varphi_{1}^{(s)} q_{j-s}||_{\infty}|k|^{j-s} c_{k} \leq C_{j}|k|{ }^{j} c_{k},
$$

sendo $C_{j}=\max _{s \leq j}\left\{C_{j s}\left\|\varphi_{1}^{(s)} q_{j-s}\right\|_{\infty}\right\}$, e $q_{j-s}$ somatório de derivadas de potências das funções $\frac{a}{a^{2}+b^{2}}(x)$ e $\frac{b}{a^{2}+b^{2}}(x)$. Porém, de $\left(c_{k}\right)$ rapidamente decrescente, temos que dados $\ell, j \in \mathbb{N}$, existe $C^{\prime}>0$ tal que $\left|c_{k}\right| \leq \frac{C^{\prime}}{(1+|k|)^{\ell+j}}, \forall k$, e, portanto,

$$
\left|\widehat{g}_{k}^{(j)}(x)\right| \leq \frac{C_{j}^{\prime}}{(1+|k|)^{\ell}}, \forall k
$$

Desse modo, a função $g$ definida por

$$
g(x, t) \doteq \sum_{k \in \mathbb{Z}} g_{k}(x) e^{i k t}
$$

é de classe $C^{\infty}$ em $A_{\epsilon}$, e flat em $\Sigma$. 
No lema técnico a seguir, destacamos estimativas que são obtidas procedendo como na prova da Proposição 2.4.

Lema 3.11. Suponha que $\Re\left(\lambda_{j}\right) \neq 0$ para algum $j \in\{0, \cdots, n-2\}$ e, além disso, $m-n>j_{0}=\min _{0 \leq j \leq n-2}\left\{j: \Re\left(\lambda_{j}\right) \neq 0\right\}$. Existem $\epsilon_{k}>0$ e $\varphi_{k} \in C^{\infty}((-\epsilon, \epsilon))$ tais que

$$
\begin{gathered}
\left|e^{i k C_{0+}(x)-\sum_{j=0}^{n-1} \lambda_{j} C_{j+}(x)}\right|=e^{\frac{\Re\left(\lambda_{j_{0}}\right)}{a_{0}(0)} \int_{x}^{\epsilon} \frac{1}{s^{n-j_{0}}} \varphi_{k}(s) \mathrm{d} s}, \text { para todo } x \in(0, \epsilon), \\
\left|e^{i k C_{0-}(x)-\sum_{j=0}^{n-1} \lambda_{j} C_{j-}(x)}\right|=e^{\frac{-\Re\left(\lambda_{j_{0}}\right)}{a_{0}(0)} \int_{-\epsilon}^{x} \frac{1}{s^{n-j_{0}}} \varphi_{k}(s) \mathrm{d} s}, \text { para todo } x \in(-\epsilon, 0),
\end{gathered}
$$

$e$

$$
\frac{1}{2} \leq \varphi_{k}(s) \leq \frac{3}{2}, \quad \text { para todo } \quad s \in\left(-\epsilon_{k}, \epsilon_{k}\right)
$$

Demonstração. Sob nossas hipóteses, para $x \neq 0$, podemos escrever

$$
\Re\left(k i C_{0 \pm}(x)-\sum_{j=0}^{n-1} \lambda_{j} C_{j \pm}(x)\right)=\frac{\Re\left(\lambda_{j_{0}}\right)}{a_{0}(0)} \int_{x}^{ \pm \epsilon} \frac{1}{y^{n-j_{0}}} \varphi_{k}(y) \mathrm{d} y
$$

sendo

$$
\begin{aligned}
\varphi_{k}(y)=y^{n-j_{0}} \frac{a_{0}(0)}{\Re\left(\lambda_{j_{0}}\right)}\left\{\left(\sum_{j=j_{0}}^{n-1} \Re\left(\lambda_{j}\right) y^{j}\right)\right. & \frac{a}{a^{2}+b^{2}}(y) \\
+ & {\left.\left[\left(-k+\Im\left(\lambda_{0}\right)\right)+\sum_{j=1}^{n-1} \Im\left(\lambda_{j}\right) y^{j}\right] \frac{b}{a^{2}+b^{2}}(y)\right\} . }
\end{aligned}
$$

Note que $\varphi_{k} \in C^{\infty}((-\epsilon, \epsilon))$ e $\varphi_{k}(0)=1$; além disso, podemos encontrar $\epsilon_{k}>0$ tal que

$$
\frac{1}{2} \leq \varphi_{k}(x) \leq \frac{3}{2}, \quad \text { se } \quad x \in\left(-\epsilon_{k}, \epsilon_{k}\right)
$$

Concluímos a demonstração observando que (3.64) e (3.65) seguem de (3.66).

Lema 3.12. Seja $\delta_{0}$ dado pelo Lema 3.9. Para cada $k \in \mathbb{Z}$, seja $g_{k}$ dado pelo Lema 3.10. Suponha que $\Re\left(\lambda_{j}\right) \neq 0$ para algum $j \in\{0, \cdots, n-2\}$. Seja $j_{0}=\min _{0 \leqslant j \leqslant n-2}\left\{j: \Re\left(\lambda_{j}\right) \neq 0\right\}$. Se $\Re\left(\lambda_{j_{0}}\right) a_{0}(0)<0$ e $m-n>j_{0}$, então as soluções de classe $C^{\infty}$ de

$$
i k v_{k}(x)+(a+i b)(x)\left(v_{k}\right)^{\prime}(x)=\left(\sum_{j=0}^{n-1} \lambda_{j} x^{j}\right) v_{k}+g_{k}(x)
$$


em uma vizinhança de $\left[-\delta_{0}, \delta_{0}\right]$, são da forma

$$
v_{k}(x)=\left\{\begin{array}{ll}
e^{-i k C_{0+}(x)+\sum_{j=0}^{n-1} \lambda_{j} C_{j+}(x)} \int_{0}^{x} e^{i k C_{0+}(y)-\sum_{j=0}^{n-1} \lambda_{j} C_{j+}(y)} \frac{\widehat{g}_{k}}{a+i b}(y) \mathrm{d} y, & 0<x \leqslant \delta_{0} \\
0, & x=0 \\
H_{k} e^{-k i C_{0-}(x)+\sum_{j=0}^{n-1} \lambda_{j} C_{j-}(x)}, & -\delta_{0} \leqslant x<0
\end{array},\right.
$$

sendo $H_{k}$ uma constante que deve ser nula quando $n-j_{0}$ for impar.

Demonstração. Seja $v_{k} \in C^{\infty}((-\epsilon, \epsilon))$ solução de $(3.67)$ em uma vizinhança de $\left[-\delta_{0}, \delta_{0}\right]$. Então, $v_{k}$ tem a forma $v_{k}=u_{k}+h_{k}(x)$, sendo $h_{k}$ uma solução arbitrária da equação homogênea

$$
i k h_{k}(x)+(a+i b)(x)\left(h_{k}\right)^{\prime}(x)=\left(\sum_{j=0}^{n-1} \lambda_{j} x^{j}\right) h_{k}(x),
$$

e $u_{k}$ uma solução particular da equação não homogênea (3.67).

Afirmamos que $e^{i k C_{0+}(y)-\sum_{j=0}^{n-1} \lambda_{j} C_{j+}(y)}$ é limitada em $(0, \epsilon)$. De fato, pelo Lema 3.11, existem $\epsilon_{k}>0$ e $\varphi_{k} \in C^{\infty}((-\epsilon, \epsilon))$ tais que, para $0<y<\epsilon_{k}$, obtemos

$$
\begin{aligned}
\left|e^{i k C_{0+}(y)-\sum_{j=0}^{n-1} \lambda_{j} C_{j+}(y)}\right| & =e^{\frac{\Re\left(\lambda_{j_{0}}\right)}{a_{0}(0)} \int_{y}^{\epsilon} \frac{1}{s^{n-j_{0}}} \varphi_{k}(s) \mathrm{d} s} e^{\frac{\Re\left(\lambda_{j_{0}}\right)}{a_{0}(0)} \int_{\epsilon_{k}}^{\epsilon} \frac{1}{s^{n-j_{0}}} \varphi_{k}(s) \mathrm{d} s} \\
& \leqslant M_{k} e^{\frac{\Re\left(\lambda_{j_{0}}\right)}{a_{0}(0)} \frac{1}{2} \int_{y}^{\epsilon} \frac{1}{s^{n-j_{0}}} \mathrm{~d} s}=M_{k} e^{\left.\frac{\Re\left(\lambda_{j_{0}}\right)}{2 a_{0}(0)} \frac{-1}{\left(n-j_{0}-1\right)} \frac{1}{s^{n-j_{0}-1}}\right|_{y} ^{\epsilon_{k}}} \\
& =M_{k} e^{\frac{\Re\left(\lambda_{j_{0}}\right)}{2 a_{0}(0)\left(n-j_{0}-1\right)}\left(\frac{1}{y^{n-j_{0}-1}}-\frac{1}{\epsilon_{k}^{n-j_{0}-1}}\right)},
\end{aligned}
$$

sendo $M_{k}=e^{\frac{\Re\left(\lambda_{j_{0}}\right)}{a_{0}(0)} \int_{\epsilon_{k}}^{\epsilon} \frac{1}{s^{n-j_{0}}} \varphi_{k}(s) \mathrm{d} s}$.

Assim, para $0<y<\epsilon_{k}$, temos

$$
\left|e^{i k C_{0+}(y)-\sum_{j=0}^{n-1} \lambda_{j} C_{j+}(y)}\right| \leqslant M_{k}^{\prime} e^{\frac{\Re\left(\lambda_{j}\right)}{2 a_{0}(0)\left(n-j_{0}-1\right)} \frac{1}{y^{n-j_{0}-1}}}
$$

sendo $M_{k}^{\prime}=M_{k} e^{-\frac{\Re\left(\lambda_{0}\right)}{2 a_{0}(0)\left(n-j_{0}-1\right)} \frac{1}{\epsilon_{k}^{n-j_{0}-1}}}$; note que $n-j_{0}>2$, conforme verificamos na prova do Lema 2.4 (ver página 16).

Como $\frac{\Re\left(\lambda_{j_{0}}\right)}{a_{0}(0)}<0$, concluímos que $e^{i k C_{0+}(y)-\sum_{j=0}^{n-1} \lambda_{j} C_{j+}(y)}$ é limitada em $(0, \epsilon)$, para cada $k \in \mathbb{Z}$; consequentemente $e^{i k C_{0+}(y)-\sum_{j=0}^{n-1} \lambda_{j} C_{j+}(y)}$ é integrável em [0, $\left.\delta_{0}\right]$.

Logo, para $x>0$, encontramos

$$
u_{k}(x)=e^{-i k C_{0+}(x)+\sum_{j=0}^{n-1} \lambda_{j} C_{j+}(x)} \int_{0}^{x} e^{i k C_{0+}(y)-\sum_{j=0}^{n-1} \lambda_{j} C_{j+}(y)} \frac{g_{k}}{a+i b}(y) \mathrm{d} y .
$$


Afirmamos que,

$$
\left|u_{k}(x)\right|=O\left(|x|^{j}\right), \forall j \in \mathbb{Z}_{+} .
$$

De fato. Para $0<y<x<\epsilon_{k}$, de (3.64) temos

$$
\left|\frac{e^{i k C_{0+}(y)-\sum_{j=0}^{n-1} \lambda_{j} C_{j+}(y)}}{e^{i k C_{0+}(x)-\sum_{j=0}^{n-1} \lambda_{j} C_{j+}(x)}}\right|=e^{\frac{\Re\left(\lambda_{j_{0}}\right)}{a_{0}(0)} \int_{y}^{x} \frac{1}{s^{n-j_{0}}} \varphi_{k}(s) \mathrm{d} s} \leqslant e^{\frac{\Re\left(\lambda_{j_{0}}\right)}{2 a_{0}(0)} \int_{y}^{x} \frac{1}{s^{n-j_{0}}} \mathrm{~d} s} \leqslant 1
$$

consequentemente, para $0<x<\epsilon_{k}$, temos

$$
\begin{aligned}
\left|u_{k}(x)\right| \leqslant \int_{0}^{x}\left|\frac{e^{i k C_{0+}(y)-\sum_{j=0}^{n-1} \lambda_{j} C_{j+}(y)}}{e^{i k C_{0+}(x)-\sum_{j=0}^{n-1} \lambda_{j} C_{j+}(x)}} \frac{\widehat{g}_{k}}{a+i b}\right| & (y) \mathrm{d} y \\
& \leqslant \int_{0}^{x}\left|\frac{g_{k}}{a+i b}\right|(y) \mathrm{d} y=O\left(|x|^{j}\right), \forall j \in \mathbb{Z}_{+} .
\end{aligned}
$$

Note que estimativas análogas podem ser provadas para as derivadas $u_{k}^{(\nu)}, \nu \in \mathbb{Z}_{+}$, na região $x>0$.

Concluímos que $u_{k}$ definida por

$$
u_{k}(x) \doteq\left\{\begin{array}{ll}
e^{-i k C_{0+}(x)+\sum_{j=0}^{n-1} \lambda_{j} C_{j+}(x)} \int_{0}^{x} e^{i k C_{0+}(y)-\sum_{j=0}^{n-1} \lambda_{j} C_{j+}(y)} \frac{g_{k}}{a+i b}(y) \mathrm{d} y, & x>0 \\
0, & x \leqslant 0
\end{array},\right.
$$

é uma solução particular, de classe $C^{\infty}$, da equação não homogênea (3.67).

Agora, vamos analisar a solução $h_{k}$ da equação homogênea (3.68). Simples cálculos mostram que a solução da equação homogênea é dada por

$$
h_{k}(x)=h_{k}(\epsilon) e^{-i k C_{0+}(x)+\sum_{j=0}^{n-1} \lambda_{j} C_{j+}(x)} \quad \text { se } \quad 0<x<\epsilon,
$$

e

$$
h_{k}(x)=h_{k}(-\epsilon) e^{i k C_{0-}(x)+\sum_{j=0}^{n-1} \lambda_{j} C_{j-}(x)}, \quad \text { se } \quad-\epsilon<x<0 .
$$

Afirmamos que $h_{k} \notin C^{\infty}$ se $h_{k}(\epsilon) \neq 0$.

De fato, para $0<x<\epsilon_{k}$, por (3.69) temos

$$
\left|h_{k}(x)\right|=\frac{\left|h_{k}(\epsilon)\right|}{\left|e^{i k C_{0-}(x)-\sum_{j=N}^{n-1} \lambda_{j} C_{j-}(x)}\right|} \geqslant \frac{\left|h_{k}(\epsilon)\right|}{M_{k}^{\prime}} e^{\frac{-\Re\left(\lambda_{j_{0}}\right)}{2 a_{0}(0)\left(n-j_{0}-1\right)} \frac{1}{x^{n-j_{0}-1}}} .
$$

Logo, $h_{k}$ não é contínua na origem se $h_{k}(\epsilon) \neq 0$, pois $n-j_{0}>1$ e $\frac{\Re\left(\lambda_{j_{0}}\right)}{a_{0}(0)}<0$. Portanto, da afirmação acima e da prova da Proposição 2.4, concluímos que 
- para $n-j_{0}$ ímpar, $h_{k}(x) \in C^{\infty}$ se, e somente se, $h_{k}(\epsilon)=0=h_{k}(-\epsilon)$;

- para $n-j_{0}$ par, $h_{k}(x) \in C^{\infty}$ se, e somente se, $h_{k}(\epsilon)=0$.

Assim, concluímos que as soluções de classe $C^{\infty}$, em uma vizinhança de $\left[-\delta_{0}, \delta_{0}\right]$, da equação homogênea (3.68) são da forma

$$
h_{k}(x)=\left\{\begin{array}{ll}
0, & \text { se } 0 \leqslant x \leqslant \delta_{0} \\
h_{k}(-\epsilon) e^{-k i C_{0+}(x)+\sum_{j=0}^{n-1} \lambda_{j} C_{j+}(x)}, & \text { se }-\delta_{0} \leqslant x<0
\end{array} .\right.
$$

sendo $h_{k}(-\epsilon)$ nula quando $n-j_{0}$ for ímpar. Portanto $v_{k}=u_{k}+h_{k}$ tem a forma desejada.

Proposição 3.13. Seja $\mathcal{L}_{p}$ dado por (3.2) e sejam $\lambda_{1}, \ldots, \lambda_{n-1}$ dados por (3.4). Assuma que $n<m<2 n-1$ e que $\Re\left(\lambda_{j}\right) \neq 0$ para algum $j \in\{0, \cdots, n-2\}$. Seja $j_{0}=\min _{0 \leqslant j \leqslant n-2}\{j$ : $\left.\Re\left(\lambda_{j}\right) \neq 0\right\}$. Se $\Re\left(\lambda_{j_{0}}\right) a_{0}(0)<0$ e $m-n>j_{0}$, então existe $f \in C^{\infty}\left(A_{\epsilon}\right)$, flat em $\Sigma$, tal que a equação $\mathcal{L}_{p} u=f$ não tem solução de classe $C^{\infty}$ em nenhuma vizinhança de $\Sigma$.

Demonstração. Faremos a prova por contradição.

Assuma, por absurdo, que para cada $f \in C^{\infty}\left(A_{\epsilon}\right)$ e flat em $\Sigma$ existe $u \in C^{\infty}$ tal que

$$
\mathcal{L}_{p} u=f
$$

em uma vizinhança de $\Sigma$. Logo, pela Proposição 3.4, a equação

$$
L w=\left(\sum_{j=0}^{n-1} \lambda_{j} x^{j}\right) w+g
$$

tem uma solução $w \in C^{\infty}\left(A_{\epsilon}\right)$ para cada $g$ flat em $\Sigma$.

Consequentemente, segue do Lema 3.9, que existe $\delta_{0}>0$ tal que para toda $g \in$ $C^{\infty}\left(A_{\epsilon}\right)$, flat em $\Sigma$, existe $v \in C^{\infty}\left(A_{\epsilon}\right)$ solução de

$$
L v=\left(\sum_{j=0}^{n-1} \lambda_{j} x^{j}\right) v+g
$$

em uma vizinhança de $\left[-\delta_{0}, \delta_{0}\right] \times S^{1}$. Em particular, para $g$ dada pelo Lema 3.10 existe $v \in C^{\infty}\left(A_{\epsilon}\right)$ solução de

$$
L v=\left(\sum_{j=0}^{n-1} \lambda_{j} x^{j}\right) v+g
$$


em uma vizinhança de $\left[-\delta_{0}, \delta_{0}\right] \times S^{1}$.

Usando série parcial de Fourier podemos escrever

$$
v(x, t)=\sum_{k \in \mathbb{Z}} \widehat{v}_{k}(x) e^{i k t} \quad \text { e } g(x, t)=\sum_{k \in \mathbb{Z}} \widehat{g}_{k}(x) e^{i k t}
$$

daí, concluímos que $\widehat{v}_{k}$ é uma solução de

$$
i k \widehat{v}_{k}(x)+(a+i b)(x)\left(\widehat{v}_{k}\right)^{\prime}(x)=\left(\sum_{j=0}^{n-1} \lambda_{j} x^{j}\right) \widehat{v}_{k}(x)+\widehat{g}_{k}(x)
$$

em uma vizinhança de $\left[-\delta_{0}, \delta_{0}\right] \times S^{1}$, para cada $k \in \mathbb{Z}$.

No que segue, mostraremos que existe uma escolha de $c_{k}$, na definição de $g_{k}$, que gera uma contradição com a existência de $v$ em $C^{\infty}\left(\left[-\delta_{0}, \delta_{0}\right] \times S^{1}\right)$.

Pelo Lema 3.12, para cada $k \in \mathbb{Z}$, obtemos

$$
\widehat{v}_{k}(x)=\left\{\begin{array}{ll}
e^{-i k C_{0+}(x)+\sum_{j=0}^{n-1} \lambda_{j} C_{j+}(x)} \int_{0}^{x} e^{i k C_{0+}(y)-\sum_{j=0}^{n-1} \lambda_{j} C_{j+}(y)} \frac{\widehat{g}_{k}}{a+i b}(y) \mathrm{d} y, & 0<x \leqslant \delta_{0} \\
0, & x=0 \\
H_{k} e^{-k i C_{0+}(x)+\sum_{j=0}^{n-1} \lambda_{j} C_{j+}(x)}, & -\delta_{0} \leqslant x<0
\end{array},\right.
$$

sendo $H_{k}$ uma constante que deve ser nula quando $n-j_{0}$ for ímpar.

Logo, para $0<x<\delta_{0}$, temos

$$
\widehat{v}_{k}(x)=\int_{0}^{x} e^{-i k\left(C_{0+}(x)-C_{0+}(y)\right)+\sum_{j=0}^{n-1} \lambda_{j}\left(C_{j+}(x)-C_{j+}(y)\right)} \frac{\widehat{g}_{k}}{a+i b}(y) \mathrm{d} y .
$$

Agora, observe que

$$
C_{j+}(x)-C_{j+}(y)=\int_{y}^{x} \frac{a s^{j}}{a^{2}+b^{2}}(s) \mathrm{d} s-i \int_{y}^{x} \frac{b s^{j}}{a^{2}+b^{2}}(s) \mathrm{d} s
$$

$\log \mathrm{C}$

$$
\begin{aligned}
& -i k\left(C_{0+}(x)-C_{0+}(y)\right)+\sum_{j=0}^{n-1} \lambda_{j}\left(C_{j+}(x)-C_{j+}(y)\right) \\
& =\int_{y}^{x}\left(-k+\sum_{j=0}^{n-1} \Im\left(\lambda_{j}\right) s^{j}\right) \frac{b}{a^{2}+b^{2}}(s) \mathrm{d} s+\int_{y}^{x}\left(\sum_{j=j_{0}}^{n-1} \Re\left(\lambda_{j}\right) s^{j}\right) \frac{a}{a^{2}+b^{2}}(s) \mathrm{d} s \\
& \quad+i\left(\int_{y}^{x}\left(-k+\sum_{j=0}^{n-1} \Im\left(\lambda_{j}\right) s^{j}\right) \frac{a}{a^{2}+b^{2}}(s) \mathrm{d} s-\int_{y}^{x}\left(\sum_{j=j_{0}}^{n-1} \Re\left(\lambda_{j}\right) s^{j}\right) \frac{b}{a^{2}+b^{2}}(s) \mathrm{d} s\right) .
\end{aligned}
$$


Para $y>0$ temos

$\widehat{g}_{k}(y)=e^{-\frac{1}{y}} \cdot(a+i b)(y) \cdot c_{k} \cdot e^{-i\left(\int_{y}^{x_{0}}\left(-k+\sum_{j=0}^{n-1} \Im\left(\lambda_{j}\right) s^{j}\right) \frac{a}{a^{2}+b^{2}}(s) \mathrm{d} s-\int_{y}^{x_{0}}\left(\sum_{j=j_{0}}^{n-1} \Re\left(\lambda_{j}\right) s^{j}\right) \frac{b}{a^{2}+b^{2}}(s) \mathrm{d} s\right)} ;$

obtemos

$$
\begin{aligned}
\widehat{v}_{k}\left(x_{0}\right)= & \int_{0}^{x_{0}} c_{k} e^{-k \int_{y}^{x_{0}} \frac{b}{a^{2}+b^{2}}(s) \mathrm{d} s-\frac{1}{y}+\int_{y}^{x_{0}}\left(\sum_{j=0}^{n-1} \Im\left(\lambda_{j}\right) b(s) s^{j}+\sum_{j=j_{0}}^{n-1} \Re\left(\lambda_{j}\right) a(s) s^{j}\right) \frac{1}{a^{2}+b^{2}}(s) \mathrm{d} s} \mathrm{~d} y \\
= & \int_{0}^{x_{0}}\left[c_{k} e^{-k \int_{y}^{x_{0}} \frac{b}{a^{2}+b^{2}}(s) \mathrm{d} s}\right. \\
& \left.\quad \times e^{-\frac{1}{y}+\int_{y}^{x_{0}} \frac{1}{s^{n-j_{0}}}\left(\sum_{j=0}^{n-1} \Im\left(\lambda_{j}\right) b_{0}(s) s^{j+m-n-j_{0}}+\sum_{j=j_{0}}^{n-1} \Re\left(\lambda_{j}\right) a_{0}(s) s^{j-j_{0}}\right) \frac{1}{a_{0}^{2}+x^{2(m-n)} b_{0}^{2}}(s) \mathrm{d} s}\right] \mathrm{d} y .
\end{aligned}
$$

Conforme verificado na prova do Lema 2.4 (ver página 16), temos $n-j_{0}>2$. Além disso, como $\Re\left(\lambda_{j_{0}}\right) a_{0}(0)<0$, tomando $\epsilon>0$ suficientemente pequeno, para $-\epsilon<s<\epsilon$, temos

$$
\left(\sum_{j=0}^{n-1} \Im\left(\lambda_{j}\right) b_{0}(s) s^{j+m-n-j_{0}}+\sum_{j=j_{0}}^{n-1} \Re\left(\lambda_{j}\right) a_{0}(s) s^{j-j_{0}}\right) \frac{1}{a_{0}^{2}+x^{2(m-n)} b_{0}^{2}}(s)<0
$$

concluímos que

$$
e^{-\frac{1}{y}+\int_{y}^{x_{0}} \frac{1}{s^{n-j_{0}}}\left(\sum_{j=0}^{n-1} \Im\left(\lambda_{j}\right) b_{0}(s) s^{j+m-n-j_{0}}+\sum_{j=j_{0}}^{n-1} \Re\left(\lambda_{j}\right) a_{0}(s) s^{j-j_{0}}\right) \frac{1}{a_{0}^{2}+x^{2(m-n)} b_{0}^{2}}(s) \mathrm{d} s}
$$

é limitada em $(0, \epsilon)$.

Defina

$$
M=\int_{0}^{\frac{x_{0}}{2}} e^{-\frac{1}{y}+\int_{y}^{x_{0}} \frac{1}{s^{n-j_{0}}}\left(\sum_{j=0}^{n-1} \Im\left(\lambda_{j}\right) b_{0}(s) s^{j+m-n-j_{0}}+\sum_{j=j_{0}}^{n-1} \Re\left(\lambda_{j}\right) a_{0}(s) s^{j-j_{0}}\right) \frac{1}{a_{0}^{2}+x^{2(m-n)} b_{0}^{2}}(s) \mathrm{d} s} \mathrm{~d} y>0 .
$$

Como $\frac{b}{a^{2}+b^{2}}(s)>0$ para $s>0$, temos que para $0<y_{1} \leqslant y_{2}<x_{0}$ vale:

$$
\int_{y_{1}}^{x_{0}} \frac{b}{a^{2}+b^{2}}(s) \mathrm{d} s \geqslant \int_{y_{2}}^{x_{0}} \frac{b}{a^{2}+b^{2}}(s) \mathrm{d} s
$$

Logo, se $k<0$, temos

$$
\begin{aligned}
\widehat{v}_{k}\left(x_{0}\right) & \geqslant M \int_{0}^{\frac{x_{0}}{2}} c_{k} e^{-k \int_{y}^{x_{0}} \frac{b}{a^{2}+b^{2}}(s) \mathrm{d} s} \mathrm{~d} y \\
& \geqslant M \int_{0}^{\frac{x_{0}}{2}} c_{k} e^{-k \int_{\frac{x_{0}}{2}}^{x_{0}} \frac{b}{a^{2}+b^{2}}(s) \mathrm{d} s} \mathrm{~d} y
\end{aligned}
$$




$$
=c_{k} e^{-k M^{\prime}} M
$$

sendo $M^{\prime}=\int_{\frac{x_{0}}{2}}^{x_{0}} \frac{b}{a^{2}+b^{2}}(s) \mathrm{d} s>0$.

Se tomarmos, na definição de $g_{k}, c_{k}=e^{k M^{\prime}}$ para cada $k<0$, teremos $\widehat{v}_{k}\left(x_{0}\right) \geqslant M>0$ e, consequentemente, $\left(\widehat{v}_{k}\right)$ não pode ser a sequência de Fourier de nenhuma função $C^{\infty}$ periódica. Assim concluímos que não existe $v \in C^{\infty}\left(\left[-\delta_{0}, \delta_{0}\right] \times S^{1}\right)$ solução de $L v=\left(\sum_{j=0}^{n-1} \lambda_{j} x^{j}\right) v+g$ em uma vizinhança de $\left[-\delta_{0}, \delta_{0}\right] \times S^{1}$, o que é uma contradição.

Lema 3.14. Seja $\delta_{0}>0$ dado pelo Lema 3.9 e seja $x_{0} \in\left(-\delta_{0}, 0\right)$. Para cada $k \in \mathbb{Z}$, seja

$$
g_{k}:(-\epsilon, \epsilon) \rightarrow \mathbb{C}
$$

definida por

$$
g_{k}(x)=\varphi_{1}(x) \cdot(a+i b)(x) \cdot c_{k} \cdot \varphi_{2}(x)
$$

sendo $\left(c_{k}\right)$ uma sequência de números reais positivos rapidamente decrescente, $\varphi_{1}:(-\epsilon, \epsilon) \rightarrow \mathbb{R}$ dada por

$$
\varphi_{1}(x)= \begin{cases}0, & x \geqslant 0 \\ -e^{\frac{1}{x}}, & x<0\end{cases}
$$

$e \varphi_{2}:(-\epsilon, \epsilon) \rightarrow \mathbb{C}$ dada por

$$
\varphi_{2}(x)= \begin{cases}0, & x \geqslant 0 \\ e^{i\left(\int_{x_{0}}^{x}\left(-k+\sum_{j=0}^{n-1} \Im\left(\lambda_{j}\right)_{j} s^{j}\right) \frac{a}{a^{2}+b^{2}}(s) \mathrm{d} s-\int_{x_{0}}^{x}\left(\sum_{j=j_{0}}^{n-1} \Re\left(\lambda_{j}\right) s^{j}\right) \frac{b}{a^{2}+b^{2}}(s) \mathrm{d} s\right)}, & x<0 .\end{cases}
$$

A sequência $\left(g_{k}\right)$ define uma função $g \in C^{\infty}\left(A_{\epsilon}\right)$ e flat em $\Sigma$.

A demonstração do Lema 3.14 utiliza os mesmos argumentos da demonstração do Lema 3.10; vamos omiti-la.

Lema 3.15. Assuma que $n<m<2 n-1$ e que $\Re\left(\lambda_{j}\right) \neq 0$ para algum $j \in\{0, \cdots, n-2\}$. Seja $j_{0}=\min _{0 \leqslant j \leqslant n-2}\left\{j: \Re\left(\lambda_{j}\right) \neq 0\right\}$, e seja $\delta_{0}>0$ dado pelo Lema 3.9. Para cada $k \in \mathbb{Z}$, seja $g_{k}$ dado pelo Lema 3.14. Seja $j_{0}=\min _{0 \leqslant j \leqslant n-2}\left\{j: \Re\left(\lambda_{j}\right) \neq 0\right\}$. Suponha que $\Re\left(\lambda_{j}\right) \neq 0$ para algum $j \in\{0, \cdots, n-2\}$. Se $\Re\left(\lambda_{j_{0}}\right) a_{0}(0)>0, n-j_{0}$ é par e $m-n>j_{0}$, então as soluções de classe $C^{\infty}$ de

$$
i k v_{k}(x)+(a+i b)(x)\left(v_{k}\right)^{\prime}(x)=\left(\sum_{j=0}^{n-1} \lambda_{j} x^{j}\right) v_{k}+g_{k}(x)
$$


em uma vizinhança de $\left[-\delta_{0}, \delta_{0}\right]$, são da forma

$$
v_{k}(x)=\left\{\begin{array}{ll}
H_{k} e^{-k i C_{0+}(x)+\sum_{j=0}^{n-1} \lambda_{j} C_{j+}(x)}, & 0<x \leqslant \delta_{0} \\
0, & x=0 \\
-e^{-i k C_{0-}(x)+\sum_{j=0}^{n-1} \lambda_{j} C_{j-}(x)} \int_{x}^{0} e^{i k C_{0-}(y)-\sum_{j=0}^{n-1} \lambda_{j} C_{j-}(y)} \frac{\widehat{g}_{k}}{a+i b}(y) \mathrm{d} y & -\delta_{0} \leqslant x<0
\end{array},\right.
$$

sendo $H_{k}$ uma constante.

Demonstração. A prova é similar àquela do Lema 3.12.

Seja $v_{k} \in C^{\infty}((-\epsilon, \epsilon))$ solução de $(3.71)$ em uma vizinhança de $\left[-\delta_{0}, \delta_{0}\right]$. Então, $v_{k}$ tem a forma $v_{k}=u_{k}+h_{k}(x)$, sendo $h_{k}$ uma solução arbitrária da equação homogênea

$$
i k h_{k}(x)+(a+i b)(x)\left(h_{k}\right)^{\prime}(x)=\left(\sum_{j=0}^{n-1} \lambda_{j} x^{j}\right) h_{k}(x)
$$

e $u_{k}$ uma solução particular da equação não homogênea (3.71).

Afirmamos que $e^{i k C_{0+}(y)-\sum_{j=0}^{n-1} \lambda_{j} C_{j+}(y)}$ é limitada em $(-\epsilon, 0)$. De fato, pelo Lema 3.11, existem $\epsilon_{k}>0$ e $\varphi_{k} \in C^{\infty}((-\epsilon, \epsilon))$ tais que, para $-\epsilon_{k}<y<0$, obtemos

$$
\begin{aligned}
\left|e^{i k C_{0}-(y)-\sum_{j=0}^{n-1} \lambda_{j} C_{j-}(y)}\right| & =e^{\frac{-\Re\left(\lambda_{j_{0}}\right)}{a_{0}(0)} \int_{-\epsilon}^{-\epsilon_{k}} \frac{1}{s^{n-j_{0}}} \varphi_{k}(s) \mathrm{d} s} e^{\frac{-\Re\left(\lambda_{j_{0}}\right)}{a_{0}(0)} \int_{-\epsilon_{k}}^{y} \frac{1}{s^{n-j_{0}}} \varphi_{k}(s) \mathrm{d} s} \\
& \leqslant M_{k} e^{\frac{-\Re\left(\lambda_{j_{0}}\right)}{a_{0}(0)} \frac{1}{2} \int_{-\epsilon_{k}}^{y} \frac{1}{s^{n-j_{0}}} \mathrm{~d} s}=M_{k} e^{\frac{\Re\left(\lambda_{j_{0}}\right)}{2 a_{0}(0)}\left(\left.\frac{1}{\left(n-j_{0}-1\right)} \frac{1}{s^{n-j_{0}-1}}\right|_{-\epsilon_{k}} ^{y}\right.} \\
& =M_{k} e^{\frac{\Re\left(\lambda_{j_{0}}\right)}{2 a_{0}(0)\left(n-j_{0}-1\right)}}\left(\frac{1}{y^{n-j_{0}-1}-\frac{1}{\left(-\epsilon_{k}\right)^{n-j_{0}-1}}}\right)
\end{aligned}
$$

sendo $M_{k}=e^{\frac{-\Re\left(\lambda_{j_{0}}\right)}{a_{0}(0)} \int_{-\epsilon}^{-\epsilon_{k}} \frac{1}{s^{n-j_{0}}} \varphi_{k}(s) \mathrm{d} s}$.

Assim, para $-\epsilon_{k}<y<0$, temos

$$
\left|e^{i k C_{0-}(y)-\sum_{j=0}^{n-1} \lambda_{j} C_{j-}(y)}\right| \leqslant M_{k}^{\prime} e^{\frac{\Re\left(\lambda_{j_{0}}\right)}{2 a_{0}(0)\left(n-j_{0}-1\right)} \frac{1}{y^{n-j_{0}-1}}},
$$

sendo $M_{k}^{\prime}=M_{k} e^{-\frac{\Re\left(\lambda_{j_{0}}\right)}{2 a_{0}(0)\left(n-j_{0}-1\right)} \frac{1}{\left(-\epsilon_{k}\right)^{n-j_{0}-1}}} ;$ note que $n-j_{0}>2$.

Como $\frac{\Re\left(\lambda_{j_{0}}\right)}{a_{0}(0)}>0$ e $n-j_{0}-1$ é ímpar e positivo, concluímos que $e^{i k C_{0+}(y)-\sum_{j=0}^{n-1} \lambda_{j} C_{j+}(y)}$ é limitada em $(0, \epsilon)$, para cada $k \in \mathbb{Z}$; consequentemente $e^{i k C_{0+}(y)-\sum_{j=0}^{n-1} \lambda_{j} C_{j+}(y)}$ é integrável em $\left[-\delta_{0}, 0\right]$.

Logo, para $x<0$, encontramos

$$
u_{k}(x)=-e^{-i k C_{0+}(x)+\sum_{j=0}^{n-1} \lambda_{j} C_{j+}(x)} \int_{x}^{0} e^{i k C_{0-}(y)-\sum_{j=0}^{n-1} \lambda_{j} C_{j}(y)} \frac{g_{k}}{a+i b}(y) \mathrm{d} y .
$$


Afirmamos que $\left|u_{k}(x)\right|=O\left(|x|^{j}\right), \forall j \in \mathbb{Z}_{+}$.

De fato. Para $-\epsilon_{k}<x<y<0$, de (3.65), temos

$$
\left|\frac{e^{i k C_{0-}(y)-\sum_{j=0}^{n-1} \lambda_{j} C_{j-}(y)}}{e^{i k C_{0-}(x)-\sum_{j=0}^{n-1} \lambda_{j} C_{j-}(x)}}\right|=e^{\frac{-\Re\left(\lambda_{j_{0}}\right)}{a_{0}(0)} \int_{x}^{y} \frac{1}{s^{n-j_{0}}} \varphi_{k}(s) \mathrm{d} s} \leqslant e^{\frac{-\Re\left(\lambda_{j_{0}}\right)}{2 a_{0}(0)} \int_{x}^{y} \frac{1}{s^{n-j_{0}}} \mathrm{~d} s} \leqslant 1 ;
$$

consequentemente, para $-\epsilon_{k}<x<0$, temos

$$
\begin{aligned}
\left|u_{k}(x)\right| \leqslant \int_{x}^{0}\left|\frac{e^{i k C_{0}-(y)-\sum_{j=0}^{n-1} \lambda_{j} C_{j-}(y)}}{e^{i k C_{0}-(x)-\sum_{j=0}^{n-1} \lambda_{j} C_{j-}(x)}} \frac{\widehat{g}_{k}}{a+i b}\right| & (y) \mathrm{d} y \\
& \leqslant \int_{x}^{0}\left|\frac{\widehat{g}_{k}}{a+i b}\right|(y) \mathrm{d} y=O\left(|x|^{j}\right), \forall j \in \mathbb{Z}_{+} .
\end{aligned}
$$

Note que estimativas análogas podem ser provadas para as derivadas $u_{k}^{(\nu)}, \nu \in \mathbb{Z}_{+}$, na região $x>0$.

Concluímos, então, que $u_{k}$ definida por

$$
u_{k}(x) \doteq \begin{cases}0, & x \geqslant 0 \\ -e^{-i k C_{0+}(x)+\sum_{j=0}^{n-1} \lambda_{j} C_{j+}(x)} \int_{x}^{0} e^{i k C_{0-}(y)-\sum_{j=0}^{n-1} \lambda_{j} C_{j}(y)} \frac{g_{k}}{a+i b}(y) \mathrm{d} y, & x<0\end{cases}
$$

é uma solução particular, de classe $C^{\infty}$, da equação não homogênea (3.71).

Agora, vamos analisar a solução $h_{k}$ da equação homogênea (3.72). Simples cálculos mostram que a solução da equação homogênea é dada por

$$
h_{k}(x)=h_{k}(\epsilon) e^{-i k C_{0+}(x)+\sum_{j=0}^{n-1} \lambda_{j} C_{j+}(x)} \quad \text { se } \quad 0<x<\epsilon,
$$

$\mathrm{e}$

$$
h_{k}(x)=h_{k}(-\epsilon) e^{i k C_{0-}(x)+\sum_{j=0}^{n-1} \lambda_{j} C_{j-}(x)}, \quad \text { se } \quad-\epsilon<x<0 .
$$

Afirmamos que $h_{k}(x) \notin C^{\infty}$ se $h_{k}(-\epsilon) \neq 0$.

De fato, para $-\epsilon_{-k}<x<0$, por (3.73) temos

$$
\left|h_{k}(x)\right|=\frac{\left|h_{k}(-\epsilon)\right|}{\left|e^{i k C_{0-}(x)-\sum_{j=N}^{n-1} \lambda_{j} C_{j-}(x)}\right|} \geqslant \frac{\left|h_{k}(-\epsilon)\right|}{M_{-k}^{\prime}} e^{\frac{-\Re\left(\lambda_{j_{0}}\right)}{2 a_{0}(0)\left(n-j_{0}-1\right)}} \frac{1}{x^{n-j_{0}-1}} .
$$

Logo, $h_{k}$ não é contínua na origem se $h_{k}(-\epsilon) \neq 0$, pois $\frac{\Re\left(\lambda_{j_{0}}\right)}{a_{0}(0)}>0$ e $n-j_{0}-1$ é ímpar positivo.

Portanto, da afirmação acima e da prova da Proposição 2.4, concluímos que $h_{k}(x) \in$ $C^{\infty}$ se, e somente se, $h_{k}(-\epsilon)=0$. 
Assim, concluímos que as soluções de classe $C^{\infty}$, em uma vizinhança de $\left[-\delta_{0}, \delta_{0}\right]$, da equação homogênea (3.72) são da forma

$$
h_{k}(x)=\left\{\begin{array}{ll}
H_{k} e^{-i k C_{0+}(x)+\sum_{j=0}^{n-1} \lambda_{j} C_{j+}(x)}, & \text { se } 0<x \leqslant \delta_{0} \\
0 & \text { se }-\delta_{0} \leqslant x \leqslant 0
\end{array} ;\right.
$$

portanto $v_{k}=u_{k}+h_{k}$ tem a forma desejada.

Proposição 3.16. Seja $\mathcal{L}_{p}$ dado por (3.2) e sejam $\lambda_{1}, \ldots, \lambda_{n-1}$ dados por (3.4). Assuma que $n<m<2 n-1$ e que $\Re\left(\lambda_{j}\right) \neq 0$ para algum $j \in\{0, \cdots, n-2\}$. Seja $j_{0}=\min _{0 \leqslant j \leqslant n-2}\left\{j: \Re\left(\lambda_{j}\right) \neq 0\right\} . S e \Re\left(\lambda_{j_{0}}\right) a_{0}(0)>0, n-j_{0}$ é par, e $m-n>j_{0}$, então existe $f \in C^{\infty}\left(A_{\epsilon}\right)$, flat em $\Sigma$, tal que a equação $\mathcal{L}_{p} u=f$ não tem solução de classe $C^{\infty}$ em nenhuma vizinhança de $\Sigma$.

Demonstração. Utilizaremos um procedimento similar ao da prova da Proposição 3.13. Entretanto, agora vamos nos concentrar na região $x<0$ e utilizar $g$ definida no Lema 3.14.

Assuma, por absurdo, que para cada $f \in C^{\infty}\left(A_{\epsilon}\right)$ e flat em $\Sigma$ existe $u \in C^{\infty}$ tal que

$$
\mathcal{L}_{p} u=f
$$

em uma vizinhança de $\Sigma$. Logo, pela Proposição 3.4, a equação

$$
L w=\left(\sum_{j=0}^{n-1} \lambda_{j} x^{j}\right) w+g
$$

tem uma solução $w \in C^{\infty}\left(A_{\epsilon}\right)$ para cada $g \in C^{\infty}\left(A_{\epsilon}\right)$ flat em $\Sigma$.

Consequentemente, segue do Lema 3.9, que existe $\delta_{0}>0$ tal que para toda $g \in$ $C^{\infty}\left(A_{\epsilon}\right)$, flat em $\Sigma$, existe $v \in C^{\infty}\left(A_{\epsilon}\right)$ solução de

$$
L v=\left(\sum_{j=0}^{n-1} \lambda_{j} x^{j}\right) v+g
$$

em uma vizinhança de $\left[-\delta_{0}, \delta_{0}\right] \times S^{1}$. Em particular, para $g$ dada pelo Lema 3.14 existe $v \in C^{\infty}\left(A_{\epsilon}\right)$ solução de

$$
L v=\left(\sum_{j=0}^{n-1} \lambda_{j} x^{j}\right) v+g
$$

em uma vizinhança de $\left[-\delta_{0}, \delta_{0}\right] \times S^{1}$. 
Usando série parcial de Fourier, podemos escrever

$$
v(x, t)=\sum_{k \in \mathbb{Z}} \widehat{v}_{k}(x) e^{i k t} \quad \text { e } g(x, t)=\sum_{k \in \mathbb{Z}} \widehat{g}_{k}(x) e^{i k t}
$$

logo, temos que $\widehat{v}_{k}$ é uma solução de

$$
i k \widehat{v}_{k}(x)+(a+i b)(x)\left(\widehat{v}_{k}\right)^{\prime}(x)=\left(\sum_{j=0}^{n-1} \lambda_{j} x^{j}\right) \widehat{v}_{k}(x)+\widehat{g}_{k}(x)
$$

em uma vizinhança de $\left[-\delta_{0}, \delta_{0}\right]$, para cada $k \in \mathbb{Z}$.

No que segue, mostraremos que existe uma escolha de $c_{k}$, na definição de $g_{k}$, que gera uma contradição com a existência de $v$ em $C^{\infty}\left(\left[-\delta_{0}, \delta_{0}\right] \times S^{1}\right)$.

Pelo Lema 3.15, para cada $k \in \mathbb{Z}$, obtemos

$$
\widehat{v}_{k}(x)= \begin{cases}H_{k} e^{-k i C_{0+}(x)+\sum_{j=0}^{n-1} \lambda_{j} C_{j+}(x)}, & 0<x \leqslant \delta_{0} \\ 0, & x=0 \\ -e^{-i k C_{0-}(x)+\sum_{j=0}^{n-1} \lambda_{j} C_{j-}(x)} \int_{x}^{0} e^{i k C_{0-}(y)-\sum_{j=0}^{n-1} \lambda_{j} C_{j-}(y)} \frac{\widehat{g}_{k}}{a+i b}(y) \mathrm{d} y & -\delta_{0} \leqslant x<0\end{cases}
$$

sendo $H_{k}$ uma constante.

Logo, para $-\delta_{0} \leqslant x<0$, temos

$$
\widehat{v}_{k}(x)=-\int_{x}^{0} e^{-i k\left(C_{0-}(x)-C_{0-}(y)\right)+\sum_{j=0}^{n-1} \lambda_{j}\left(C_{j-}(x)-C_{j-}(y)\right)} \frac{\widehat{g}_{k}}{a+i b}(y) \mathrm{d} y .
$$

Agora, observe que

$$
\begin{aligned}
& -i k\left(C_{0-}(x)-C_{0-}(y)\right)+\sum_{j=0}^{n-1} \lambda_{j}\left(C_{j-}(x)-C_{j-}(y)\right) \\
& =-\int_{x}^{y}\left(-k+\sum_{j=0}^{n-1} \Im\left(\lambda_{j}\right) s^{j}\right) \frac{b}{a^{2}+b^{2}}(s) \mathrm{d} s-\int_{x}^{y}\left(\sum_{j=j_{0}}^{n-1} \Re\left(\lambda_{j}\right) s^{j}\right) \frac{a}{a^{2}+b^{2}}(s) \mathrm{d} s \\
& \quad-i\left(\int_{x}^{y}\left(-k+\sum_{j=0}^{n-1} \Im\left(\lambda_{j}\right) s^{j}\right) \frac{a}{a^{2}+b^{2}}(s) \mathrm{d} s-\int_{x}^{y}\left(\sum_{j=j_{0}}^{n-1} \Re\left(\lambda_{j}\right) s^{j}\right) \frac{b}{a^{2}+b^{2}}(s) \mathrm{d} s\right) .
\end{aligned}
$$

Para $y<0$ temos que

$$
\widehat{g}_{k}(y)=-e^{\frac{1}{y}} \cdot(a+i b)(y) \cdot c_{k} \cdot e^{i\left(\int_{x_{0}}^{y}\left(-k+\sum_{j=0}^{n-1} \Im\left(\lambda_{j}\right) s^{j}\right) \frac{a}{a^{2}+b^{2}}(s) \mathrm{d} s-\int_{x_{0}}^{y}\left(\sum_{j=j_{0}}^{n-1} \Re\left(\lambda_{j}\right) s^{j}\right) \frac{b}{a^{2}+b^{2}}(s) \mathrm{d} s\right)} ;
$$


obtemos

$$
\begin{aligned}
\widehat{v}_{k}\left(x_{0}\right) & =\int_{x_{0}}^{0} c_{k} e^{k \int_{x_{0}}^{y} \frac{b}{a^{2}+b^{2}}(s) \mathrm{d} s+\frac{1}{y}-\int_{x_{0}}^{y}\left(\sum_{j=0}^{n-1} \Im\left(\lambda_{j}\right) b(s) s^{j}+\sum_{j=j_{0}}^{n-1} \Re\left(\lambda_{j}\right) a(s) s^{j}\right) \frac{1}{a^{2}+b^{2}}(s) \mathrm{d} s} \mathrm{~d} y \\
& =\int_{x_{0}}^{0} c_{k} e^{\int_{x_{0}}^{y} k \frac{b}{a^{2}+b^{2}}(s) \mathrm{d} s} \\
& \times e^{\frac{1}{y}-\int_{x_{0}}^{y} \frac{1}{s^{n-j_{0}}}\left(\sum_{j=0}^{n-1} \Im\left(\lambda_{j}\right) b_{0}(s) s^{j+m-n-j_{0}}+\sum_{j=j_{0}}^{n-1} \Re\left(\lambda_{j}\right) a_{0}(s) s^{j-j_{0}}\right) \frac{1}{a_{0}^{2}+x^{2(m-n)} b_{0}^{2}}(s) \mathrm{d} s} \mathrm{~d} y .
\end{aligned}
$$

Temos $n-j_{0}>2$, além disso, como $\Re\left(\lambda_{j_{0}}\right) a_{0}(0)>0$, tomando $\epsilon>0$ suficientemente pequeno, para $-\epsilon<s<\epsilon$, temos

$$
\left(\sum_{j=0}^{n-1} \Im\left(\lambda_{j}\right) b_{0}(s) s^{j+m-n-j_{0}}+\sum_{j=j_{0}}^{n-1} \Re\left(\lambda_{j}\right) a_{0}(s) s^{j-j_{0}}\right) \frac{1}{a_{0}^{2}+x^{2(m-n)} b_{0}^{2}}(s)>0
$$

então, temos

$$
e^{\frac{1}{y}-\int_{x_{0}}^{y} \frac{1}{s^{n-j_{0}}}\left(\sum_{j=0}^{n-1} \Im\left(\lambda_{j}\right) b_{0}(s) s^{j+m-n-j_{0}}+\sum_{j=j_{0}}^{n-1} \Re\left(\lambda_{j}\right) a_{0}(s) s^{j-j_{0}}\right) \frac{1}{a_{0}^{2}+x^{2(m-n)} b_{0}^{2}}(s) \mathrm{d} s}
$$

é limitada em $(-\epsilon, 0)$, já que $n-j_{0}$ é par por hipótese.

Defina

$$
M=\int_{x_{0}}^{0} e^{\frac{1}{y}-\int_{x_{0}}^{y} \frac{1}{s^{n-j_{0}}}\left(\sum_{j=0}^{n-1} \Im\left(\lambda_{j}\right) b_{0}(s) s^{j+m-n-j_{0}}+\sum_{j=j_{0}}^{n-1} \Re\left(\lambda_{j}\right) a_{0}(s) s^{j-j_{0}}\right) \frac{1}{a_{0}^{2}+x^{2(m-n)} b_{0}^{2}}(s) \mathrm{d} s} \mathrm{~d} y .
$$

Logo,

$$
\widehat{v}_{k}\left(x_{0}\right)=M \int_{x_{0}}^{0} c_{k} e^{\int_{x_{0}}^{y} k \frac{b}{a^{2}+b^{2}}(s) \mathrm{d} s}
$$

Note que $\frac{b_{0}}{a^{2}+b^{2}}>0$ por hipótese. Logo, tomando $\epsilon>0$ suficientemente pequeno, existem $\alpha, \beta>0$ tais que, para $-\epsilon<s<\epsilon$ vale:

$$
\alpha \leqslant \frac{b_{0}}{a_{0}^{2}+s^{2 m-2 n} b_{0}^{2}} \leqslant \beta
$$

Suponha que $m$ é ímpar; consequentemente $s^{2 n-m}<0$ se $-\epsilon<s<0$. Então, para $k>0$, temos que para $-\delta_{0}<x_{0}<y<0$ vale:

$$
\begin{aligned}
k \int_{x_{0}}^{y} \frac{b}{a^{2}+b^{2}}(s) \mathrm{d} s & =k \int_{x_{0}}^{y} \frac{1}{s^{2 n-m}} \frac{b_{0}}{a_{0}^{2}+s^{2 m-2 n} b_{0}^{2}}(s) \mathrm{d} s \\
& \geqslant k \beta \int_{x_{0}}^{y} \frac{1}{s^{2 n-m}}(s) \mathrm{d} s=\frac{k \beta}{(2 n-m-1)}\left(\frac{1}{x_{0}^{2 n-m-1}}-\frac{1}{y^{2 n-m-1}}\right) ;
\end{aligned}
$$


consequentemente, de (3.74), temos

$$
\begin{aligned}
\widehat{v}_{k}\left(x_{0}\right) & =M \int_{x_{0}}^{0} c_{k} e^{k \int_{x_{0}}^{y} \frac{b}{a^{2}+b^{2}}(s) \mathrm{d} s} \mathrm{~d} y \geqslant M \int_{x_{0}}^{0} c_{k} e^{\frac{k \beta}{(2 n-m-1)}\left(\frac{1}{\left.x_{0}^{2 n-m-1}-\frac{1}{y^{2 n-m-1}}\right)} \mathrm{d} y\right.} \\
& =c_{k} e^{k M_{1}} M_{2},
\end{aligned}
$$

sendo $M_{1}=\frac{k \beta}{(2 n-m-1) x_{0}^{2 n-m-1}}>0$ e $M_{2}=\int_{x_{0}}^{0} e^{\frac{-k \beta}{(2 n-m-1)} \frac{1}{y^{2 n-m-1}}} \mathrm{~d} y>0$.

Logo, se $m$ é ímpar, tomando (na definição de $g_{k}$ ), $c_{k}=e^{-k M}$ para cada $k>0$, teremos $\widehat{v}_{k}\left(x_{0}\right) \geqslant M_{2}>0$; consequentemente $\left(\widehat{v}_{k}\right)$ não pode ser a sequência de Fourier de nenhuma função $C^{\infty}$ periódica.

Agora, suponha que $m$ é par; consequentemente $\frac{b}{a^{2}+b^{2}}=s^{m} \frac{b_{0}}{a^{2}+b^{2}}>0$. Logo, para $x_{0}<y_{1} \leqslant y_{2}<0$, temos

$$
\int_{x_{0}}^{y_{1}} \frac{b}{a^{2}+b^{2}}(s) \mathrm{d} s \leqslant \int_{x_{0}}^{y_{2}} \frac{b}{a^{2}+b^{2}}(s) \mathrm{d} s
$$

Portanto, para $k>0$ e $m$ par, segue de (3.74) que vale:

$$
\begin{aligned}
\widehat{v}_{k}\left(x_{0}\right) & \geqslant M \int_{\frac{x_{0}}{2}}^{0} c_{k} e^{k \int_{x_{0}}^{y} \frac{b}{a^{2}+b^{2}}(s) \mathrm{d} s} \mathrm{~d} y \\
& \geqslant M \int_{\frac{x_{0}}{2}}^{0} c_{k} e^{k \int_{x_{0}}^{\frac{x_{0}}{2}} \frac{b}{a^{2}+b^{2}}(s) \mathrm{d} s} \mathrm{~d} y \\
& =c_{k} e^{k M_{3}} M,
\end{aligned}
$$

sendo $M_{3}=\int_{x_{0}}^{\frac{x_{0}}{2}} \frac{b}{a^{2}+b^{2}}(s) \mathrm{d} s>0$.

Logo, se $m$ é par, tomando (na definição de $g_{k}$ ), $c_{k}=e^{-k M_{3}}$ para cada $k>0$, teremos $\widehat{v}_{k}\left(x_{0}\right) \geqslant M>0$; consequentemente $\left(\widehat{v}_{k}\right)$ não pode ser a sequência de Fourier de nenhuma função $C^{\infty}$ periódica.

Portanto, não existe $v \in C^{\infty}\left(\left[-\delta_{0}, \delta_{0}\right] \times S^{1}\right)$ solução de $L v=\left(\sum_{j=0}^{n-1} \lambda_{j} x^{j}\right) v+g$ em uma vizinhança de $\left[-\delta_{0}, \delta_{0}\right] \times S^{1}$, o que é uma contradição.

Agora, mostraremos que o subespaço $\mathcal{F}$ de $C^{\infty}\left(A_{\epsilon}\right)$, das funções $f$ que satisfazem as condições de compatibilidade da equação $\mathcal{L}_{p} u=f$, tem codimensão finita.

Lema 3.17. Sejam $\lambda_{1}, \ldots, \lambda_{r-1}$ dados por (3.4), e seja $\mathcal{F} \subset C^{\infty}\left(A_{\epsilon}\right)$ definido por:

(1) $\mathcal{F}=C^{\infty}\left(A_{\epsilon}\right)$ se $\lambda_{0} \notin i \mathbb{Z}$. 
(2) $\mathcal{F}$ é o subespaço de $C^{\infty}\left(A_{\epsilon}\right)$ de todas funções $f$ satisfazendo (3.13) se $\lambda_{0} \in i \mathbb{Z} e$ $1 \leqslant N<r-1$, ou ainda se $\lambda_{0} \in i \mathbb{Z}, N=r-1$ e $\lambda_{r-1}-\ell \alpha_{0} \neq 0 \forall \ell \in \mathbb{Z}_{+}$, sendo $N$ o menor inteiro, satisfazendo $1 \leqslant N \leqslant r-1$, tal que $\lambda_{N} \neq 0$.

(3) $\mathcal{F}$ é o subespaço de $C^{\infty}\left(A_{\epsilon}\right)$ de todas funções $f$ satisfazendo (3.9) se as condições (3.7), (3.8) são satisfeitas.

(4) $\mathcal{F}$ é o subespaço de $C^{\infty}\left(A_{\epsilon}\right)$ de todas funções $f$ satisfazendo, além da condição (3.13), a condição adicional (3.14) descrita na demonstração da Proposição 3.3 se $\lambda_{0} \in i \mathbb{Z}, N=r-1$ e $\lambda_{r-1}-\ell_{0} \alpha_{0}=0$, para algum $\ell_{0} \in \mathbb{Z}_{+}$, sendo $N$ o menor inteiro, satisfazendo $1 \leqslant N \leqslant r-1$, tal que $\lambda_{N} \neq 0$.

Então, $\mathcal{F}$ é um subespaço de codimensão finita de $C^{\infty}\left(A_{\epsilon}\right)$.

Demonstração. Se $\mathcal{F}=C^{\infty}\left(A_{\epsilon}\right)$ então $\mathcal{F}$ tem codimensão igual a zero; portanto finita.

Suponha que $\mathcal{F}$ definido em $(2)$.

Defina

$$
Q_{N-1}(x, t) \doteq \sum_{j=0}^{N-1}\left(\int_{0}^{t} \frac{\partial^{j} p}{\partial x^{j}}(0, \sigma) d \sigma\right) x^{j}
$$

Então, $\mathcal{F}=S^{\circ}$, sendo

$$
S=\operatorname{span}\left\langle e^{-Q_{N-1}}\left(1 \otimes \delta^{(j)}\right), j=0, \ldots, N-1\right\rangle
$$

e

$$
S^{\circ} \doteq\left\{f \in C^{\infty}\left(A_{\epsilon}\right) ;\langle u, f\rangle=0, \forall u \in S\right\}
$$

Agora, vamos mostrar que $S^{\circ}$ tem codimensão finita. Para cada $j=0, \ldots, N-1$ defina

$$
S_{j} \doteq \operatorname{span}\left\langle e^{-Q_{N-1}}\left(1 \otimes \delta^{(k)}\right), k=0, \ldots, \widehat{j}, \ldots, N-1\right\rangle
$$

Como $S_{j} \subset \mathcal{E}^{\prime}\left(A_{\epsilon}\right)$ é fechado, $e^{-Q_{N-1}}\left(1 \otimes \delta^{(j)}\right) \notin \overline{S_{j}}$. Logo, pelo Teorema de HahnBanach (ver, por exemplo, Teorema 3.5 de [11]), existe $\Lambda$ no dual de $\mathcal{E}^{\prime}\left(A_{\epsilon}\right)$ para o qual $\Lambda\left[e^{-Q_{N-1}}\left(1 \otimes \delta^{(j)}\right)\right]=1$ e, além disso, se $\ell \in\{0, \ldots, N-1\}, \ell \neq j$ então $\Lambda\left[e^{-Q_{N-1}}\left(1 \otimes \delta^{(\ell)}\right)\right]=0$.

Note que o dual de $C^{\infty}\left(A_{\epsilon}\right)$ é $\mathcal{E}^{\prime}\left(A_{\epsilon}\right)$. Como $C^{\infty}\left(A_{\epsilon}\right)$ é reflexivo, existe um isomorfismo isométrico (ver, por exemplo, página 90 de [11])

$$
\phi: C^{\infty}\left(A_{\epsilon}\right) \rightarrow\left[\mathcal{E}^{\prime}\left(A_{\epsilon}\right)\right]^{\prime}
$$




$$
f \mapsto \phi(f)=\phi_{f}
$$

tal que $\phi\left[C^{\infty}\left(A_{\epsilon}\right)\right]=\left[\mathcal{E}^{\prime}\left(A_{\epsilon}\right)\right]^{\prime}$, sendo

$$
\begin{aligned}
\phi_{f}:\left[\mathcal{E}^{\prime}\left(A_{\epsilon}\right)\right]^{\prime} & \rightarrow \mathbb{C} \\
\mu & \mapsto \phi_{f}(\mu)=\langle\mu, f\rangle .
\end{aligned}
$$

Logo, concluímos que existe $\varphi_{j} \in C^{\infty}\left(A_{\epsilon}\right)$ tal que, para cada $\ell=0, \ldots, N-1$, temos

$$
\left\langle e^{-Q_{N-1}}\left(1 \otimes \delta^{(\ell)}\right), \varphi_{j}\right\rangle= \begin{cases}0, & \text { se } \ell \neq j \\ 1, & \text { se } \ell=j\end{cases}
$$

Afirmamos que $C^{\infty}\left(A_{\epsilon}\right)=S^{\circ} \oplus E$, sendo $E=\operatorname{span}\left\langle\varphi_{0}, \ldots, \varphi_{N-1}\right\rangle$.

De fato, se $g \in S^{\circ} \cap E$ então $\left\langle e^{-Q_{N-1}}\left(1 \otimes \delta^{(j)}\right), g\right\rangle=0, j=0 \ldots, N-1$, e existem $\alpha_{0}, \ldots, \alpha_{N-1}$, tais que $g=\alpha_{0} \varphi_{0}+\cdots+\alpha_{N-1} \varphi_{N-1}$.

Logo, $0=\left\langle e^{-Q_{N-1}}\left(1 \otimes \delta^{(j)}\right), g\right\rangle=\alpha_{j}, j=0 \ldots, N-1$, ou seja, $S^{\circ} \cap E=\{0\}$.

Falta mostrar que $C^{\infty}\left(A_{\epsilon}\right) \subset S^{\circ} \oplus E$. Dado $g \in C^{\infty}\left(A_{\epsilon}\right)$, defina $\beta_{j} \doteq\left\langle e^{-Q_{N-1}}(1 \otimes\right.$ $\left.\left.\delta^{(j)}\right), g\right\rangle, j=0 \ldots, N-1$ e $\psi \doteq g-\sum_{j=0}^{N-1} \beta_{j} \varphi_{j}$. Então, para cada $j=0 \ldots, N-1$, temos

$$
\left\langle e^{-Q_{N-1}}\left(1 \otimes \delta^{(j)}\right), \psi\right\rangle=\left\langle e^{-Q_{N-1}}\left(1 \otimes \delta^{(j)}\right), g\right\rangle-\beta_{j}=\beta_{j}-\beta_{j}=0
$$

Portanto, cada $g \in C^{\infty}\left(A_{\epsilon}\right)$ se escreve como $g=\psi+\varphi$, $\operatorname{com} \psi \in S^{\circ}$ e $\varphi \doteq$ $\sum_{j=0}^{N-1} \beta_{j} \varphi_{j} \in E$; concluímos que $C^{\infty}\left(A_{\epsilon}\right)=S^{\circ} \oplus E$, já que a inclusão $S^{\circ} \oplus E \subset C^{\infty}\left(A_{\epsilon}\right)$ é direta.

Como $E$ tem dimensão $N, \mathcal{F}=S^{\circ}$ tem codimensão igual a $N$; portanto finita.

Com os argumentos anteriores também provamos que $\mathcal{F}$ tem codimensão igual a $r$ quando é definido em (3).

Finalmente, suponha que $\mathcal{F}$ definido em (4).

Defina

$$
q \doteq p-p_{0}-\ell_{0} x^{r-1}\left(x^{n-r} a_{0}+i x^{m-r} b_{0}\right)
$$

Sob nossas hipóteses, obtemos

$$
\int_{0}^{2 \pi} \frac{\partial^{j} q}{\partial x^{j}}(0, t) d t=0, j=0, \ldots, r-1 .
$$

Segue de (3.75) e do Teorema 1.8, que existe $\varphi \in C^{\infty}\left(A_{\epsilon}\right)$ tal que $L \varphi(x, t)=q(x, t)$. Note que, $\frac{\partial \varphi}{\partial t}(0, t)=0, \forall t \in S^{1}$. Portanto, temos $t \mapsto \varphi(0, t)$ constante. Então, podemos 
supor que $\varphi(0, t) \equiv 0$ (substituindo, se necessário, $\varphi(x, t)$ por $\varphi(x, t)-\varphi(0, t)$ ), e concluir que a condição adicional (3.14) descrita na demonstração da Proposição 3.3 pode ser escrita como

$$
\left\langle e^{-P_{0}-\varphi}\left(1 \otimes \delta^{\left(\ell_{0}+r-1\right)}\right), f\right\rangle-\sum_{\ell=r-1}^{\ell_{0}+r-2} B_{\ell}\left\langle e^{-P_{0}-\varphi}\left(1 \otimes \delta^{(\ell)}\right), f\right\rangle=0,
$$

sendo $B_{\ell}$ uma constante, para cada $\ell=r-1, \ldots, \ell_{0}+r-2$.

Defina $\mu_{\ell_{0}+r-1} \in \mathcal{E}^{\prime}\left(A_{\epsilon}\right)$ por

$$
\mu_{\ell_{0}+r-1} \doteq e^{-P_{0}-\varphi}\left(1 \otimes \delta^{\left(\ell_{0}+r-1\right)}\right)-\sum_{\ell=r-1}^{\ell_{0}+r-2} B_{\ell} e^{-P_{0}-\varphi}\left(1 \otimes \delta^{(\ell)}\right)
$$

Com os mesmos argumentos usados no caso em que $\mathcal{F}$ satisfaz a condição (2), concluímos que $\left.\mathcal{F}=S^{\circ} \cap\left\{f \in C^{\infty}\left(A_{\epsilon}\right) ;\left\langle\mu_{\ell_{0}+n-1}, f\right\rangle=0\right\rangle\right\}$, e que $\mathcal{F}$ tem codimensão igual a $r$.

\subsection{Resultados finais}

Nesta seção utilizaremos os resultados das seções anteriores para concluir sobre a resolubilidade de $\mathcal{L}_{p}$ em $\Sigma$, sendo $\mathcal{L}_{p}$ definido em (3.2) com $p \in C^{\infty}\left(A_{\epsilon}\right)$.

Pelo Lema 3.17, o resultado no Teorema 3.1 pode ser reescrito como:

Teorema 3.18. Se p satisfaz (3.7) e (3.8) então $\mathcal{L}_{p}$ é resolúvel em $\Sigma$.

Combinando as Proposições 3.3, 3.4 e 3.7 obtemos o seguinte resultado sobre a equação $\mathcal{L}_{p} u=f$.

Teorema 3.19. Assuma que (3.7) ou (3.8) falha. Defina o inteiro $j_{0}$ como segue:

(i) se $\Re\left(\lambda_{j}\right)=0$ para cada $j \in\{0, \cdots, r-1\}$, seja $j_{0}=r-1$;

(ii) se $\Re\left(\lambda_{j}\right) \neq 0$ para algum $j \in\{0, \cdots, r-1\}$, seja $j_{0}=\min _{0 \leqslant j \leqslant r-1}\left\{j: \Re\left(\lambda_{j}\right) \neq 0\right\}$.

Se $m-n \leqslant j_{0}$ então $\mathcal{L}_{p}$ é resolúvel em $\Sigma$.

Note que o resultado do Teorema 3.19 segue da Proposições 3.7 após utilizarmos as Proposições 3.3 e 3.4 para reduzir o estudo da resolubilidade da equação $\mathcal{L}_{p} u=f$, em uma vizinhança de $\Sigma$, ao estudo da resolubilidade da equação

$$
L u=\left(\sum_{j=0}^{r-1} \lambda_{j} x^{j}\right) u+f
$$


em uma vizinhança de $\Sigma$, com $f$ flat em $\Sigma$.

Finalmente, combinando as Proposições 3.13 e 3.16, finalizamos o estudo da equação $\mathcal{L}_{p} u=f$.

Teorema 3.20. Assuma que (3.7) ou (3.8) falha. Defina o inteiro $j_{0}$ como segue:

(i) se $\Re\left(\lambda_{j}\right)=0$ para cada $j \in\{0, \cdots, r-1\}$, seja $j_{0}=r-1$;

(ii) se $\Re\left(\lambda_{j}\right) \neq 0$ para algum $j \in\{0, \cdots, r-1\}$, seja $j_{0}=\min _{0 \leqslant j \leqslant r-1}\left\{j: \Re\left(\lambda_{j}\right) \neq 0\right\}$.

Suponha que $\Re\left(\lambda_{j_{0}}\right) a_{0}(0)<0$ ou que $\Re\left(\lambda_{j_{0}}\right) a_{0}(0)>0$ e $n-j_{0}$ é par. Se $m-n>j_{0}$ então $\mathcal{L}_{p}$ não é resolúvel em $\Sigma$.

Demonstração. Assuma que $\Re\left(\lambda_{j_{0}}\right) a_{0}(0)<0$ ou que $\Re\left(\lambda_{j_{0}}\right) a_{0}(0)>0$ e $n-j_{0}$ é par. Suponha, por absurdo, que existe um subespaço $\mathcal{F} \subset C^{\infty}\left(A_{\epsilon}\right)$ de codimensão finita tal que para cada $f \in \mathcal{F}$, existe $u \in C^{\infty}\left(A_{\epsilon}\right)$ solução da equação $\mathcal{L}_{p} u=f$ em uma vizinhança de $\Sigma$.

Sob nossas hipóteses existe $f \in C^{\infty}\left(A_{\epsilon}\right)$, flat em $\Sigma$, de forma que a equação $\mathcal{L}_{p} u=f$ não tem solução de classe $C^{\infty}$ em nenhuma vizinhança de $\Sigma$.

Usando série parcial de Fourier, escrevemos

$$
f(x, t)=\sum_{k \in \mathbb{Z}} \widehat{f}_{k}(x) e^{i k t}
$$

Para cada número primo $\ell$, defina

$$
F_{\ell}(x, t) \doteq \sum_{k \in \ell \mathbb{Z}} \widehat{f}_{k}(x) e^{i k t}
$$

Note que $B=\left\{F_{\ell}: \ell\right.$ é um número primo $\}$ é linearmente independente, já que para cada $\ell$ primo, o termo $\widehat{f}_{\ell} e^{i \ell t}$ comparece em $F_{\ell}$ mas não comparece em nenhum outro elemento de $B$.

Sob nossas hipóteses, existem $\varphi_{1}, \ldots, \varphi_{\nu} \in C^{\infty}\left(A_{\epsilon}\right)$, para algum $\nu \in \mathbb{Z}_{+}$, tal que

$$
C^{\infty}\left(A_{\epsilon}\right)=\mathcal{F} \oplus E
$$

sendo $E=\operatorname{span}\left\langle\varphi_{1}, \ldots, \varphi_{\nu}\right\rangle$

Para cada $\ell$ primo, escrevemos

$$
F_{\ell}=\psi_{\ell}+\sum_{j=1}^{\nu} \alpha_{\ell, j} \varphi_{j}
$$


sendo $\psi_{\ell} \in \mathcal{F}$, e $\alpha_{\ell, j}, j=1, \ldots, \nu$, constantes.

Logo, $F_{\ell}-\psi_{\ell}=\sum_{j=1}^{\nu} \alpha_{\ell, j} \varphi_{j} \in E$ para todo $\ell$ primo. Defina

$$
H \doteq \operatorname{span}\left\langle F_{\ell}-\psi_{\ell}\right\rangle, \ell \text { primo }
$$

Note que $\operatorname{dim} H \leqslant \operatorname{dim} E$. Portanto, existe um inteiro positivo $\gamma \leqslant \nu$ tal que

$$
H=\operatorname{span}\left\langle F_{\ell_{j}}-\psi_{\ell_{j}}\right\rangle, j=1, \ldots, \gamma
$$

Seja $\ell$ um número primo tal que $\ell>\ell_{j}, j=1, \ldots, \gamma$. Como $F_{\ell}-\psi_{\ell} \in H$, existem constantes $\beta_{1}, \ldots, \beta_{\nu}$ tais que

$$
F_{\ell}-\psi_{\ell}=\sum_{j=1}^{\nu} \beta_{\ell, j}\left(F_{\ell_{j}}-\psi_{\ell_{j}}\right) .
$$

Defina

$$
\psi=F_{\ell}-\sum_{j=1}^{\nu} \beta_{\ell, j} F_{\ell_{j}} .
$$

Note que

$$
\psi=\sum_{k \in \Lambda} \widehat{f}_{k}(x) e^{i k t}+\sum_{k \in \mathbb{Z} \backslash \Lambda} \widehat{\psi}_{k}(x) e^{i k t}
$$

sendo $\Lambda=\left\{k \in \mathbb{Z}: \ell\right.$ divide $|k|$ para cada $\ell$ primo $\left.\ell \neq \ell_{j}\right\} \cap\left\{k \in \mathbb{Z}: \ell_{j}\right.$ não divide $|k|, j=$ $1, \ldots, \gamma\}$.

Com os mesmos argumentos utilizados para mostrar que $L u=p u+f$ não tem solução em nenhuma vizinhança de $\Sigma$, podemos mostrar que $L v=p v+\psi$ não tem solução em nenhuma vizinhança de $\Sigma$.

Por (3.77), obtemos

$$
\psi=\psi_{\ell}+\sum_{j=1}^{\nu} \beta_{\ell, j} \psi_{\ell_{j}} \in \mathcal{F} .
$$

Concluímos assim, que $\psi \in \mathcal{F}$, e que a equação $\mathcal{L}_{p} v=\psi$ não tem solução $C^{\infty}$ em nenhuma vizinhança de $\Sigma$, gerando uma contradição. 


\section{Referências Bibliográficas}

[1] Bergamasco, A. P., Cordaro, P. D., and Petronilho, G. Global solvability for a class of complex vector fields on the two-torus. Comm. Partial Differential Equations, 29 (2004), 785-819.

[2] Bergamasco, A.P., and Dattori da Silva, P.L. Global solvability for a special class of vector fields on the torus, Contemp. Math. 400 (2006) 11-20.

[3] Bergamasco, A. P., Dattori da Silva, P. L., and Ebert, M. R. Gevrey solvability near the characteristic set for a class of planar complex vector fields of infinite type, J. Diferential Equations 246(4) (2009), 1673-1702.

[4] Bergamasco,A.P., and Meziani, A. Solvability Near the Characteristic Set for a Class of Planar Vector Fields of Infinite Type, Ann. Inst. Fourier (Grenoble) 55 (2005) 77-112.

[5] Dattori da Silva, P.L. Nonexistence of Global Solutions for a Class of Complex Vector Fields on Two-Torus, J. Math. Anal. Appl. 351 (2009), pp. 543-555.

[6] Dattori da Silva, P. L. $C^{k}$-Solvability Near the Characteristic Set for a Class of Planar Complex Vector Fields of Infinite Type, Ann. di Mat. Pura Appl. (4) 189, 3 (2010), 403-413.

[7] Hörmander, L. Pseudo-differential operators of principal type, Singularities in Boundary Value Problems. Proc. NATO Adv. Study Inst. (1980), 69-96, NATO Adv. Study Inst. Ser. C: Math. Phys. Sci., 65 (1981).

[8] Hörmander, L. The Analysis of Linear Partial Differential Operators I. SpringerVerlag, 1983. 
[9] Hörmander, L. The analysis of linear partial differential operators IV, SpringerVerlag, 1984.

[10] Meziani, A. Elliptic Planar Vector Fields with Degeneracies, Trans. Amer. Math. Soc. 357 (2005)

[11] Rudin, W. Functional Analysis, Tata McGraw-Hill Publishing Company Ltd., New Delhi, 1973.

[12] Sussmann, H.J. Orbits of families of vector fields and integrability of distributions, Trans. Amer. Math. Soc. 180 (1973) 171-188.

[13] Treves, F. Topological Vector Spaces, Distributions and Kernels, Academic Press, New York, 1967. 